\title{
MULTI-HAZARD RELIABILITY ASSESSMENT OF OFFSHORE WIND TURBINES
}

\author{
A Dissertation \\ by \\ MARYAM MARDFEKRI RASTEHKENARI
}

\author{
Submitted to the Office of Graduate Studies of \\ Texas A\&M University \\ in partial fulfillment of the requirements for the degree of \\ DOCTOR OF PHILOSOPHY
}

$\begin{array}{ll}\text { Approved by: } & \\ \text { Co-Chairs of Committee, } & \begin{array}{l}\text { Paolo Gardoni } \\ \text { John Niedzwecki }\end{array} \\ \text { Committee Members, } & \begin{array}{l}\text { Luciana Barroso } \\ \text { Ozden Ochoa } \\ \text { Loren D. Lutes }\end{array} \\ \text { Jead of Department, } & \text { John Niedzwecki }\end{array}$

December 2012

Major Subject: Civil Engineering

Copyright 2012 Maryam Mardfekri Rastehkenari 


\begin{abstract}
A probabilistic framework is developed to assess the structural reliability of offshore wind turbines. Probabilistic models are developed to predict the deformation, shear force and bending moment demands on the support structure of wind turbines. The proposed probabilistic models are developed starting from a commonly accepted deterministic model and by adding correction terms and model errors to capture respectively, the inherent bias and the uncertainty in developed models. A Bayesian approach is then used to assess the model parameters incorporating the information from virtual experiment data. The database of virtual experiments is generated using detailed three-dimensional finite element analyses of a suite of typical offshore wind turbines. The finite element analyses properly account for the nonlinear soil-structure interaction. Separate probabilistic demand models are developed for three operational/load conditions including: (1) operating under day-to-day wind and wave loading; (2) operating throughout earthquake in presence of day-to-day loads; and (3) parked under extreme wind speeds and earthquake ground motions. The proposed approach gives special attention to the treatment of both aleatory and epistemic uncertainties in predicting the demands on the support structure of wind turbines. The developed demand models are then used to assess the reliability of the support structure of wind turbines based on the proposed damage states for typical wind turbines and their corresponding performance levels. A multi-hazard fragility surface of a given wind turbine support structure as well as the seismic and wind hazards at a specific site location are incorporated into a probabilistic framework to estimate the annual probability of failure of the support
\end{abstract}


structure. Finally, a framework is proposed to investigate the performance of offshore wind turbines operating under day-to-day loads based on their availability for power production. To this end, probabilistic models are proposed to predict the mean and standard deviation of drift response of the tower. The results are used in a random vibration based framework to assess the fragility as the probability of exceeding certain drift thresholds given specific levels of wind speed. 


\section{DEDICATION}

To my husband, my parents and my sisters 


\section{ACKNOWLEDGEMENTS}

I would like to express my sincere gratitude to my advisor, Dr. Paolo Gardoni, who has offered visionary and insightful guidance to me over the past four years. I would like to wholeheartedly thank him for his support and patient discussions that definitely enhanced my academic and professional qualities. I would certainly like to extend my gratitude to Dr. Jose Roesset for his brilliant guidance and support, even after his retirement. I learned a lot from him not only in the field of soil-structure interaction but also in life itself and he will be my inspirational role model. He provided my financial support for three years for which I really appreciate him. I would like to thank the members of my dissertation committee, Dr. John Niedzwecki, Dr. Loren Lutes, Dr. Ozden Ochoa and Dr. Luciana Barroso for all their help and thoughtful comments.

I would like to thank my husband, Vahid Bisad, who was also my officemate during my $\mathrm{PhD}$ studies and helped me through technical discussions. I cannot express greatly enough my appreciation to my parents and my sisters, who, despite the geographical distance, provided me with their unconditional love and support during my PhD studies.

I wish to acknowledge the great atmosphere of Texas A\&M University that helped me to study and be productive in my research in a friendly environment. I am extremely thankful to my friends at Texas A\&M University and specifically my friends at Persian Student Association (PSA.) 


\section{TABLE OF CONTENTS}

Page

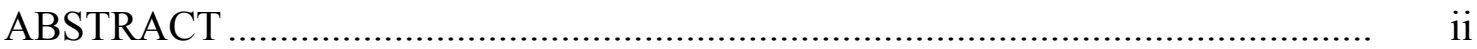

DEDICATION ..................................................................................... iv

ACKNOWLEDGEMENTS ........................................................................

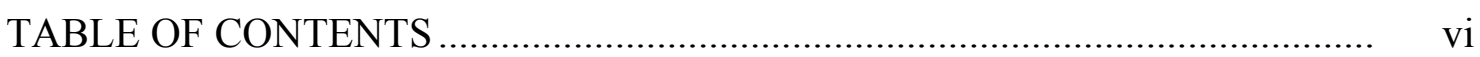

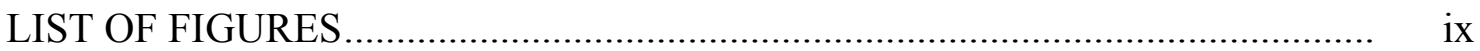

LIST OF TABLES ................................................................................

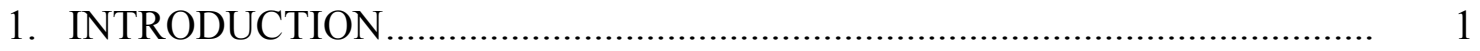

1.1 Problem statement .................................................................. 1

1.2 Background and technical needs ................................................ 3

1.3 Research objectives and methodology ....................................... 6

1.4 Organization of dissertation ......................................................... 8

2. MODELING LATERALLY LOADED SINGLE PILES ACCOUNTING FOR NONLINEAR SOIL-PILE INTERACTION .............................................. 12

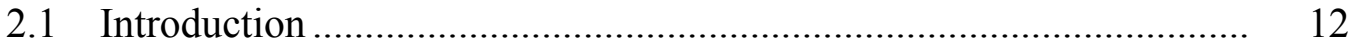

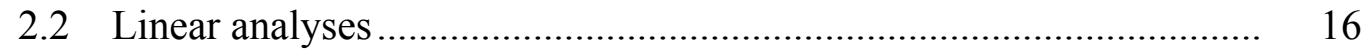

2.3 Nonlinear analyses ............................................................. 20

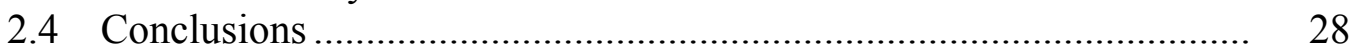

3. PROBABILISTIC DEMAND MODELS AND FRAGILITY ESTIMATES FOR OFFSHORE WIND TURBINES SUBJECT TO DAY-TO-DAY LOADS ...... 31

3.1 Introduction ......................................................................... 31

3.2 Virtual experimental data ........................................................ 33

3.2.1 Experimental design ...................................................... 33

3.2.2 Analytical modeling........................................................... 34

3.2.2 Equality and lower bound data .......................................... 40 
3.3 Probabilistic demand models ............................................................. 41

3.3.1 Deterministic demand model ................................................... 43

3.3.2 Model correction................................................................. 43

3.3.3 Model selection.................................................................. 45

3.3.4 Bayesian updating................................................................ 46

3.3.5 Probabilistic demand models .................................................... 47

3.4 Fragility estimates of an example offshore wind turbine support

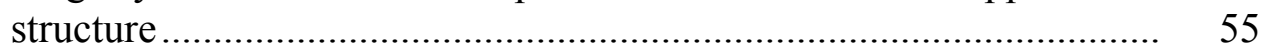

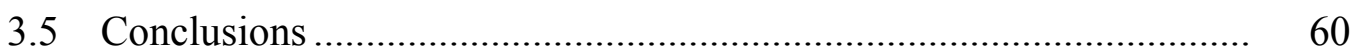

\section{PROBABILISTIC SEISMIC DEMAND MODELS AND FRAGILITY} ESTIMATES FOR OFFSHORE WIND TURBINES …………………......... 62

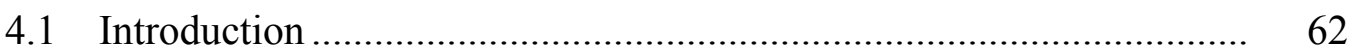

4.2 Probabilistic demand models ............................................................ 64

4.2.1 Deterministic demand models ................................................. 64

4.2.2 Model correction.................................................................. 67

4.2.3 Virtual experiment data …………………………................. 69

4.2.4 Model selection....................................................................... 71

4.2.5 Proposed probabilistic seismic shear and moment demand

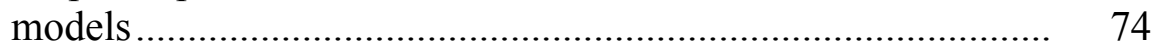

4.3 Seismic fragility estimates for an example offshore wind turbine

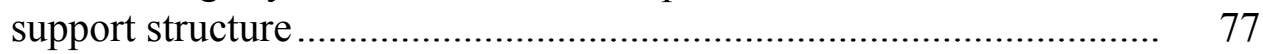

4.4 Conclusions ................................................................................. 81

\section{MULTI-HAZARD RELIABILITY ASSESSMENT OF OFFSHORE WIND}

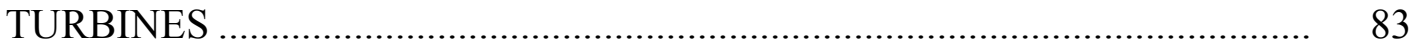

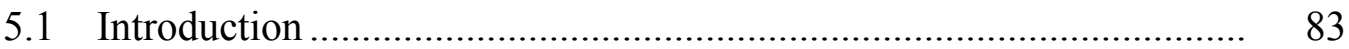

5.2 Multi-hazard assessment ............................................................... $\quad 85$

5.2.1 Seismic contribution to probability of failure............................ 86

5.2.2 Wind contribution to probability of failure................................ 87

5.3 Probabilistic demand models ............................................................ 88

5.3.1 Additional virtual experiment data .......................................... 89

5.3.2 Updated model .................................................................... 91

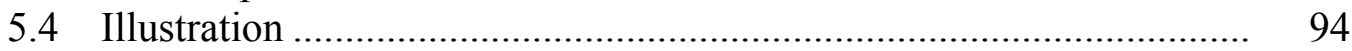

5.4.1 Predictive fragility ………………………………................ 95

5.4.2 Sensitivity measures ................................................................ 97

5.4.3 Importance measures .............................................................. 98

5.4.4 Annual probability of failure ................................................ 100

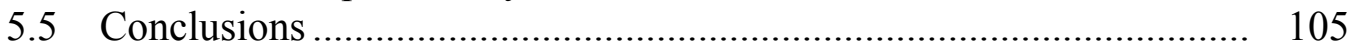


6. PROBABILISTIC ASSESSMENT OF EXCESSIVE DRIFTS FOR OFFSHORE WIND TURBINES .......................................................... 107

6.1 Introduction ........................................................................ 107

6.2 Dynamic response of offshore wind turbines.................................. 111

6.3 Development of the model .......................................................... 112

6.3.1 Model selection............................................................... 116

6.3.2 Model accuracy.......................................................... 121

6.4 Verification........................................................................... 123

6.5 Summary and conclusions....................................................... 126

7. CONCLUSIONS AND FUTURE WORK …............................................. 128

7.1 Conclusions ......................................................................... 128

7.2 Unique contribution................................................................ 130

7.3 Future work ................................................................................ 131

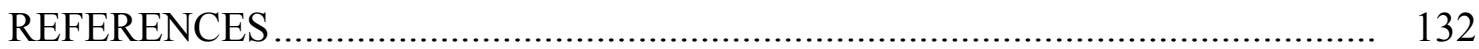




\section{LIST OF FIGURES}

Page

Figure 2-1 3D finite element model of the pile foundation ................................ 16

Figure 2-2 Deformation of the soil with the 3D model of the pile ...................... 18

Figure 2-3 Deformation of the soil with the 1D model of the pile ..................... 18

Figure 2-4 Variation of pile head displacement versus pile radius in linear analyses with constant $E_{p} I_{p}$ for the pile

Figure 2-5 Deformed mesh of the pile foundation in 3D nonlinear analysis

Figure 2-6 Variation of pile head displacement versus pile radius in nonlinear analyses for (a) sand and (b) clay with constant $E_{p} I_{p}$ for the pile ......

Figure 3-1 Finite element model of a sample wind turbine support structure in ABAQUS

Figure 3-2 Schematic illustration of wave relative to the mean sea level (MSL)..

Figure 3-3 Typical offshore wind turbine support structure configuration

Figure 3-4 Stepwise deletion process for deformation demand model, where $(\times)$ indicates term to be removed

Figure 3-5 Comparison between measured versus predicted deformation demands, (a) deterministic model, (b) median probabilistic model

Figure 3-6 Stepwise deletion process for shear demand model, where $(\times)$ indicates term to be removed

Figure 3-7 Comparison between measured versus predicted shear demands,

Figure 3-8 Stepwise deletion process for moment demand model, where $(\times)$ indicates term to be removed

Figure 3-9 Comparison between measured versus predicted moment demands, (a) deterministic model, (b) median probabilistic model 
Figure 3-10 Fragility estimates for an offshore wind turbine as a function of mean wind speed for $H_{s}=1 \mathrm{~m}$

Figure 3-11 Yield bending fragility estimates for an offshore wind turbine as a function of significant wave height at different wind speeds

Figure 4-1 Stepwise deletion process for seismic shear demand model, where $(\times)$ indicates term to be removed

Figure 4-2 Stepwise deletion process for seismic moment demand model, where $(\times)$ indicates term to be removed

Figure 4-3 Comparison between measured versus predicted seismic shear demands, (a) deterministic model, (b) median probabilistic model ....

Figure 4-4 Comparison between measured versus predicted seismic moment demands, (a) deterministic model, (b) median probabilistic model ....

Figure 4-5 Fragility estimates for a typical 5-MW offshore wind turbine as a function of spectral acceleration for both the yield and ultimate limit states

Figure 4-6 Fragility estimates for a typical 5-MW offshore wind turbine as a function of mean wind speed due to ultimate limit state.

Figure 5-1 Measured vs. predicted shear demands based on (a) deterministic and (b) probabilistic models

Figure 5-2 Measured vs. predicted moment demands based on (a) deterministic and (b) probabilistic models

Figure 5-3 Fragility estimates for a typical 5-MW offshore wind turbine as a function of (a) spectral acceleration and (b) mean wind speed.

Figure 5-4 Comparison between predictive fragility estimate by Monte Carlo simulations (solid lines) and FORM with reduced number of random variables (dotted lines)

Figure 5-5 Annual probability density function for spectral acceleration at Site I (dotted line) and Site II (solid line) 
Figure 5-6 Annual probability density function for wind speeds at (a) Site I and (b) Site II

Figure 6-1 Diagnostic plots for normality assumption along with the p-values of Kolmogorov-Smirnov test (KS P-value) for the drift responses of 9 wind turbine configurations considered in experimental design........

Figure 6-2 Comparison between the predictions of Eq. (6-5) and corresponding measured values for different drift thresholds

Figure 6-3 Values of the selection criteria versus number of explanatory functions for the mean model

Figure 6-4 Values of the selection criteria versus number of explanatory functions for the standard deviation model

Figure 6-5 Measured versus predicted mean of the drifts based on FAST models (left) and proposed models (right)

Figure 6-6 Measured versus predicted standard deviation of the drifts based on FAST models (left) and proposed models (right)

Figure 6-7 Predicted versus measured values of $v$ based on FAST models for drift thresholds of (a) $b=0.1 \%$, (b) $b=0.25 \%$, (c) $b=0.5 \%$ and (d) $\mathrm{b}=1 \%$

Figure 6-8 Predicted versus measured values of $v$ based on proposed models for drift thresholds of (a) $b=0.1 \%$, (b) $b=0.25 \%$, (c) $b=0.5 \%$ and (d) $\mathrm{b}=1 \%$

Figure 6-9 Schematic 3D curve for the probability of exceeding drift thresholds versus threshold values and wind speed for the example wind turbine

Figure 6-10 Probability of exceeding drift thresholds versus wind speed for the example wind turbine

Figure 6-11 Probability of exceeding drift thresholds versus threshold values for the example wind turbine at (a) rated wind speed and (b) cut-out wind speed 


\section{LIST OF TABLES}

Page

Table 2-1 Properties of the pile

Table 2-2 Variation of pile head displacement versus pile radius in different linear analysis methods with constant $E_{p} I_{p}$ for the pile

Table 2-3 Elastic-plastic properties of soil.

Table 2-4 Variation of pile head displacement versus pile radius in different Nonlinear analysis methods with constant $E_{p} I_{p}$ for the pile.

Table 3-1 Geometrical and mechanical properties used in experimental design...

Table 3-2 Explanatory functions for demand models

Table 3-3 Posterior statistics of the parameters in the deformation demand model

Table 3-4 Posterior statistics of the parameters in the shear demand model

Table 3-5 Posterior statistics of the parameters in the moment demand model.....

Table 3-6 Proposed damage states and corresponding limit states and natural hazards

Table 3-7 Properties of the NREL offshore 5-MW baseline wind turbine

Table 4-1 Explanatory functions for demand models

Table 4-2 Bins from which ground motions are selected.

Table 4-3 Posterior statistics of the parameters in the shear demand model

Table 4-4 Posterior statistics of the parameters in the moment demand model.....

Table 4-5 Updated damage states and the corresponding performance levels during earthquake......

Table 5-1 Posterior statistics of the parameters in the shear demand model 
Table 5-2 Posterior statistics of the parameters in the moment demand model.... 94

Table 5-3 Distribution, mean, and COV for random variables in the limit state function.

Table 5-4 Sensitivity measures for ultimate and yield bending moment failure modes

Table 5-5 Importance measures for ultimate and yield bending moment failure modes

Table 5-6 BIW distribution parameters...................................................... 103

Table 5-7 Annual probabilities of failure ............................................... 105

Table 6-1 Candidate explanatory functions for the developed models for mean and standard deviation

Table 6-2 Statistical properties of model parameters

Table 6-3 RSME values for the predictions of $v$

Table 6-4 Properties of the example wind turbine considered for the verification of the developed models 


\section{INTRODUCTION}

\subsection{Problem Statement}

Wind energy has been used for more than 2,500 years. The first windmills, built in 644 A.D. in the Persian-Afghan border region of Sistan, were used for milling grains (Hau, 2006.) Over the past decades, continually growing energy demands as well as climate change and other pollution problems have resulted in a considerable anticipation about wind energy seen as one of the most attractive and promising renewable sources of energy. According to the annual reports by the Global Wind Energy Council (Sawyer and Rave, 2012), the global cumulative installed wind capacity has been doubling every three years and it is projected to continue to grow at a similar rate.

Wind turbines are separated into two types based on the axis about which the turbine rotates: horizontal axis wind turbines (HAWT) and vertical axis wind turbines (VAWT.) The main advantage of VAWTs is that the operation is independent of the wind direction. Also the machinery is usually located at the tower base and is easily accessible for maintenance. But, large VAWTs are usually associated with stability and dynamic problems, and also most VAWTs produce energy with less efficiency than HAWTs because of the additional drag that they have as their blades rotate into the wind (Fink, 2005.) Therefore the horizontal axis turbines are more commonly used as large electricity generator wind turbines.

Wind turbines may be installed onshore or offshore. Onshore and offshore wind turbines differ in design and construction because of the differences in the environmental 
conditions and loadings. They also differ in the support structure and foundation type. Gravity-based foundations, rock anchors, prestressed concrete cylinders and pile groups are the most common foundation types for onshore wind turbines. For offshore wind turbines gravity-based foundations, steel pipe mono-piles and tripods are commonly used, depending on the sea depth (Bonnett, 2005.) Moreover, floating foundation systems are the typical choice for offshore wind turbines installed in deep waters beyond $50 \mathrm{~m}$ (Musial and Butterfield 2004.)

The focus of this study is on the reliability assessment of modern horizontal axis offshore wind turbines installed in water depths less than 30 meters, supported by monopile foundations.

Furthermore, offshore wind turbines installed extensively around the world are subject to different hazards (e.g., earthquake, hurricane, and typhoon) raising concerns about the reliability of the wind turbine support structure. For instance, Japan is the world's $13^{\text {th }}$ largest producer of wind power according to the World Wind Energy Association (Gsänger and Pitteloud, 2012), despite having a considerably high occurrence rate of earthquakes and typhoons. Likewise, according to the National Renewable Energy Laboratory (Flowers, 2012), California, a highly seismic region, is the third largest wind power producer in the nation. Moreover, the wind industry is recently considering installing offshore wind farms in the south coast of the United States, and in particular in the Gulf of Mexico, because of the superior wind resources available in this region (Schwartz et al. 2010.) However, a considerably high hurricane occurrence rate in the Gulf of Mexico raises a new concern about the safety of wind 
turbine support structures subject to hurricane. To investigate the reliability of a wind turbine support structure, all possible hazards that can occur during the wind turbine's life have to be considered. To this end, a probabilistic framework is needed to evaluate the safety of the support structure under multiple hazards and predict its annual probability of failure. The results can assist the wind industry decision makers in choosing optimum design and location for future wind energy projects.

Moreover, the cost of energy is a key to evaluate the success of an energy project. The cost of energy in a wind energy project is the total cost of the wind farm including the cost of manufacturing and installation, and also operation and maintenance costs. Reliability analysis can help ensure the success of an energy project. Providing adequate reliability can help reduce the need for costly repairs and downtime. At the same time, knowledge of the reliability level of a design can be used to avoid wastefully overdesigning a wind turbine. In general, a reliability-based design of wind turbines would allow for the optimal allocation of resources for energy production. To this end, it is of interest to forecast the performance of wind turbines in terms of their unavailability for power production. As part of this study, wind turbines unavailability is investigated based on exceeding certain drift thresholds.

\subsection{Background and Technical Needs}

Several probabilistic studies have been conducted on wind turbines. Walford (2006) and Tavner et al. (2007) investigated the reliability of operation and power production of wind turbines based on historical data of failures and their associated costs. Walford 
(2006) also discussed the means for reducing operation and maintenance costs. However, a methodology that relies on the structural response of wind turbines will provide a more accurate estimation of their reliability. Madsen et al. (1999), Agarwal and Manuel (2008), and Manuel et al. (2001) employed probabilistic frameworks to predict the extreme and fatigue loads for the design of onshore and offshore wind turbines based on the dynamic response of the support structures. Although the aeroelastic interaction is successfully considered in the analyses, these studies fail to incorporate the foundation stiffness in the dynamic response of wind turbines.

Offshore wind turbines installed in water depths less than 30 meters are typically supported by mono-pile foundations. Bush and Manuel (2009) investigated the effect that the use of alternative models for mono-pile foundation of shallow-water offshore wind turbines has on the design extreme loads. Their results showed the importance of incorporating foundation stiffness in the simulations.

Aeroelastic simulators such as FAST (Jonkman and Buhl Jr. 2005), ADAMS (Laino and Hansen 2001), and GH Bladed (Bossanyi 2000) successfully include the aeroelastic interactions in the analysis of dynamic response of the support structure. However, an important limitation of these simulators is that they are not capable of continuous modeling of the nonlinear foundation system and the dynamic soil-structure interaction. A finite element (FE) analysis of the support structure and the foundation can be done to account for the nonlinear foundation behavior and the dynamic soilstructure interaction. However, a detailed nonlinear FE analysis can be quite expensive and time consuming both in developing and running it. In addition, assessing the 
reliability of a wind turbine requires accounting for the uncertainties inherent in the structural material, soil and geometrical properties. To account for such uncertainties, a high number of FE analyses would need to be carried out making this approach too time consuming.

To address the concern related to the installation of wind farms in moderate and high seismic regions, a number of researchers conducted studies on the seismic response of wind turbines. Early publications on the analysis of dynamic response of wind turbines during earthquake (Bazeos et al. 2002, Lavassas et al. 2003) were based on the simplified models that lumped the nacelle and rotor as a point mass at the top of the tower. As a result the aeroelastic interaction was not accounted for. More recently, Witcher (2005) and Prowell et al. (2009) developed more refined models that considered the aeroelastic interaction. Specifically, Witcher (2005) studied the seismic response of support structures for both operating and parked wind turbines. The results showed the importance of accounting for aeroelastic interaction for operating wind turbines. Prowell et al. (2009) calibrated the aeroelastic interaction modeled in FAST using experimental data from a shake-table test of a small onshore 65-kW wind turbine (Prowell et al. 2008.) Yet, both studies fail to incorporate the dynamic soil-structure interaction.

With the limitations in the current practice of the structural analysis of wind turbines, it is of interest to investigate the performance of offshore wind turbines under multiple hazards incorporating the uncertainties inherent in the structural material, soil and geometrical properties as well as the influence of soil-structure interaction on dynamic response of the support structure. 


\subsection{Research Objectives and Methodology}

This study addresses the following three research objectives: (1) assessment of the demands on the support structure of offshore wind turbines accounting for the inherent uncertainties as well as dynamic soil-structure interaction; (2) multi-hazard assessment of the structural reliability of offshore wind turbines; and (3) evaluation of the performance of wind turbines in terms of their availability for power production.

To address the stated objectives, this study generates a database of virtual experiments by conducting detailed three-dimensional (3D) nonlinear FE analyses of the dynamic response of the support structures of a suite of typical offshore wind turbines supported by mono-piles. The FE models included a continuous modeling of the pile and the surrounding soil. As a result, the FE models successfully incorporated the dynamic soil-structure interaction into the response of the support structure. The virtual experiment database is then used to calibrate simplified probabilistic models for the deformation, shear force and bending moment demands on the support structure under day-to-day loading in operating conditions (i.e., day-to-day wind, wave and current loads.) The developed probabilistic models provide unbiased predictions for the deformation, shear and moment demands on the support structures, accounting for the inherent uncertainties, including the statistical uncertainty (associated with the finite sample size) and the modeling errors (associated with the selection of the variables in the models and the model forms.) The proposed approach gives special attention to the treatment of both aleatory and epistemic uncertainties in predicting the demands on the support structure of wind turbines. Aleatory uncertainty (or randomness) is inherent in 
the nature and is irreducible. Epistemic uncertainty arises from our lack of knowledge, errors in measuring observations and finite size of observation samples, and is reducible. The former is present in the structural and loading variables and the latter is present in the model parameters. The proposed demand models are then used to assess the structural reliability of a typical 5-MW offshore wind turbine for different performance levels proposed for operating wind turbines.

To address the concerns related to safety of wind turbines subject to earthquake, the next step is to use developed FE models to conduct time-history analyses of offshore wind turbines subject to seismic loading in addition to day-to-day operational loading accounting for the dynamic soil-structure interaction. Using the generated data, novel probabilistic models are developed for the seismic demands on the support structures. The developed probabilistic models were then used to assess the reliability of the support structures conditioning on spectral acceleration and the mean wind speed acting on the structure.

Furthermore with the new concern about the safety of wind turbines in regions prone to hurricane, proposed seismic demand models are updated incorporating the information from additional virtual experiment data. Additional virtual experiment data are generated from the dynamic analyses of the developed 3D nonlinear FE models subject to extreme wind speeds during hurricane in addition to earthquake ground motions. Developed probabilistic models are then used to assess the conditional failure probability (fragility) of the support structure for an example offshore wind turbine for given intensity measures of the seismic and wind loading. The multi-hazard fragility 
surface of the given wind turbine support structure as well as the seismic and wind hazards at a specific site location are incorporated into the probabilistic framework to estimate the annual probability of failure of the support structure.

Finally, the serviceability of wind turbine support structures is explored in terms of wind turbines availability for power production. A framework is proposed to investigate the unavailability of offshore wind turbines based on exceeding certain drift thresholds. The probability and expected time of exceeding specific drift thresholds are estimated based on the mean and standard deviation of the drift response. To this end, probabilistic models are proposed to predict the mean and standard deviation of the drift response of the tower, based on the information obtained from virtual experiment database generated in this study.

\subsection{Organization of Dissertation}

This dissertation is organized using a section-subsection format. The following six sections discuss the details of the methodology developed in this study to address the stated research objectives in the previous subsection. Following is a brief overview of each section in this dissertation.

- Section 1 (current section) provides an introduction about the problem, including problem statement, background and technical needs, research objectives and methodology, and organization of dissertation.

- Section 2 investigates the behavior of a laterally loaded mono-pile foundation using the finite element method (FEM) to account for soil-pile interactions. Prevailing 
simple methods for predicting the deflection of laterally loaded single piles in sand and clay are evaluated using linear and nonlinear finite element analyses. FE analyses are conducted using continuous 3D modeling of the pile and surrounding soil accounting for the pile-soil interaction. The results in Section 2 indicate the importance of continuous modeling of mono-pile foundations rather than using simplified methods such as $p-y$ method and in particular, one dimensional beamcolumn elements in order to account for the nonlinear soil-structure interaction, particularly for the pile sizes typical of foundations of offshore wind turbines.

- Section 3 develops probabilistic models to predict the deformation, shear and moment demands on the support structure of wind turbines operating under day-today wind, wave and current loads. An existing deterministic model is corrected by adding a correction term to capture the inherent bias, and model error arising from an inaccurate model form or missing variables. A database of structural responses is used to calibrate the proposed models. The database is obtained from detailed 3D nonlinear FE analyses of a set of typical wind turbine systems with different design parameters. The finite element analyses account for the nonlinear soil-structure interaction. The proposed probabilistic demand models provide accurate and unbiased estimates of the demands on the support structure properly accounting for the underlying uncertainties. The models are then used to estimate the fragility of the support structure of wind turbines which is defined as the conditional probability of not meeting specified capacity levels. 
- Section 4 develops probabilistic models for seismic shear and moment demands on the support structure of wind turbines operating throughout earthquake in presence of day-to-day loading, following an approach consistent with the one used in Section 3. Developed seismic demand models are then used to assess the fragility of the support structure of a typical 5-MW wind turbine for given intensity measures of spectral acceleration and mean wind speed.

- Section 5 develops a probabilistic framework to assess the structural reliability of offshore wind turbines under multiple hazards. A multi-hazard fragility surface of a given wind turbine support structure as well as the seismic and wind hazards at a specific site location are incorporated into the probabilistic framework to estimate the annual probability of failure of the support structure. The seismic demand models developed in Section 4 are updated incorporating the information obtained from additional experiment data generated for wind turbines subject to extreme wind speeds during hurricane and earthquake ground motions. Updated probabilistic demand models are then used to estimate the fragility of the support structure of a given wind turbine. As an example of the proposed framework, the annual probability of failure is calculated for two identical wind turbines, one located in the Gulf of Mexico of the Texas Coast (prone to hurricanes) and one off the California Coast (a high seismic region.)

- Section 6 proposes a framework to explore the performance of offshore wind turbines based on exceeding certain drift thresholds. For this purpose, novel models are developed to predict the mean and standard deviation of drift response of wind 
turbine support structures operating under day-to-day loads. The developed models are then used in a random vibration based framework to estimate the probability and expected time of exceeding drift thresholds. The virtual experiment database generated earlier in Section 3 is used to assess the parameters of the developed models. To verify the developed models, the probability of exceeding specific drift thresholds are estimated for a typical offshore wind turbine based on both simulations conducted using commonly used wind turbine simulators and proposed model, and the results are compared to the accurate estimations based on detailed 3D nonlinear FE analyses.

- Section 7 provides the conclusion of this dissertation along with the unique contribution of this work as well as suggestions for the future work. 


\section{MODELING LATERALLY LOADED SINGLE PILES ACCOUNTING FOR NONLINEAR SOIL-PILE INTERACTION}

\subsection{Introduction}

Pile foundations are widely used to support laterally loaded structures especially offshore. The extensive growth of wind farms around the world has raised new concerns about the accuracy of the analysis and design methods for laterally-loaded largediameter mono-piles (the most popular foundation structure for offshore wind turbines.)

Common methods for the analysis of laterally loaded single piles can be generally classified into two categories: (1) Winkler (elastic) foundation models and (2) continuous models accounting for the coupling of forces and displacements in the soil along the pile. In each category the analysis may be static (monotonic or cyclic loading) or dynamic. Also the behavior of the soil, pile and soil-pile interaction may be considered as linear or nonlinear.

Winkler foundation models are popular because of their simplicity and reasonable accuracy. When the elastic stiffness of the foundation can be considered constant with depth one can even obtain simple closed form solutions for the pile head stiffness and flexibility (Sanchez Salinero 1982.) The main difference between the different Winkler foundation models available is in the selection of the foundation stiffness coefficients. For dynamic problems Novak (1975) has proposed the use of Winkler foundation coefficients based on Baranov's equations (1967) for in plane and out of plane vibrations of a disk. The corresponding horizontal $k_{x}$ and rotational $k_{\varphi}$ 
springs per unit of length along the pile are functions of a dimensionless frequency $a_{0}=\omega_{0} r_{p} / C_{s}$ where $\omega_{0}=$ the frequency in radians/second, $r_{p}=$ the radius of the pile, and $C_{s}=$ the shear wave velocity of the soil. Unfortunately the horizontal term tends to zero at a zero frequency representing the static case. As a result it is common to use the values corresponding to a dimensionless frequency of 0.3 for smaller frequencies (Sanchez Salinero 1982.) In that case, $k_{x}=4 G_{\text {soil }}$ and $k_{\varphi}=2.6666 G_{\text {soil }} r_{p}{ }^{2}$, where $G_{\text {soil }}=$ the shear modulus of the soil.

For nonlinear analyses the $p-y$ method is the most commonly used in this category. It employs an elastic beam column member to model the pile and nonlinear horizontal springs to represent the soil reactions. The $p-y$ curves describe the nonlinear behavior of the soil springs. They were developed first by Matlock (1970) for soft clays under the water table. Reese and Welch (1975) and Reese et al. (1975) developed $p-y$ curves for hard clays subjected to monotonic and cyclic loading, above and under the water table respectively. Analyzing the results of the full scale tests conducted by Reese et al. (1975), Dewaikar et al. (2009) presented a modified approach to construct $p-y$ curves in stiff clay. In another study Kim and Jeong (2011) developed a framework based on 3D finite element analysis for determining a $p-y$ curve. The $p-y$ curves for sands were also developed by Reese et al. (1974) for monotonic and cyclic loading. Briaud et al. (1985) developed an alternative method to obtain the $p-y$ curves directly from pressuremeter tests. The method was reasonably accurate but complicated and time consuming, so Briaud (1997) developed a simpler approach called "simple approach for 
lateral loads on piles" or SALLOP, using the pressuremeter limit pressure and the pressuremeter modulus.

A number of recent studies have been conducted to predict the behavior of laterally loaded piles in different soil conditions. Sanjaya Kumar et al. (2007) used ABAQUS (2007) and the $p-y$ method to study the behavior of laterally loaded pile foundations in high marine clay. Suleiman et al. (2010) conducted a test to measure the soil-pile interaction pressure for small diameter piles in loose sand that the results can be used in developing the soil force-displacement relationship (i.e. the soil reaction or the $p$ $y$ curve.) An equivalent model for a laterally loaded linear pile-soil system was presented by Chioui and Chenu (2007) using artificial lateral springs.

Continuous modeling of the pile and the surrounding soil are mostly done using finite element or boundary element models. Both methods can provide rigorous solutions accounting for soil-pile interaction under static and dynamic loading. For the linear case an accurate solution was proposed by Blaney et al. (1976) using the consistent boundary matrix developed by Kausel (1974) to reproduce the soil cavity occupied by the pile and adding then the pile enforcing compatibility of horizontal and vertical displacements between pile and soil along the pile. An extensive number of studies were carried out by Sanchez Salinero (1982) comparing the results of this approach to those provided by a variety of other methods and proposing approximate formulas for the pile head stiffness. This approach is only valid however in the linear elastic range. The finite element method is particularly convenient when desiring to account for nonlinear effects including the nonlinear behavior of the soil and of the soil-pile interface. 
A 3D nonlinear finite element analysis of a pile foundation in which both the soil and the pile are modeled with 3D finite elements can be quite expensive and time consuming, particularly when incorporating nonlinear behavior. As a result some investigators have used finite element models that represent the pile by an elastic beamcolumn member without transverse dimensions (only the centroidal axis) and only the soil with $3 \mathrm{D}$ solid elements. This method takes into account the continuity of the soil mass and is easy to use for linear static and dynamic analysis. However, the most important limitation of this approach is that it does not take into account the dimension of the pile section.

This section evaluates prevailing approaches for modeling linear and nonlinear behavior of pile foundations. As a first step the models used for the analyses of pile foundations are validated. Then the model selected is implemented in the computer program ABAQUS using 3D brick elements to discretize the soil around the pile and shell elements to model the hollow pile. The results obtained with this model for linear and nonlinear analyses are compared to those provided by a variety of other methods used in practice.

In the following, four different models used for linear analysis of single pile foundations are examined and the influence of accounting for the pile diameter in the simplified linear FE analyses is evaluated. In the next subsection, the 3D finite element model is improved by accounting for the nonlinearity of the soil and soil-pile interaction. Two common simplified nonlinear models are then evaluated using this model for mono-piles in sand and clay. 
Table 2-1. Properties of the pile

\begin{tabular}{lcc}
\hline Parameter & Symbol & Value \\
\hline Penetration depth $(\mathrm{m})$ & $H_{p}$ & 21.0 \\
Radius $(\mathrm{m})$ & $r_{p}$ & 2.00 \\
Wall thickness $(\mathrm{m})$ & $t_{p}$ & 0.05 \\
Modulus of elasticity $(\mathrm{kPa})$ & $E_{p}$ & $2.0 \mathrm{E} 8$ \\
Unit weight $\left(\mathrm{kN} / \mathrm{m}^{3}\right)$ & $\gamma_{p}$ & 87.00 \\
Poisson ratio & $v_{p}$ & 0.30 \\
\hline
\end{tabular}

\subsection{Linear Analyses}

Analyses considering linear soil behavior and perfect bonding between the pile and the surrounding soil are conducted first. The pile selected for the study is hollow with a diameter of $4 \mathrm{~m}$ and the properties listed in Table 2-1. Four different models are studied:

1) The first model is a $3 \mathrm{D}$ finite element model of both the soil around the cavity occupied by the pile (solid elements) and for the pile, with shell elements for hollow piles and brick elements for solid piles (shown in Figure 2-1)

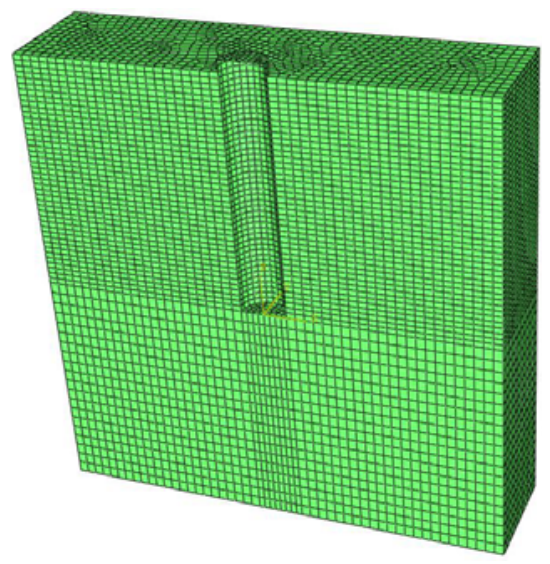

Figure 2-1. 3D finite element model of the pile foundation 
2) The second simpler model reproduces the soil with solid elements filling the space without any cavity. The pile is represented by the centroidal axis of a one dimensional (1D) beam column coinciding with the central axis of the soil model, enforcing only compatibility of horizontal displacements between the nodes of the pile and those of the soil along the axis.

3) The third model is the one proposed by Blaney et al. (1976) with the consistent boundary matrix with the radius of the cavity representing the soil and enforcing compatibility of both horizontal and vertical displacements between the soil and the pile along its sides.

4) The fourth model is a beam on an elastic (Winkler) foundation with horizontal and rotational springs along the side of the pile. The constants selected for the foundation are $k_{x}=4 G_{\text {soil }}$ and $k_{\varphi}=2.6666 G_{\text {soil }} r_{p}^{2}$.

The pile is subjected at the head to a vertical load of 5,000 kN, a horizontal load of 2,503 kN, and a moment of $84,983 \mathrm{kNm}$. These are values obtained considering the extreme forces on an example offshore wind turbine. For the linear analyses the soil is assumed to have a Young's modulus $E_{\text {soil }}=50,000 \mathrm{kPa}$, a Poisson's ratio $v_{\text {soil }}=0.3$, and a unit weight $\gamma_{\text {soil }}=20 \mathrm{kN} / \mathrm{m}^{3}$.

The predicted deflections at the pile head by the four models are $20.9 \mathrm{~mm}$ for the 3D FE pile model, $68.3 \mathrm{~mm}$ for the 1D FE pile model, $20.5 \mathrm{~mm}$ for the consistent boundary matrix and $24.3 \mathrm{~mm}$ for the Winkler foundation. The deformation of the soil with the 3D finite element model is shown in Figure 2-2 while Figure 2-3 shows the corresponding deformations with the 1D model of the pile. The results, obtained using 
the 3D finite element model are in good agreement with the approach that employs consistent boundary matrix (less than $2 \%$ off.) The agreement with the results of the Winkler foundation is not quite as good but still acceptable (about $20 \%$ off.) The model without the cavity and with the pile as a 1D linear element yields deflections that are $200 \%$ too large.

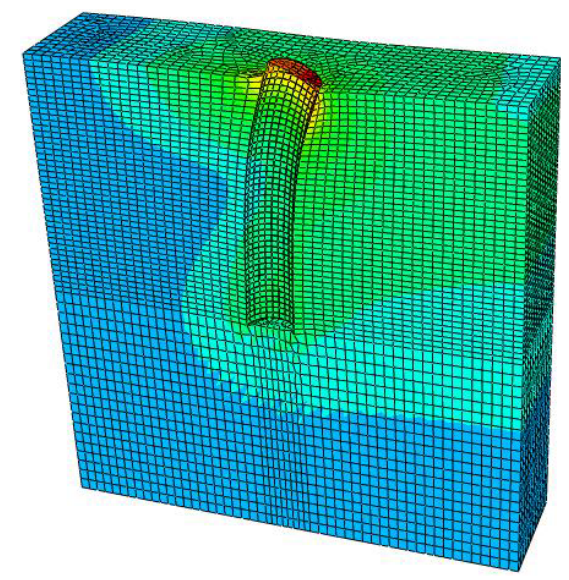

Figure 2-2. Deformation of the soil with the 3D model of the pile

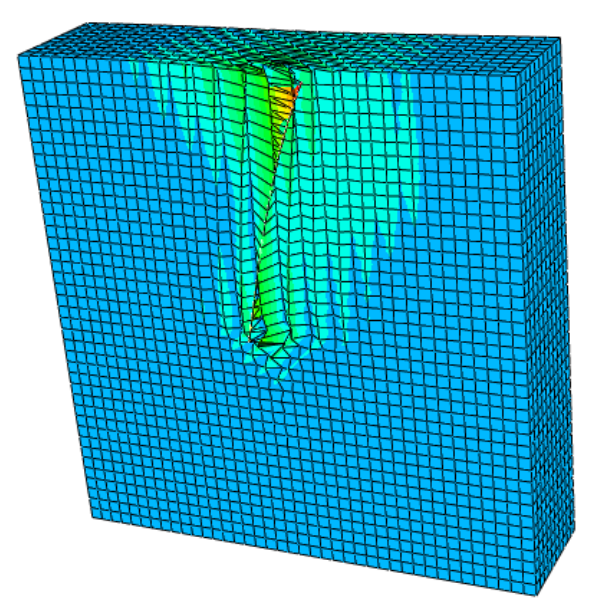

Figure 2-3. Deformation of the soil with the 1D model of the pile 
To understand better the reasons for this large discrepancy it was decided to conduct studies for other pile sizes. Clearly the results of the 1D model are only a function of the soil properties and of the product $E_{p} I_{p}$ of the Young's modulus of the pile by the moment of inertia of the cross section but not explicitly of the pile radius. For a hollow pile the moment of inertia is not uniquely related to the radius and therefore in this case the actual size of the cavity has no effect on the results of the model if the moment of inertia is kept constant. This would also be the case for a Winkler foundation model with only horizontal springs.

Table 2-2. Variation of pile head displacement versus pile radius in different linear analysis methods with constant $E_{p} I_{p}$ for the pile

\begin{tabular}{ccccc}
\hline \multirow{2}{*}{$\begin{array}{c}\text { Pile radius } \\
(\mathrm{m})\end{array}$} & \multicolumn{4}{c}{ Pile head deflection $(\mathrm{mm})$} \\
\cline { 2 - 5 } & 3D pile FEM & 1D pile FEM & Consistent boundary matrix & Winkler foundation \\
\hline 0.50 & 32.5 & $68.3(110 \%)$ & $34.0(5 \%)$ & $25.2(23 \%)$ \\
1.00 & 27.6 & $68.3(148 \%)$ & $27.5(1 \%)$ & $25.0(10 \%)$ \\
2.00 & 20.9 & $68.3(227 \%)$ & $20.5(2 \%)$ & $24.3(17 \%)$ \\
\hline
\end{tabular}

Table 2-2 shows the results of the four models for hollow piles with the same $E_{p} I_{p}$ but radii of $0.5,1$ and $2 \mathrm{~m}$. The agreement between the $3 \mathrm{D}$ finite element model and the boundary matrix method is good in all three cases (about 3\% off in average.) As expected the results for the 1D pile model do not change. The results for the Winkler model vary slightly because of the rotational springs but the variation is still very small and the accuracy deteriorates as the radius of the pile decreases. To see when the results of the 1D model would become similar to those of the more accurate solutions the boundary matrix model was run for a larger number of radii going down to $0.01 \mathrm{~m}$. 
Figure 2-4 shows the variation of the head displacement with the pile radius in semi-log scale. The deflection predicted by the boundary matrix model for a pile with a radius of $0.01 \mathrm{~m}$ is $68.5 \mathrm{~mm}$ now in good agreement with the prediction of the $1 \mathrm{D}$ model. It is interesting to observe that the variation of the displacement for this hollow pile is approximately inversely proportional to the radius to the power 0.26 .

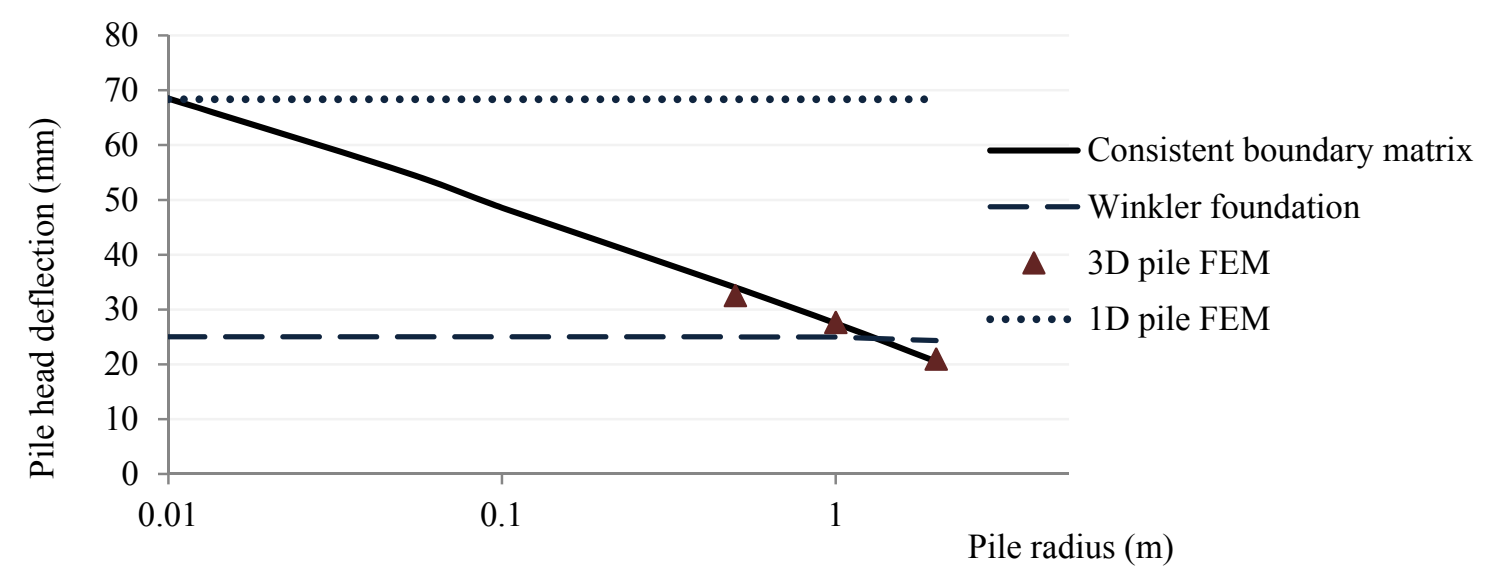

Figure 2-4. Variation of pile head displacement versus pile radius in linear analyses with constant $E_{p} I_{p}$ for the pile

\subsection{Nonlinear Analyses}

Three different models are used to conduct nonlinear analyses:

1) The 3D finite element model of the previous runs. In this case however the soil and the soil-pile interface are nonlinear. The finite element model, using ABAQUS, has the capability of taking into account the initial state of stresses in the soil mass. The initial conditions of stress are applied before the pile is installed and as a first step the effective body forces are calculated to account for geostatic equilibrium. The 
extreme static loads due to the performance of the turbine and wave and wind loading are applied then.

A $22 \mathrm{~m}$ long pile with a diameter of $4 \mathrm{~m}$ is modeled as a steel pipe using 4node quadrilateral shell elements with reduced integration. A $1 \mathrm{~m}$ long segment of the pile is considered to be above ground level to avoid the soil going over the pile. Linear elastic behavior is assumed for the pile.

For an actual soil profile it would be necessary to select the most appropriate nonlinear constitutive model and to determine the values of the required parameters defining the model from laboratory tests. For the purposes of this work and considering two hypothetical soils, a sand and a clay, a very simple Mohr Coulomb model, as implemented in the program ABAQUS, is used with the properties presented in Table 2-3. The finite element mesh of the $40 \mathrm{~m} \times 10 \mathrm{~m} \times 41 \mathrm{~m}$ soil mass is generated using isoparametric brick elements with reduced integration for the soil.

Table 2-3. Elastic-plastic properties of soil

\begin{tabular}{lccc}
\hline \multirow{2}{*}{ Parameter } & \multirow{2}{*}{ Symbol } & \multicolumn{2}{c}{ Value } \\
\cline { 3 - 4 } & & Sand & Clay \\
\hline Modulus of elasticity $(\mathrm{kPa})$ & $E_{\text {soil }}$ & $5.0 \mathrm{E} 4$ & $4.5 \mathrm{E} 4$ \\
Unit weight $\left(\mathrm{kN} / \mathrm{m}^{3}\right)$ & $\gamma_{\text {soil }}$ & 20.00 & 20.00 \\
Poisson ratio & $v_{\text {soil }}$ & 0.30 & 0.30 \\
Angle of internal friction $\left({ }^{\circ}\right)$ & $\phi_{\text {soil }}$ & 40.0 & - \\
Undrained shear strength $(\mathrm{kPa})$ & $S_{u}$ & - & 150.0 \\
\hline
\end{tabular}


The nonlinear behavior of the soil-pile contact is modeled using "contact pair" in ABAQUS. Tangential movement between the two parts, pile and surrounding soil, is allowed with a friction coefficient of 0.67 . In the radial direction, a "no separation" contact behavior is assumed. The pile outer surface is chosen as the "master surface" and the surface of the soil mass which is in contact with the pile is considered to be the "slave surface". The "small sliding" tracking approach is employed for the contact of the two bodies assuming that even if the two bodies undergo large motions, there is relatively little sliding of one surface along the other. An elastic-plastic Coulomb model is also used to describe the nonlinear behavior of the soil-pile contact. Figure 2-5 shows the deformation of soil with 3D nonlinear finite element model of pile foundation.

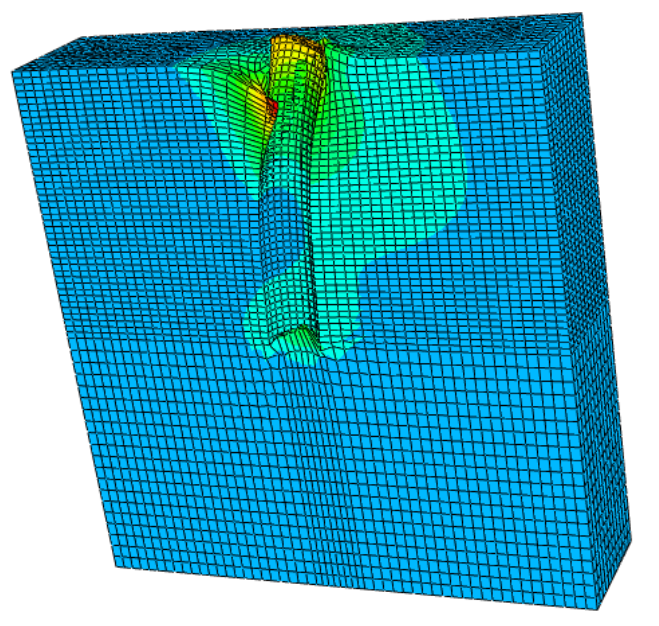

Figure 2-5. Deformed mesh of the pile foundation in 3D nonlinear analysis 
2) A model using the p-y curves is implemented specifically for this work. As indicated in the introduction section the $p-y$ curves were originally proposed by Matlock (1970) for soft clays under the water table and models for hard clays and sands were shortly after introduced by Reese et al. (1975.) In this work the sand and the hard clay model are used. The clay model requires the specification of a parameter $\varepsilon_{50}$ that has to be determined from experiments. Since the soil considered was not a real one on which experiments could be performed, a value of 0.005 , as recommended by Reese et al. (1975), is used. In the linear elastic range, for very small displacements, the initial stiffness of the springs representing the $p-y$ curves normally varies with depth. In this case however, to be consistent with the finite element model the initial stiffness value is considered to be constant with the depth and equal to $4 G$ as for the linear analyses with the Winkler foundation. Since the $p$ $y$ curves are in fact a form of the Winkler foundation model with only horizontal springs the solution in the elastic range would be only a function of the $E_{p} I_{p}$ and independent of the radius for a given moment of inertia. The nonlinear variation of the stiffness is on the other hand affected by the pile diameter. It should also be noticed that with the $p-y$ method there are nonlinear springs attached to the side of the pile but not at the bottom. One must decide therefore whether the pile tip is free, hinged or fixed. For long piles the difference between these three cases, when considering the pile head displacement, is negligible but in the present case the transfer length $l_{o}$ associated with the solution of the pile on a Winkler foundation is of the order of $10 \mathrm{~m}$ so the displacements for a hinged tip may be $25 \%$ smaller than 
for a free tip. For a linear analysis the assumption of a hinged tip may be more realistic but for the nonlinear one it is considered that the free end would be more appropriate. For the sake of comparison and to see the effects of such assumption the results are presented for both boundary conditions.

3) A model implementing the simple approach for lateral loads on piles (SALLOP) proposed by Briaud (1997.) It is a semi-theoretical or semi-empirical method in which the framework is theoretical but the factors in the theoretical equations are adjusted by comparison to some full-scale load tests. SALLOP uses two different theoretical solutions for infinitely long (flexible) piles and for short rigid piles in a Winkler uniform soil. Defining a transfer length $l_{o}=\left(4 E_{p} I_{p} / K_{s-s}\right)^{1 / 4}$ that is the typical parameter associated with the solution of a beam on elastic foundation, with $E_{p}=$ modulus of elasticity for the pile $(\mathrm{kPa}), I_{p}=$ moment of inertia for the pile $\left(\mathrm{m}^{4}\right)$, and $K_{s-s}=$ soil-spring constant $(\mathrm{kPa})$, the pile head displacement $y_{o}$ for long flexible piles $\left(H_{p} \geq 3 l_{o}\right)$ under a combined loading of a horizontal force and a moment at its head is (Briaud 1992)

$$
y_{o}=\frac{2 F_{o}}{l_{o} K_{s-s}}+\frac{2 M_{o}}{l_{o}^{2} K_{s-s}}
$$

where $F_{o}=$ horizontal force applied at the pile head $(\mathrm{kN}), M_{o}=$ moment applied at the pile head $(\mathrm{kNm}.) \quad K_{s-s}$ is defined as the ratio of the soil resistance at a specific depth to the horizontal pile displacement at the same depth. Briaud (1992) defined 
$K_{s-s}$ empirically by optimizing the comparison between the predicted deflection and the measured deflections, as

$$
K_{s-s}=2.3 E_{0}
$$

where $E_{o}=$ the preboring first load pressuremeter modulus within the zero-shear depth $l_{v}$. It is interesting to notice that a $K_{s-s}$ of $4 G_{\text {soil }}$ as used in the linear analyses with the Winkler model and for the initial branch of the $p-y$ curves corresponds to approximately $K_{s-s}=1.5 E_{\text {soil }}$. The depth $l_{v}$, referred to as the zero-shear depth, is obtained by setting the expression for the shear force in the pile equal to zero. For flexible piles $\left(H_{p} \geq 3 l_{o}\right)$

$$
l_{v}=l_{o} \tan ^{-1}\left(\frac{1}{1+\frac{2 M_{o}}{l_{o} F_{o}}}\right)
$$

For short rigid piles $\left(H_{p} \leq l_{o}\right)$ the zero-shear depth is expressed as

$$
D_{v}=\frac{F_{o} H_{p}^{2}}{3\left(F_{o} H_{p}+2 M_{o}\right)}
$$

The pile head displacement for short rigid piles $\left(H_{p} \leq l_{o}\right)$ is

$$
y_{o}=\frac{2\left(2 F_{o} H_{p}+3 M_{o}\right)}{K_{s-s} H_{p}^{2}}
$$

For the SALLOP calculations a linear interpolation between two values will be used if the pile length is between $l_{o}$ and $3 l_{o}$. Briaud (1992) also proposed the 
correlations between $N_{S P T}, S_{u}$ and $E_{o}$ when the standard penetration test (SPT) blow count $N_{S P T}$, or the undrained shear strength $S_{u}$ are available instead of $E_{o}$.

$$
\begin{aligned}
& E_{o}=383 N_{S P T} \\
& E_{o}=100 S_{u}
\end{aligned}
$$

For the pile with a radius of $2 \mathrm{~m}$, the 3D finite element model predicts a displacement of $40 \mathrm{~mm}$ in sand and $25.1 \mathrm{~mm}$ in clay. The corresponding results with the p-y curves are $38.2 \mathrm{~mm}$ and $37.5 \mathrm{~mm}$ with a free tip $(28.5 \mathrm{~mm}$ with a hinge at the bottom); with the SALLOP method 36.0 and $45.0 \mathrm{~mm}$. The three methods provide results in good agreement for the sand but there are larger differences for the clay particularly for the SALLOP approach and with a free tip for the $p-y$ curves.

The effect of the pile radius with a constant value of the $E_{p} I_{p}$ of the pile was again investigated for the nonlinear case. Table 2-4 and Figure 2-6 present the results of the three methods for radii of $0.5,1$ and $2 \mathrm{~m}$. Again since the SALLOP method is based purely on a Winkler foundation with horizontal springs the results are independent of the radius for a fixed $E_{p} I_{p}$. The $p-y$ curves give results that vary with the radius but less significantly than the 3D solution. It is interesting to notice that for the sand the best agreement is obtained for a radius of $2 \mathrm{~m}$. For the $0.50 \mathrm{~m}$ radius the prediction of the SALLOP method would be about $40 \%$ of the FEM result; with the $p$ - $y$ curve it would be about $62 \%$. For the clay on the other hand the best agreement between the three methods is obtained for the radius of $0.5 \mathrm{~m}$ (almost exactly the same results), whereas the discrepancy increases as the radius increases. The prediction with the SALLOP method 
is about $80 \%$ too large whereas that with the $p-y$ curves assuming a free tip is about $50 \%$ off for the $2 \mathrm{~m}$ radius.

Table 2-4. Variation of pile head displacement versus pile radius in different nonlinear analysis methods with constant $E_{p} I_{p}$ for the pile

\begin{tabular}{|c|c|c|c|c|c|c|c|}
\hline \multirow{4}{*}{$\begin{array}{l}\text { Pile } \\
\text { radius } \\
\text { (m) }\end{array}$} & \multicolumn{7}{|c|}{ Pile deflection at the ground level (mm) } \\
\hline & \multicolumn{3}{|c|}{ Sand } & \multicolumn{4}{|c|}{ Clay } \\
\hline & \multirow{2}{*}{$\begin{array}{c}\text { 3D } \\
\text { FEM }\end{array}$} & \multirow[b]{2}{*}{$p-y$} & \multirow{2}{*}{ SALLOP } & \multirow{2}{*}{$\begin{array}{c}3 \mathrm{D} \\
\text { FEM }\end{array}$} & \multicolumn{2}{|c|}{$p-y$} & \multirow{2}{*}{ SALLOP } \\
\hline & & & & & Free tip & Hinged tip & \\
\hline 0.50 & 91.0 & $57.0(35 \%)$ & $36.0(60 \%)$ & 45.5 & $45.3(1 \%)$ & $31.2(32 \%)$ & $45.0(1 \%)$ \\
\hline 1.00 & 60.6 & $43.5(29 \%)$ & $36.0(41 \%)$ & 33.9 & $40.5(20 \%)$ & $30.0(12 \%)$ & $45.0(33 \%)$ \\
\hline 2.00 & 40.0 & $38.2(5 \%)$ & $36.0(10 \%)$ & 25.1 & $37.5(50 \%)$ & $28.5(14 \%)$ & $45.0(80 \%)$ \\
\hline
\end{tabular}

It seems also that given the lack of a spring acting on the bottom face of the pile in the $p-y$ model, for the larger diameter pile the assumption of a hinged tip might be more realistic whereas for the smaller diameters it is better to consider a free tip. Considering the fact that the characteristics of the soils are not actually determined from laboratory tests but some of the parameters are chosen purely as logical values, and that a very simple nonlinear soil model was used, finding an exact agreement between the three methods would have been surprising. The fact that they provide results with the same order of magnitude for the range of pile diameters considered is encouraging. On the other hand it is important to notice the effect of the pile radius on the foundation stiffness beyond the value of the $E_{p} I_{p}$, something that would occur irrespective of the constitutive model used. Obtaining a very good agreement for a given pile radius with a more refined selection of the nonlinear soil model and of the soil parameters will not guarantee similar accuracy for other values of the radius and the same soil. 


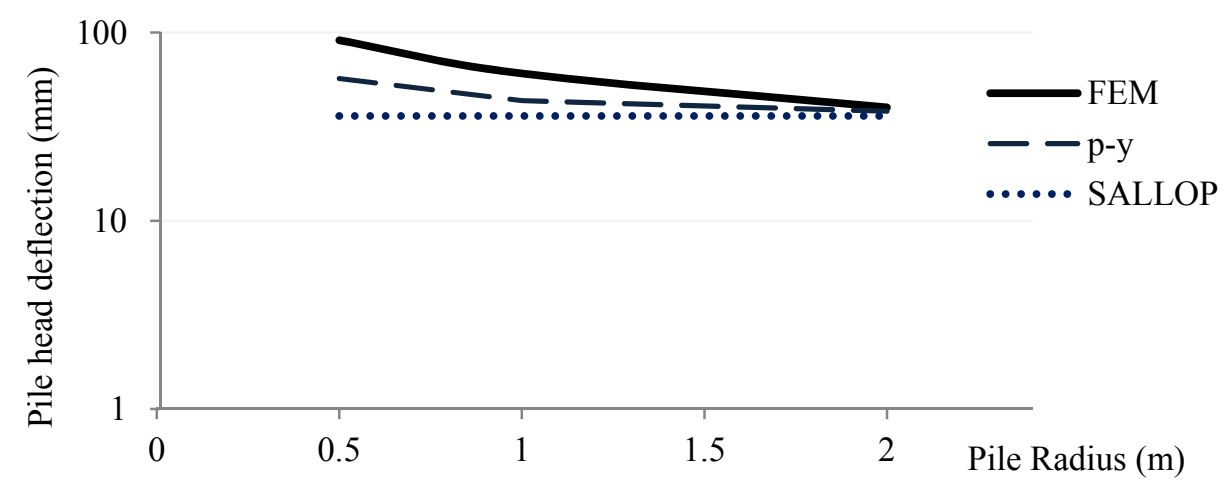

(a)

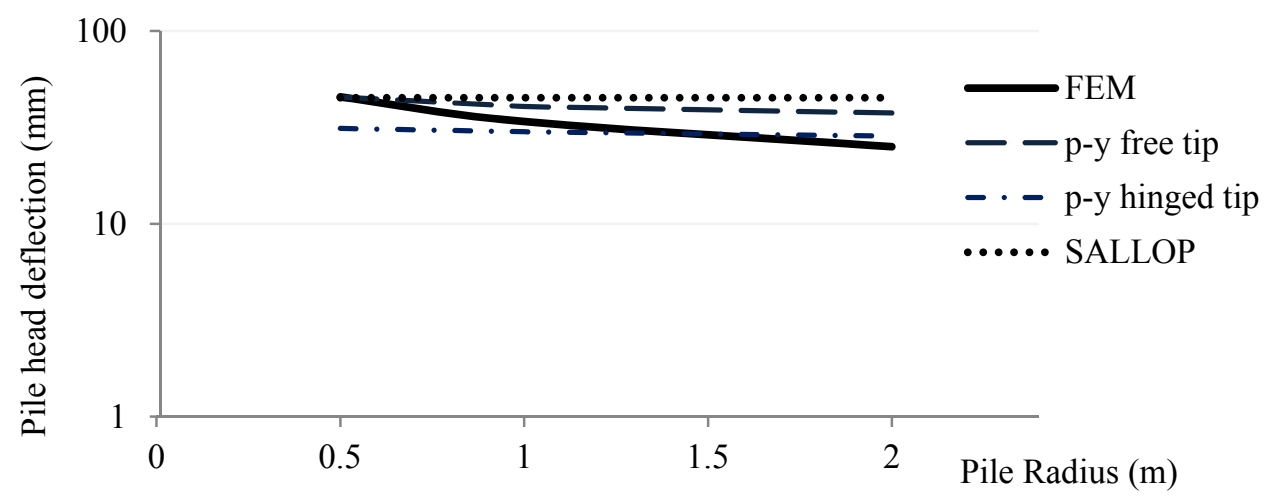

(b)

Figure 2-6. Variation of pile head displacement versus pile radius in nonlinear analyses for (a) sand and (b) clay with constant $E_{p} I_{p}$ for the pile

\subsection{Conclusions}

The effect of the pile radius on its lateral behavior in the linear elastic range was studied using various analysis procedures assuming a constant pile stiffness $\left(E_{p} I_{p}\right)$ and different pile radii for hollow piles: a three dimensional (3D) ABAQUS finite element (FE) model, a model with the soil reproduced with $3 \mathrm{D}$ elements but the pile represented by a line, a model using a consistent boundary matrix and a Winkler foundation model. The results show that the pile head lateral deflection is not only a function of $E_{p} I_{p}$ but also 
of the pile radius. It decreases considerably as the pile radius increases while $E_{p} I_{p}$ is maintained constant. Modeling a pile as a one dimensional (1D) line with beam-column elements, as done sometimes in the literature, results in a smaller contribution of the surrounding soil to the lateral stiffness of the pile and an increase of up to $200 \%$ in the maximum displacement of the pile head.

Nonlinear analyses were next conducted using the 3D FE models of the soil and pile employing ABAQUS for a sand and a clay. The static (monotonic) calculations were conducted for an extreme lateral load and bending moment. A Mohr-Coulomb constitutive model was used for the generic soils. The nonlinear contact between the pile and the soil were accounted for using some of the tools available in ABAQUS. The results were compared to those provided by the use of $p-y$ curves for sand and hard clay and with the SALLOP method suggested by Briaud (1997.) Both the $p-y$ model for sand and the SALLOP method provide reasonable answers for the pile with a radius of $2 \mathrm{~m}$ but the accuracy deteriorates for smaller radii, particularly for the SALLOP method where the results are independent of the radius for a fixed value of $E_{p} I_{p}$. For the clay the $p-y$ curves assuming a free tip and the SALLOP predictions are good for the smaller diameter pile (radius of $0.5 \mathrm{~m}$ ) but deteriorate for larger diameters. It appears that for these cases with the $p-y$ method the assumption of a pile hinged at the bottom would provide better results.

The study conducted uses the 3D nonlinear FE analysis as an accurate analysis for the pile sizes of interest in relation to the foundations of offshore wind turbines to assess other, simpler models. It indicates that when using common simple models and 
particularly if the pile is modeled as a line, neglecting the size of the soil cavity, the results may be inaccurate. 


\section{PROBABILISTIC DEMAND MODELS AND FRAGILITY ESTIMATES FOR OFFSHORE WIND TURBINES SUBJECT TO DAY-TO-DAY LOADS}

\subsection{Introduction}

Reliable power production of a wind turbine is one of the key factors to reduce the cost of energy. Walford (2006) shows how improving system reliability is critical to reduce the operation and maintenance cost of wind turbines. Providing adequate reliability can help reduce the need for costly repairs and downtime. Furthermore, an accurate assessment of the reliability of wind turbines can be used for a reliability-based optimal design that minimizes construction and maintenance costs while maintaining minimum reliability requirements.

Several aeroelastic simulation codes are used in the wind energy industry to simulate fatigue, aerodynamics and structural dynamic response. For example, FAST and GH Bladed are two commonly used simulators that are found accurate in simulating wind turbine aerodynamics and estimating the fatigue and extreme loadings. However, they are not capable of continuous modeling the foundation, incorporating the dynamic soil-structure interaction.

Mono-piles are common foundations for offshore wind turbine support structures installed in water depths less than 30 meters, which is the focus of this dissertation. Analysis of laterally loaded single piles is in general based on Winkler (elastic) foundation models, or continuous models accounting for the coupling of forces and displacements in the soil along the pile. Winkler foundation models are popular because 
of their simplicity and reasonable accuracy. For nonlinear analyses the $p-y$ method developed first by Matlock (1970) is the most commonly used one. Reese and Wang (2008) used a design method for pile foundations of wind turbine support structures based on $p-y$ curves to take soil-structure interaction into account, employing equivalent springs. However, investigation of the behavior of laterally loaded piles using a 3D nonlinear FE model in the previous section showed that, depending on the pile diameter and soil type, using common simple models, such as $p-y$ method and particularly modeling the pile using one dimensional beam-column elements may result in inaccurate responses. This is true in particular for the pile sizes typical of foundations of offshore wind turbines. On the other hand, a complete nonlinear finite element analysis of the support structure and the foundation can be quite expensive and time consuming.

To address the limitations of prevailing approaches, this section develops simplified probabilistic models to predict the deformation, shear and moment demands on the support structure of offshore wind turbines subject to day-to-day wind, wave, current and turbine operational loadings. The probabilistic models properly account for the nonlinear soil-structure interaction as well as the inherent uncertainties, including the statistical uncertainty (associated with the finite sample size) and the modeling errors (associate with the selection of the variables in the models and the model forms.) Due to the lack of available field experiment data on offshore wind turbines, a virtual experiment database is generated from the results of $3 \mathrm{D}$ nonlinear finite element analyses and used to calibrate the probabilistic models. 
The next subsection explains the generation of the virtual experiment data, which are later used to calibrate the probabilistic models. Third subsection discusses the general formulation of probabilistic demand models and then following this formulation, deformation, shear and moment demand models are developed. Finally, the fragility estimates are presented for an example offshore wind turbine support structure for given values of two demand parameters, namely, the mean wind speed, $W_{s}$, and significant wave height, $H_{s}$. Where $W_{s}$ is defined as the wind speed average over a time window of 10 minutes, and $H_{s}$ is defined as the mean wave height (through to crest) of the highest third of the waves.

\subsection{Virtual Experiment Data}

A set of representative configurations is used to generate a virtual experiment database that is later used to calibrate the proposed probabilistic demand models. Nonlinear analyses are conducted for each configuration using detailed 3D finite element models of the support structure and the foundation with consideration of the nonlinear soil-pile interaction. representative configurations are selected using an experimental design to maximize the information content of the considered configurations and minimize the computation costs associated with running the detailed nonlinear finite element analyses.

\subsubsection{Experimental Design}

In the "classical" design of physical experiments, a random variation is accounted for by spreading the sample points out in the design space and by taking multiple (replicated) 
data points (Simpson et al., 2001.) However, when it comes to deterministic computer experiments, there is no random error and no replication is required. According to Simpson et al. (2001), the design space is defined as the region bounded by the upper and lower limits of each design (input) variable being studied and the sample points should be chosen to fill the design space for computer experiments such that they spread as far from each other as possible. There are several "space filling" design methods in the literature; here the Latin hypercube sampling technique introduced by McKay et al. (1979) is used to select representative configurations of the support structure. Latin hypercube sampling technique maximizes the minimum distance between sample points, while the range of each variable, $x_{i}$, is divided into $\mathrm{N}$ strata of equal marginal probability $1 / \mathrm{N}$, therefore, this method ensures that the sampling has a good coverage of the design space. A total of 100 configurations are generated. Variables considered to characterize each wind turbine configuration and their ranges are presented in Table 3-1.

\subsubsection{Analytical Modeling}

Detailed 3D nonlinear FE models developed in ABAQUS are used to simulate the nonlinear response of typical offshore wind turbine support structures subject to day-today wind, wave, current and turbine operational loading. The finite element model of the support structure properly accounts for the influence of soil-structure interaction as well as nonlinearity of the soil behavior. 
Table 3-1. Geometrical and mechanical properties used in experimental design

\begin{tabular}{|c|c|c|c|c|c|}
\hline \multicolumn{2}{|l|}{ Property } & Symbol & \multicolumn{2}{|l|}{ Ranges } & Unit \\
\hline \multicolumn{2}{|l|}{ Rotor diameter } & $R D$ & \multicolumn{2}{|l|}{$40-126$} & $\mathrm{~m}$ \\
\hline \multicolumn{2}{|l|}{ Tower height } & $H_{H}$ & \multicolumn{2}{|l|}{$40-90$} & $\mathrm{~m}$ \\
\hline \multicolumn{2}{|l|}{ Tower top diameter } & $d_{t}$ & \multicolumn{2}{|l|}{$1.9-4.0$} & $\mathrm{~m}$ \\
\hline \multicolumn{2}{|c|}{ Tower diameter to wall thickness ratio } & $\alpha_{t}$ & \multicolumn{2}{|l|}{$100-200$} & -- \\
\hline \multicolumn{2}{|l|}{ Water depth } & $H_{W r}$ & \multicolumn{2}{|l|}{$20-30$} & $\mathrm{~m}$ \\
\hline \multicolumn{2}{|l|}{ Steel type } & $S T$ & \multicolumn{2}{|c|}{$\mathrm{S} 235, \mathrm{~S} 275, \mathrm{~S} 355$} & -- \\
\hline \multicolumn{2}{|l|}{ Material damping ratio } & $\xi$ & \multicolumn{2}{|l|}{0.05} & -- \\
\hline \multirow{2}{*}{ Support structure vibration period } & First mode & $T_{n}$ & \multicolumn{2}{|l|}{$0.9-11.9$} & $\mathrm{~s}$ \\
\hline & Second mode & $T_{s}$ & \multicolumn{2}{|l|}{$0.5-3.6$} & $\mathrm{~S}$ \\
\hline \multicolumn{2}{|l|}{ Pile diameter } & $d_{p}$ & \multicolumn{2}{|l|}{$3.0-6.0$} & $\mathrm{~m}$ \\
\hline \multicolumn{2}{|l|}{ Pile penetration } & $H_{p}$ & \multicolumn{2}{|l|}{$10-50$} & $\mathrm{~m}$ \\
\hline \multicolumn{2}{|l|}{ Pile diameter to wall thickness ratio } & $\alpha_{p}$ & \multicolumn{2}{|l|}{$50-100$} & -- \\
\hline \multicolumn{2}{|l|}{ Soil modulus of elasticity } & $E_{\text {soil }}$ & \multicolumn{2}{|l|}{$13-200$} & $\mathrm{MPa}$ \\
\hline \multicolumn{2}{|l|}{ Friction between pile and soil } & $f r_{p-s}$ & \multicolumn{2}{|l|}{$0.2-0.3$} & -- \\
\hline \multicolumn{2}{|l|}{ Soil type } & -- & Clay & Sand & \\
\hline \multicolumn{2}{|l|}{ Soil cohesion } & $C_{\text {soil }}$ & $10-200$ & $0-80$ & $\mathrm{kPa}$ \\
\hline \multicolumn{2}{|l|}{ Soil friction angle } & $\phi_{\text {soil }}$ & $10-25$ & $35-45$ & $\circ$ \\
\hline \multicolumn{6}{|l|}{ Loading Parameters } \\
\hline \multicolumn{2}{|l|}{ Mean wind speed } & $W_{s}$ & $3.0-30$ & & $\mathrm{~m} / \mathrm{s}$ \\
\hline Turbulence intensity & & $I T_{w}$ & $0-0.16$ & & -- \\
\hline Significant wave height & & $H_{s}$ & $1.0-10$ & & $\mathrm{~m}$ \\
\hline Wave peak period & & $T_{p}$ & $3.6 \sqrt{H_{s}}$ & $5.0 \sqrt{H_{s}}$ & $\mathrm{~S}$ \\
\hline Rated wind speed & & $W_{\text {s-rated }}$ & $10.3-11$. & & $\mathrm{m} / \mathrm{s}$ \\
\hline
\end{tabular}

Considering the symmetry of a wind turbine support structure and foundation, half of the support structure is modeled to reduce the analysis time. Figure 3-1 shows the detailed 3D FE model of a sample wind turbine support structure created in ABAQUS. The tubular steel tower and pile foundation are modeled using 3D shell elements. 3D solid elements are used to model the soil mass. Nonlinearity of the foundation is considered explicitly by defining the nonlinear soil behavior with a Mohr-Coulomb plasticity model and soil-pile interaction. 
ABAQUS supports "contact pair" that is a formulation used to model the nonlinearity of the contact between the pile and surrounding soil. A tangential movement is allowed with a friction coefficient ranging from 0.2 to 0.3 . A "no separation" contact behavior is assumed in the radial direction. The outer surface of the pile is chosen as the "master surface" and the surface of the soil mass that is in contact with the pile is considered to be the "slave surface". A "small sliding" tracking approach is used for the contact between the two bodies assuming that there is always relatively little sliding of one surface along the other, even if the two parts undergo large displacements. The nonlinearity of the soil-pile contact behavior is modeled using an elastic-plastic Coulomb model.

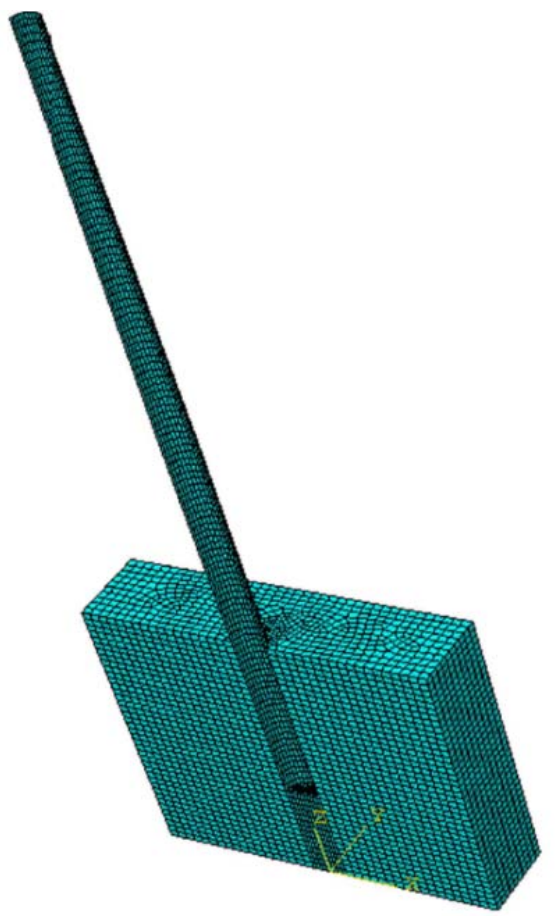

Figure 3-1. Finite element model of a sample wind turbine support structure in ABAQUS 
The wind turbine simulator FAST is used to simulate the aerodynamics of the turbine. FAST (Fatigue, Aerodynamics, Structures, and Turbulence) is developed at the National Renewable Energy Laboratory (NREL) by Jonkman and Buhl Jr. (2005.) It is a comprehensive simulator capable of predicting both the extreme and fatigue loads of two- and three-bladed horizontal-axis wind turbines (Jonkman and Buhl Jr. 2005.) TurbSim, a turbulence simulator developed by Jonkman (2009), is used to generate the time history of the wind speed later used as an input for FAST. TurbSim is a stochastic, full-field, turbulent-wind simulator. It uses a statistical or empirical model (as opposed to a physics-based model) to numerically simulate time series of three-component windspeed vectors (Jonkman 2009.) TurbSim supports the Kaimal spectrum proposed by Kaimal et al. (1972) to simulate wind turbulence. The spectrum in the normalized form is given as

$$
\frac{f_{w} S_{w i}\left(f_{w}\right)}{\sigma_{w, i}^{2}}=\frac{4 f_{w} L_{w i} / W_{h u b}}{\left(1+6 f_{w} L_{w i} / W_{h u b}\right)^{5 / 3}}
$$

where $f_{w}=$ frequency in Hertz, $S_{w i}=$ single-sided velocity component spectrum, $\sigma_{w, i}=$ standard deviation of the $i^{\text {th }}$ velocity component, and $L_{w i}=$ velocity component integral scale parameter, and $W_{\text {hub }}=$ the wind speed at the hub height. The following exponential coherence model suggested by the International Electrotechnical Commission standard (IEC 2005) is used in conjunction with the Kaimal spectrum to account for the spatial correlation structure of the longitudinal velocity component:

$$
\operatorname{Coh}\left(\alpha_{w}, f_{w}\right)=\exp \left\{-12\left[\left(f_{w} \alpha_{w} / W_{h u b}\right)^{2}+\left(0.12 \alpha_{w} / L_{s c}\right)^{2}\right]^{0.5}\right\}
$$


where $\alpha_{w}=$ the magnitude of the projection of the separation vector between the two points on to a plane normal to the average wind direction, and $L_{s c}=$ the coherence scale parameter.

As an internal subroutine, FAST also uses another computer program AeroDyn (Laino and Hansen 2002) to compute the aerodynamic forces on the rotating blades. The results of the FAST simulation are then applied to the tower as an external loading in addition to the wave and current loads that are modeled separately as described next.

A linear irregular wave model, given as the superposition of linear regular waves propagating at different frequencies, is used to simulate the stochastic ocean waves. This model is based on the solution of the Laplace equation in terms of the velocity potential, given by the following equation (Dean and Dalrymple 1991):

$$
\Omega=\sum_{m=1}^{M}-A_{m} \frac{g}{\omega_{m}} \frac{\cosh k_{m}\left(H_{w r}+z_{w r}\right)}{\cosh k_{m} H_{w r}} \sin \left(\omega_{m} t-k_{m} X_{w r}-\phi_{m}\right)
$$

where $\Omega=$ the velocity potential at a point with coordinates $x_{w r}$ and $z_{w r}$ (see Figure 32.) Note that the origin of the $z_{w r}$ axis is selected at the mean sea level (MSL); also the centerline of the turbine mono-pile is assumed to be located at $x_{w r}=0 . \omega_{m}=$ the frequency of the $m^{\text {th }}$ wave component, determined by solving the dispersion equation of $\omega_{m}^{2}=g k_{m} \tanh \left(k_{m} H_{w r}\right), g=$ the acceleration of gravity, $H_{w r}=$ water depth, and $k_{m}=2 \pi / L_{m}=$ the wave number, where $L_{m}=$ the wave length. $A_{m}=$ the amplitude of the $m^{\text {th }}$ wave component, $\phi_{m}=$ the associated random phase assumed uniformly distributed over $[0,2 \pi]$ and finally $t$ represents the time of simulation. 


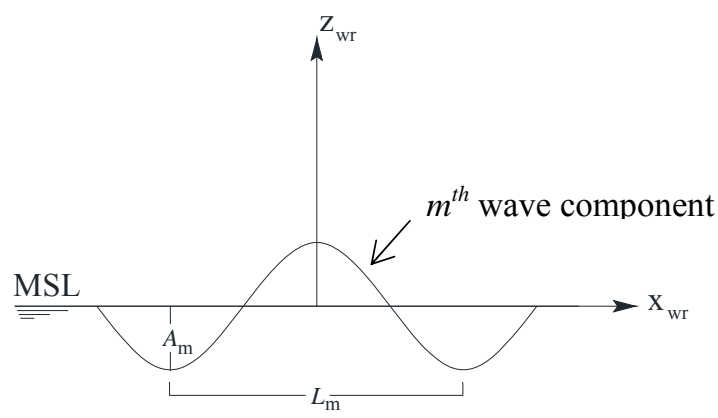

Figure 3-2. Schematic illustration of wave relative to the mean sea level (MSL)

Following Det Norske Veritas guideline for design of wind turbines (DNV/Risø 2002), the Morison's equation is used to determine the hydrodynamic forces. The horizontal force on a vertical element $d z$ of the structure at level $z$ is expressed as (Dean and Dalrymple 1991)

$$
d F=d F_{D}+d F_{I}=\frac{1}{2} C_{D} \rho_{w r} d_{P} V_{w r}\left|V_{w r}\right|+C_{I} \rho_{w r} \frac{\pi d_{P}^{2}}{4} \dot{V}_{w r}
$$

where $d F_{D}$ and $d F_{I}=$ the drag and inertia forces, respectively, $C_{D}=$ drag coefficient, $C_{I}=$ inertia coefficient, $d_{P}=$ pile diameter, and $\rho_{\mathrm{wr}}=$ density of water. The horizontal water particle velocity, $V_{w r}$, and acceleration, $\dot{V}_{w r}$, are determined as $V_{w r}=-\partial \Omega / \partial x_{w r}$ and $\dot{V}_{w r}=\partial V_{w r} / \partial t$, respectively. Eq. (3-4) neglects the velocity and acceleration of the structure. The current load is predicted using Morison's equation (Eq. 3-4), where, $V_{w r}$, is taken as the resultant of the combined current and wave velocity. Figure 3-3 shows a schematic representation of how ABAQUS, FAST and TurbSim are combined to model the dynamic behavior of a wind turbine system. 


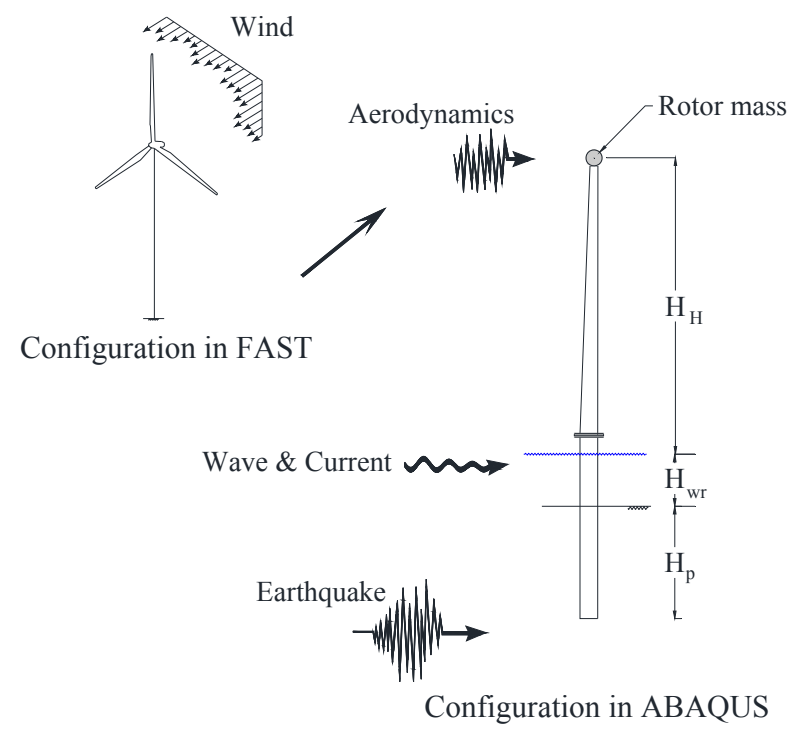

Figure 3-3. Typical offshore wind turbine support structure configuration

\subsubsection{Equality and Lower Bound Data}

Finite element analyses for large deformations are sensitive to how the solution method handles large displacements and second order effects. As a result, the outcomes are not always accurate. For this reason, a drift threshold of $5 \%$ is set for analyses to be considered precise. Then following Gardoni et al. (2002) and Ramamoorthy et al. (2006), the data from the virtual experiments are divided into equality and lower bound data. An equality datum is such that the value of the recorded quantity of interest (deformation, shear force, or bending moment) in the 3D FE analysis is believed to be accurate. A lower bound datum is such that an accurate record of the quantity of interest is not available and only a lower bound of the true value is available.

In this section, the deformation, shear and moment data are considered as equality data, if the maximum drift during a time history analysis does not exceed $5 \%$. If 
an analysis produces a drift that exceeds $5 \%$, then the maximum value of deformation, shear and moment that occurs prior to reaching the $5 \%$ drift are considered as lower bound data for the deformation, shear and moment, respectively. With this approach, the data from analyses that lead to large deformations are included without letting inaccurate values wrongfully influence the model parameters (Bisadi et al. 2006.)

\subsection{Probabilistic Demand Models}

A probabilistic demand model relates the demand on the structural component to the properties of the considered system and the intensity measures of demand(s) and hazard(s) while accounting for the uncertainties inherent in the demand model. Ideally a model should incorporate all the available sources of information including the rules of physics and mechanics, and experimental and field data when they are available. Following Gardoni et al. (2002 and 2003), the probabilistic demand models are formulated by adding a correction term to selected deterministic demand models. A probabilistic demand model is formulated as

$$
D_{k}\left(\mathbf{x}, \mathbf{w}, \boldsymbol{\Theta}_{k}\right)=\hat{d}_{k}(\mathbf{x}, \mathbf{w})+\gamma_{k}\left(\mathbf{x}, \mathbf{w}, \boldsymbol{\theta}_{k}\right)+\sigma_{k} \varepsilon_{k}
$$

where $D_{k}\left(\mathbf{x}, \mathbf{w}, \boldsymbol{\Theta}_{k}\right)=k^{\text {th }}$ probabilistic demand model, in which $k=\delta, v$ or $m$ stands for deformation, shear or moment, respectively, $\mathbf{x}=$ material properties, structural dimensions and boundary conditions, $\mathbf{w}=$ a vector of measures of the external loading, including wind and wave properties, $\boldsymbol{\Theta}_{k}=\left(\boldsymbol{\theta}_{k}, \sigma_{k}\right)$, in which $\boldsymbol{\theta}_{k}=\left[\theta_{k i}\right]=$ vector of unknown model parameters and $\sigma_{k}=$ standard deviation of the model error $\sigma_{k} \varepsilon_{k}$, 
$\hat{d}_{k}(\mathbf{x}, \mathbf{w})=$ selected deterministic demand model, $\gamma_{k}\left(\mathbf{x}, \mathbf{w}, \boldsymbol{\theta}_{k}\right)=$ correction term for the bias inherent in the deterministic model, $\varepsilon_{k}=$ random variable with zero mean and unit variance. In formulating the model, a logarithmic transformation of the data is employed to satisfy the homoskedasticity assumption ( $\sigma_{k}$ is constant), the normality assumption ( $\varepsilon_{k}$ has the normal distribution), and the additive form used in Eq. (3-5.)

The correction term $\gamma_{k}\left(\mathbf{x}, \mathbf{w}, \boldsymbol{\theta}_{k}\right)$, added to correct for the potential bias in deterministic model by incorporating the missing terms in $\hat{d}_{k}(\mathbf{x}, \mathbf{w})$, is written as

$$
\gamma_{k}\left(\mathbf{x}, \mathbf{w}, \boldsymbol{\theta}_{k}\right)=\sum_{i=1}^{p} \theta_{k i} h_{k i}(\mathbf{x}, \mathbf{w})
$$

where $h_{k i}(\mathbf{x}, \mathbf{w})=$ normalized explanatory functions that might be significant in correcting $\hat{d}_{k}(\mathbf{x}, \mathbf{w})$, and $p=$ the number of unknown model parameters. The model parameters, $\boldsymbol{\Theta}_{k}=\left(\boldsymbol{\theta}_{k}, \sigma_{k}\right)$, are estimated by a Bayesian updating method and using the results from the detailed finite-element analyses.

An offshore wind turbine support structure can fail in shear, moment or excessive deformations. This section develops probabilistic models for the deformation, shear and moment demands of offshore wind turbines with horizontal axis. More specifically, the wind turbines of interest in this study are rated between 0.5 and 5 megawatts, supported by a tubular steel tower and a steel mono-pile foundation, installed in water depths less than 30 meters and subject to day-to-day turbine operational loads, as well as wind, wave and current loading. 


\subsubsection{Deterministic Demand Model}

An ideal deterministic demand model $\hat{d}_{k}(\mathbf{x}, \mathbf{w})$ should be simple and yet accurate, and ideally commonly used in practice to account for the current knowledge and facilitate the acceptance of the proposed probabilistic model. For this reasons, the deterministic prediction from FAST is used. For this purpose, the structural model in FAST consists of nine rigid bodies (the earth, support platform, base plate, nacelle, armature, gear system, hub, tail and structure furling with the rotor) and five flexible bodies (the tower, drive shaft and three blades) that are related through 24 degrees of freedom.

TurbSim simulates the wind turbulence as explained in Section 3.2.2 and generates the time history of the wind speed at the hub height that is applied to the simulated turbine in FAST. As opposed to the FAST simulations that are used for generating the virtual experimental data, for the purpose of modeling the deterministic demand, wave and current loads are calculated internally in FAST. To model linear irregular waves, FAST supports JONSWAP/Pierson-Moskowitz spectrum and then uses the Morison's equation to determine the hydrodynamic forces on the tower.

\subsubsection{Model Correction}

To correct for the bias inherent in the deterministic model, the additive correction term, $\gamma_{k}\left(\mathbf{x}, \mathbf{w}, \boldsymbol{\theta}_{k}\right)$, is developed as presented in Eq. (3-6.) Ideal selection of candidate explanatory functions $h_{k i}(\mathbf{x}, \mathbf{w})$ is based on the laws of mechanics to improve the model by incorporating missing terms in deterministic model. The term $h_{k 1}(\mathbf{x}, \mathbf{w})=1$ is selected 
to capture a potential constant bias in the model that is independent of the variables $\mathbf{x}$ and $\mathbf{w}, h_{k 2}(\mathbf{x}, \mathbf{w})=\hat{d}_{k}(\mathbf{x}, \mathbf{w})$ to capture any possible under- or over-estimation of the deterministic model. Table 3-2 summarizes the candidate explanatory functions selected for demand models. To characterize the influence of wind and wave parameters, $h_{k 3}-h_{k 6}$ are selected as normalized functions of $W_{s}, I T_{w}, H_{s}$, and $T_{p}$, respectively. The explanatory function $h_{k 7}$ is considered to capture the possible influence of the rotor diameter. In addition, $h_{k 8}-h_{k 11}$ are considered to capture the possible influence of the foundation stiffness, $k_{f}$, which is not included in the deterministic model.

The foundation stiffness $k_{f}$ is computed using $p-y$ curves. The $p-y$ method uses an elastic beam-column member to model the pile and nonlinear horizontal springs to represent the soil reactions. The $p-y$ curves describe the nonlinear behavior of the soil springs. They were originally proposed by Matlock (1970) for soft clays under the water table and Reese et al. (1975) shortly after introduced models for hard clays and sands. Sand, soft clay and hard clay models are used in this study. The clay model requires the specification of a parameter $\varepsilon_{50}$ that is assumed to be 0.005 , as recommended by Reese et al. (1975.)

To develop parsimonious probabilistic demand models, the desire is to keep only the explanatory functions that are strictly needed. Therefore, a model selection process is used to identify the important explanatory functions among the candidates presented in Table 3-2. 
Table 3-2. Explanatory functions for demand models

\begin{tabular}{lll}
\hline Explanatory function & Formula & \multicolumn{1}{c}{ Parameters } \\
\hline$h_{k 1}$ & 1 & $k=\delta, v$ or $m$ \\
$h_{k 2}$ & $\hat{d}_{k}$ & $\hat{d}_{k}=$ Deterministic deformation, shear or moment demand \\
$h_{k 3}$ & $\ln \left(W_{s} T_{n} / H_{H}\right)$ & $H_{H}=$ Hub height \\
$h_{k 4}$ & $\ln \left(I T_{w}\right)$ & \\
$h_{k 5}$ & $\ln \left(H_{s} / H_{H}\right)$ & \\
$h_{k 6}$ & $\ln \left(T_{p} / T_{n}\right)$ & \\
$h_{k 7}$ & $\ln \left(R D / H_{H}\right)$ & \\
$h_{k 8}$ & $\ln \left(C_{s} / C_{s \max }\right)$ & $C_{s}=$ Soil shear wave velocity; $C_{s \max }=194.594 \mathrm{~m} / \mathrm{s}$ \\
$h_{k 9}$ & $\ln \left(C_{\text {soil }} / E_{\text {soil }}\right)$ & \\
$h_{k 10}$ & $\ln \left[\tan \left(\phi_{\text {soil }}\right)\right]$ & \\
$h_{k 11}$ & $\ln \left(k_{t} / k_{f}\right)$ & $k_{t}=$ Tower stiffness \\
\hline
\end{tabular}

\subsubsection{Model Selection}

A stepwise deletion procedure is used for reducing the number of terms in $\gamma_{k}\left(\mathbf{x}, \mathbf{w}, \boldsymbol{\theta}_{k}\right)$ to achieve a compromise between the simplicity (few correction terms) and accuracy (small $\sigma)$ of the model. The stepwise deletion procedure used in this section follows the procedure developed by Gardoni et al. (2002.) For completeness, a brief summary of the procedure is provided here. The procedure starts with a comprehensive candidate form of $\gamma_{k}\left(\mathbf{x}, \mathbf{w}, \boldsymbol{\theta}_{k}\right)$ and then is simplified by deleting unimportant terms one at the time. At each step, first the posterior statistics of the model parameters $\boldsymbol{\Theta}_{k}$ are assessed using a Bayesian approach. Then the term $h_{k j}$ whose coefficient $\theta_{k j}$ has the largest posterior coefficient of variation (COV) is identified. The term $h_{k j}$ is the least informative among all the explanatory functions in the $k^{\text {th }}$ demand model and might be dropped from 
$\gamma_{k}\left(\mathbf{x}, \mathbf{w}, \boldsymbol{\theta}_{k}\right)$. In the next step, the reduced model is re-assessed by estimating its remaining parameters and the value of $\sigma_{k}$, which captures the model accuracy, is checked. If the value of $\sigma_{k}$ grows of an unacceptable amount from the value in the previous step, then the term $h_{k j}$ should not have been deleted and the most parsimonious model is the one at the previous step. It is noted that the stepwise deletion procedure used in this section was developed specifically to identify the most parsimonious model when the data include lower or upper bound data and traditional deletion procession cannot be used.

\subsubsection{Bayesian Updating}

A Bayesian approach is used to estimate the unknown model parameters $\boldsymbol{\Theta}_{k}$ using the data from the virtual experiments. The updating rule can be written as (Box and Tiao 1992)

$$
f\left(\boldsymbol{\Theta}_{k}\right)=\kappa L\left(\boldsymbol{\Theta}_{k}\right) p\left(\boldsymbol{\Theta}_{k}\right)
$$

where $p\left(\boldsymbol{\Theta}_{k}\right)=$ the prior distribution of $\boldsymbol{\Theta}_{k}$ that reflects the state of knowledge about $\boldsymbol{\Theta}_{k}$ , $L\left(\boldsymbol{\Theta}_{k}\right)=$ the likelihood function that represents the objective information on $\boldsymbol{\Theta}_{k}$ contained in the virtual experiment database, $\kappa=$ a normalizing factor, and $f\left(\boldsymbol{\Theta}_{k}\right)=$ the posterior distribution of $\boldsymbol{\Theta}_{k}$ that represents the updated state of knowledge about $\boldsymbol{\Theta}_{k}$. The posterior distribution $f\left(\boldsymbol{\Theta}_{k}\right)$ incorporates both the previous information about $\boldsymbol{\Theta}_{k}$ included in $p\left(\boldsymbol{\Theta}_{k}\right)$ and the new data included in $L\left(\boldsymbol{\Theta}_{k}\right)$. In this section, due to the lack of prior information on the unknown parameters, a non-informative prior in the form of 
$p\left(\boldsymbol{\theta}_{\delta}, \sigma_{\delta}\right) \propto \sigma_{\delta}^{-1}$ (Box and Tiao 1992) is used in the Bayesian approach. However, any knowledge based on prior experience could be used to refine the model. Furthermore, application of the updating rule in Eq. (12) can be repeated to update our present state of knowledge as new information on $\boldsymbol{\Theta}_{k}$ becomes available.

The likelihood function is proportional to the conditional probability of making the observations for a given value of $\boldsymbol{\Theta}_{k}$ and following Gardoni et al. (2002) it is written as

$$
L\left(\boldsymbol{\Theta}_{k}\right) \propto \prod_{\text {equality data }}\left\{\frac{1}{\sigma_{k}} \varphi\left[\frac{r_{k i}\left(\boldsymbol{\theta}_{k}\right)}{\sigma_{k}}\right]\right\} \times \prod_{\text {lower bound data }} \Phi\left[-\frac{r_{k i}\left(\boldsymbol{\theta}_{k}\right)}{\sigma_{k}}\right]
$$

where $r_{k i}\left(\boldsymbol{\theta}_{k}\right)=D_{k i}-\hat{d}_{k}\left(\mathbf{x}_{i}, \mathbf{w}_{i}\right)-\gamma_{k}\left(\mathbf{x}_{i}, \mathbf{w}_{i}, \boldsymbol{\theta}_{k}\right), D_{k i}=$ the $i^{\text {th }}$ observation of the $k^{\text {th }}$ demand for a given $\mathbf{x}_{i}$ and $\mathbf{w}_{i}$.

\subsubsection{Probabilistic Demand Models}

\subsubsection{Deformation Demand Model}

The deformation demand model is formulated in terms of the natural logarithm of the drift demand defined as deformation demand at the top of the tower, $\Delta$, normalized by the hub height $H_{H}$. The logarithmic transformation is used to satisfy the homoskedasticity, normality and additivity assumptions. The stepwise deletion procedure described in the Section 3.3.3 is then used to select the most parsimonious model. Figure 3-4 summarizes the stepwise deletion process for the deformation demand model. At each step, solid dots show the posterior COVs of the model parameters $\theta_{\delta i}$ 
and an open circle shows the posterior mean of the model standard deviation $\sigma_{\delta}$. At the first step, with the complete 11-parameter model, the posterior mean of $\sigma_{\delta}$ is 0.396 and the parameter with the largest $\operatorname{COV}(=2.31)$ is $\theta_{\delta 8}$. Therefore, the term $\theta_{\delta 8} h_{\delta 8}$ is dropped to simplify the model. Then the reduced model is assessed and the next unnecessary term is removed. After 5 steps, the largest $\operatorname{COV}$ (for parameter $\theta_{\delta 7}$ ) is found to be close in magnitude to $\sigma_{\delta}$ and a further reduction (from Step 5 to Step 6) deteriorates the quality of the model (i.e., $\sigma_{\delta}$ increases significantly.) Stopping at this step, the model is left with seven terms.

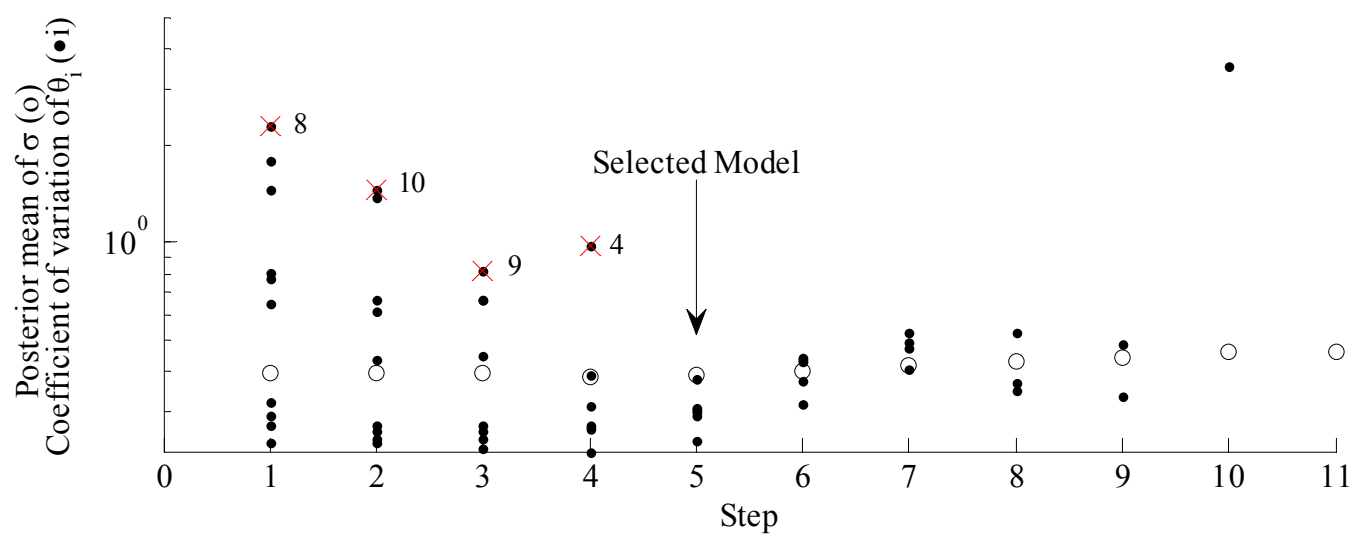

Figure 3-4. Stepwise deletion process for deformation demand model, where $(\times)$ indicates term to be removed

Eq. (3-9) shows the proposed probabilistic model for deformation demand,

$$
\begin{aligned}
D_{\delta}\left(\mathbf{x}, \mathbf{w}, \boldsymbol{\Theta}_{\delta}\right)= & \hat{d}_{\delta}(\mathbf{x}, \mathbf{w})+\theta_{\delta 1}+\theta_{\delta 2} \hat{d}_{\delta}(\mathbf{x}, \mathbf{w})+\theta_{\delta 3} \ln \left(\frac{W_{s} \cdot T_{n}}{H_{H}}\right)+\theta_{\delta 5} \ln \left(\frac{H_{s}}{H_{H}}\right) \\
& +\theta_{\delta 6} \ln \left(\frac{T_{p}}{T_{n}}\right)+\theta_{\delta 7} \ln \left(\frac{R D}{H_{H}}\right)+\theta_{\delta 11} \ln \left(\frac{k_{t}}{k_{f}}\right)+\sigma_{\delta} \varepsilon_{\delta}
\end{aligned}
$$


Assessing all possible subsets of explanatory functions that have seven terms, excluding the lower bound data, the selected model shown in Eq. (3-9) satisfies the selection criteria of the adjusted $\mathrm{R}^{2}$ (Theil 1961), Mallows' $\mathrm{C}_{\mathrm{p}}$ (Mallows 1973) and Corrected Akaike's Informatin Criterion (AICc) (Hurvich and Tsai 1989) better than other models.

A non-informative prior is used in the Bayesian approach because no prior information is available on the unknown parameters $\boldsymbol{\Theta}_{\delta}=\left(\boldsymbol{\theta}_{\delta}, \sigma_{\delta}\right)$ before conducting the virtual experiments. Following Box and Tiao (1992), $p\left(\boldsymbol{\theta}_{\delta}, \sigma_{\delta}\right) \propto \sigma_{\delta}^{-1}$ is selected for the non-informative prior. Table 3-3 presents the posterior statistics of the model parameters in Eq. (3-9.) Figure 3-5 shows plots of predicted versus measured demands based on the deterministic (left) and probabilistic (right) models. For the probabilistic model the median predictions are shown. The equality data are shown as solid dots and the lower bound data are shown as open triangles. The dashed lines in the Fig. 3-5(b) delimit the region within one standard deviation of the model.

Table 3-3. Posterior statistics of the parameters in the deformation demand model

\begin{tabular}{ccccccccccc}
\hline & & \multirow{8}{*}{ Standard } & \multicolumn{8}{c}{ Correlation coefficient } \\
\cline { 7 - 11 } Parameter & Mean & Deviation & $\theta_{\delta 1}$ & $\theta_{\delta 2}$ & $\theta_{\delta 3}$ & $\theta_{\delta 5}$ & $\theta_{\delta 6}$ & $\theta_{\delta 7}$ & $\theta_{\delta 11}$ & $\sigma_{\delta}$ \\
\hline$\theta_{\delta 1}$ & 2.28 & 0.709 & 1 & & & & & & & \\
$\theta_{\delta 2}$ & -0.27 & 0.083 & 0.29 & 1 & & & & & & \\
$\theta_{\delta 3}$ & 0.329 & 0.097 & -0.21 & -0.65 & 1 & & & & & \\
$\theta_{\delta 5}$ & 0.392 & 0.119 & 0.52 & -0.52 & 0.26 & 1 & & & & \\
$\theta_{\delta 6}$ & -0.77 & 0.188 & -0.44 & 0.58 & -0.21 & -0.82 & 1 & & & \\
$\theta_{\delta 7}$ & -0.53 & 0.198 & -0.40 & 0.12 & -0.23 & -0.38 & 0.503 & 1 & & \\
$\theta_{\delta 11}$ & 0.194 & 0.058 & 0.85 & 0.18 & -0.09 & 0.26 & -0.31 & -0.35 & 1 & \\
$\sigma_{\delta}$ & 0.387 & 0.034 & -0.07 & -0.07 & 0.09 & 0.03 & -0.05 & -0.03 & -0.11 & 1 \\
\hline
\end{tabular}


In a perfect demand model, all the solid dots should be lined up along the 1:1 line and all the open triangles should be above the 1:1 line. However, Figure 3-5(a) clearly shows that the deterministic model is biased on the non-conservative side, whereas the proposed probabilistic demand model corrects the bias as shown in Figure 3-5(b.)

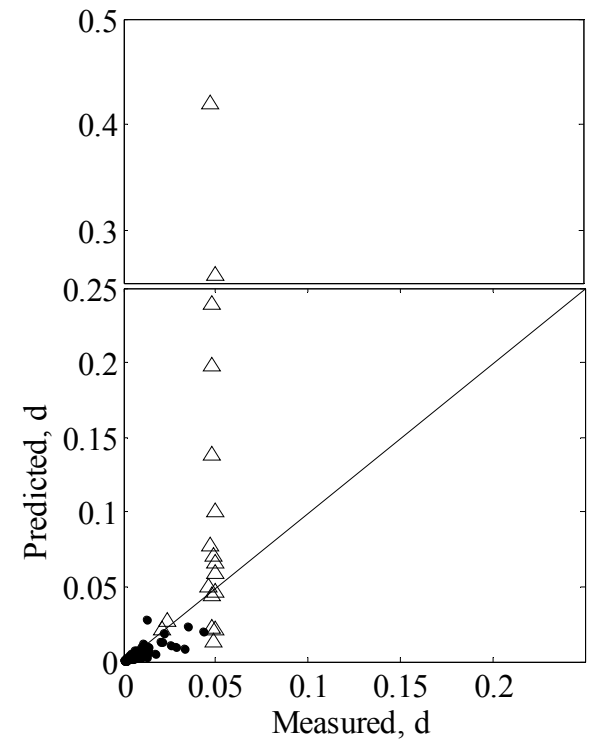

(a)

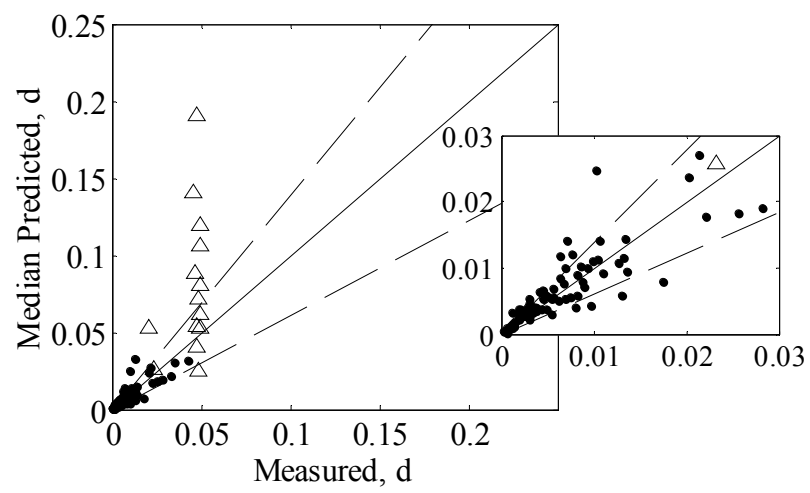

(b)

Figure 3-5. Comparison between measured versus predicted deformation demands, (a) deterministic model, (b) median probabilistic model

\subsubsection{Shear Demand Model}

Figure 3-6 summarizes the stepwise deletion process for the shear demand model. As in Figure 3-4, the solid dots show the posterior COV of the model parameters $\theta_{v i}$ and open circle shows the posterior mean of the model standard deviation $\sigma_{v}$ at each step. After eight steps, further reduction of the correction terms causes the posterior mean of $\sigma_{v}$ to increase by an unacceptable amount. Stopping at this step, the shear demand model is left with the four terms shown in Eq. (3-10.) 


$$
D_{v}\left(\mathbf{x}, \mathbf{w}, \boldsymbol{\Theta}_{v}\right)=\hat{d}_{v}(\mathbf{x}, \mathbf{w})+\theta_{v 1}+\theta_{v 2} \hat{d}_{v}(\mathbf{x}, \mathbf{w})+\theta_{v 8} \ln \left(\frac{C_{s}}{C_{s \max }}\right)+\theta_{v 11} \ln \left(\frac{k_{t}}{k_{f}}\right)+\sigma_{v} \varepsilon_{v}
$$

Again, all possible subsets of explanatory functions for shear demand model having four terms are checked for the criteria of adjusted $\mathrm{R}^{2}$, Mallows' $\mathrm{C}_{\mathrm{p}}$ and AICc and the results shows that they are in agreement with the results from stepwise deletion method.

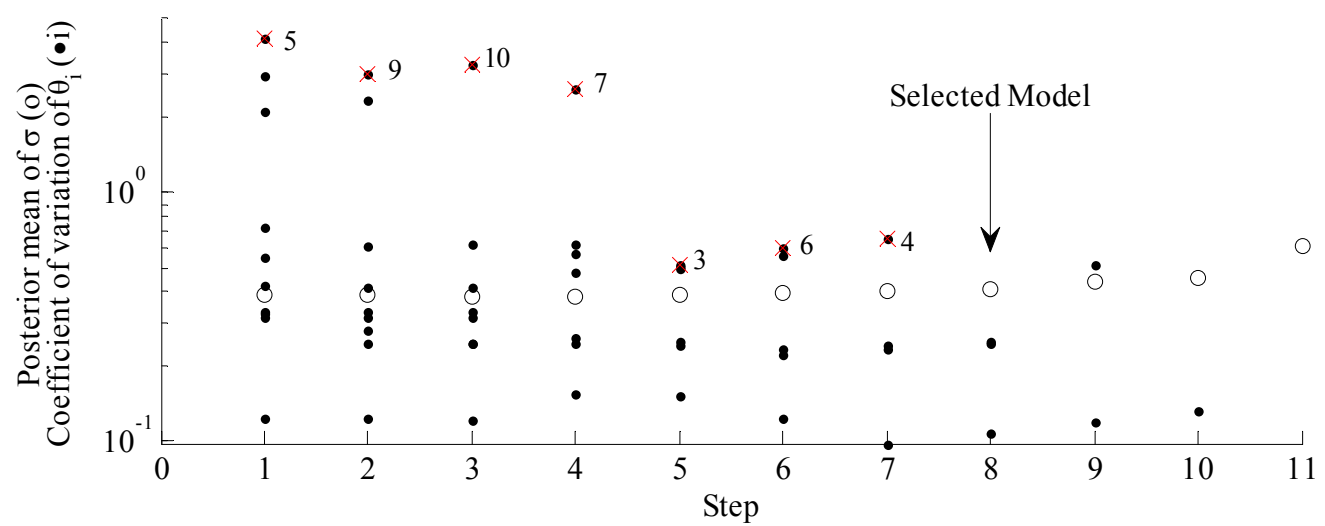

Figure 3-6. Stepwise deletion process for shear demand model, where $(\times)$ indicates term to be removed

The model is formulated as the natural logarithm of the shear demand at the base of the tower normalized by the mean value of the yield shear force defined as $\hat{V}_{y}=\hat{f}_{y} A_{t}(3 / 4)\left(r_{t o}^{2}+r_{t i}^{2}\right) /\left(r_{t o}^{2}+r_{t o} r_{t i}+r_{t i}^{2}\right)$, where $\hat{f}_{y}=$ expected yield stress of steel, $A=$ tower base cross section area, and $r_{t o}$ and $r_{t i}=$ outer and inner diameter of the tower section, respectively. As in developing the deformation demand model, due to the lack of prior information on the unknown model parameters $\boldsymbol{\Theta}_{v}=\left(\boldsymbol{\theta}_{v}, \sigma_{v}\right)$, a non-informative prior is used in assessing the posterior statistics. Table 3-4 gives the posterior statistics 
for shear demand model parameters. Figure 3-7 shows plots of predicted versus measured shear demands based on the deterministic (left) and probabilistic (right) models. Comments analogous to those made for deformation demand model can be made also for the shear demand model based on Figure 3-7. Whereas, the deterministic shear demand model is clearly biased on the non-conservative side, the proposed probabilistic model properly corrects for the bias and gives unbiased predictions of the demands.

Table 3-4. Posterior statistics of the parameters in the shear demand model

\begin{tabular}{cccccccc}
\hline & & Standard & \multicolumn{5}{c}{ Correlation coefficient } \\
\cline { 4 - 8 } Parameter & Mean & Deviation & $\theta_{v 1}$ & $\theta_{v 2}$ & $\theta_{v 8}$ & $\theta_{v 11}$ & $\sigma_{v}$ \\
\hline$\theta_{v 1}$ & -3.65 & 0.693 & 1 & & & & \\
$\theta_{v 2}$ & -0.49 & 0.053 & 0.75 & 1 & & & \\
$\theta_{v 8}$ & -0.73 & 0.180 & 0.67 & 0.32 & 1 & & \\
$\theta_{v 11}$ & -0.26 & 0.066 & 0.89 & 0.40 & 0.65 & 1 & \\
$\sigma_{v}$ & 0.412 & 0.035 & 0.04 & 0.17 & -0.02 & -0.06 & 1 \\
\hline
\end{tabular}

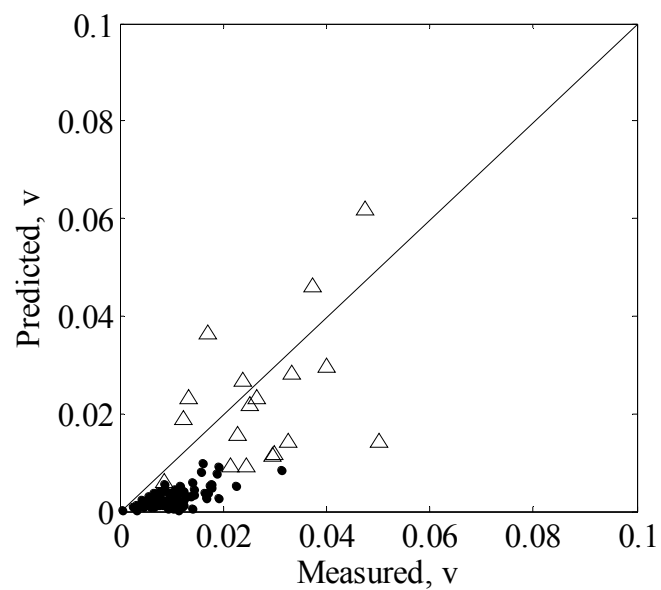

(a)

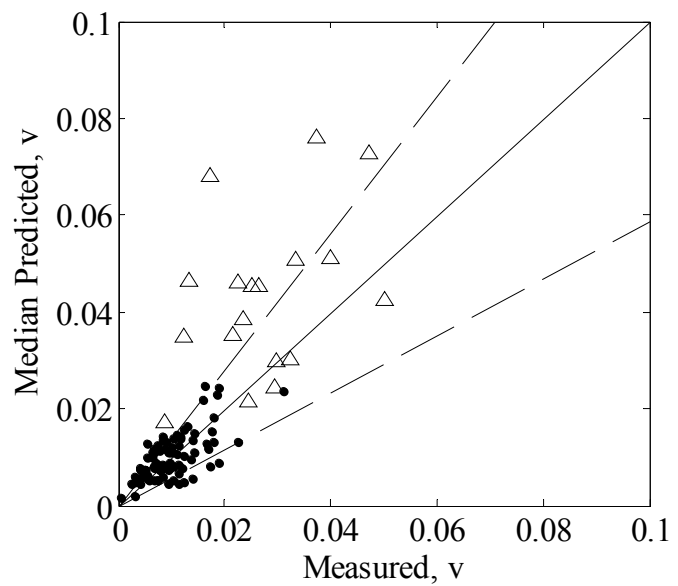

(b)

Figure 3-7. Comparison between measured versus predicted shear demands, (a) deterministic model, (b) median probabilistic model 


\subsubsection{Moment Demand Model}

The probabilistic demand model for moment is formulated as the natural logarithm of the moment demand at the tower base normalized by $\hat{M}_{y}=\hat{f}_{y} S_{t}$, where $S_{t}=$ elastic section modulus at the tower base. As for the deformation and shear demand models, a stepwise deletion process is used to detect unnecessary explanatory functions that can be dropped to simplify the probabilistic model. Figure 3-8 summarizes the stepwise deletion process for the moment demand model, where solid dots and open circle representing the posterior COV of the model parameters $\theta_{m i}$ and the posterior mean of the model standard deviation $\sigma_{m}$ at each step, respectively. Stopping after nine steps, the selected model shown in Eq. (3-11) has three explanatory functions in the correction term. This selection is confirmed again by the values of the adjusted $R^{2}$, Mallows' $C_{p}$ and AICc. The model selection results in the following form for the probabilistic moment demand model:

$$
D_{m}\left(\mathbf{x}, \mathbf{w}, \boldsymbol{\Theta}_{m}\right)=\hat{d}_{m}(\mathbf{x}, \mathbf{w})+\theta_{m 1}+\theta_{m 2} \hat{d}_{m}(\mathbf{x}, \mathbf{w})+\theta_{m 3} \ln \left(\frac{W_{s} \cdot T_{n}}{H_{H}}\right)+\sigma_{m} \varepsilon_{m}
$$

As in developing the deformation and shear demand model, due a lack of prior information on model parameters, a non-informative prior is used to estimate $\boldsymbol{\Theta}_{m}=\left(\boldsymbol{\theta}_{m}, \sigma_{m}\right)$. Table 3-5 gives the posterior statistics of model parameters and Figure 39 shows plots of predicted versus measured moment demands based on the deterministic (left) and probabilistic (right) models. Similar comments to those made for Figures 3-5 and 3-7 can be made for Figure 3-9. 


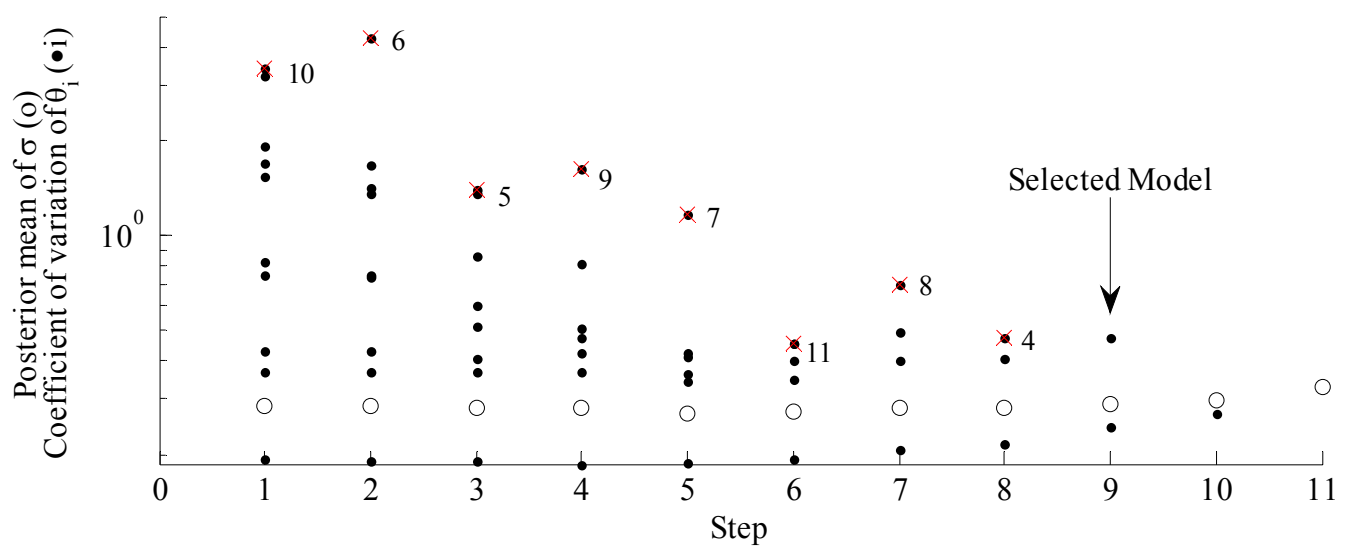

Figure 3-8. Stepwise deletion process for moment demand model, where $(\times)$ indicates term to be removed

Table 3-5. Posterior statistics of the parameters in the moment demand model

\begin{tabular}{lllllll}
\hline & & \multirow{2}{*}{$\begin{array}{l}\text { Standard } \\
\text { Parameter }\end{array}$} & \multicolumn{5}{c}{ Correlation coefficient } \\
\cline { 5 - 7 } \cline { 5 - 7 } & Mean & Deviation & $\theta_{m 1}$ & $\theta_{m 2}$ & $\theta_{m 3}$ & $\sigma_{m}$ \\
\hline$\theta_{m 1}$ & 0.572 & 0.087 & 1 & & & \\
$\theta_{m 2}$ & -0.19 & 0.048 & 0.87 & 1 & & \\
$\theta_{m 3}$ & 0.141 & 0.066 & -0.42 & -0.74 & 1 & \\
$\sigma_{m}$ & 0.291 & 0.023 & 0.12 & 0.07 & 0.03 & 1 \\
\hline
\end{tabular}

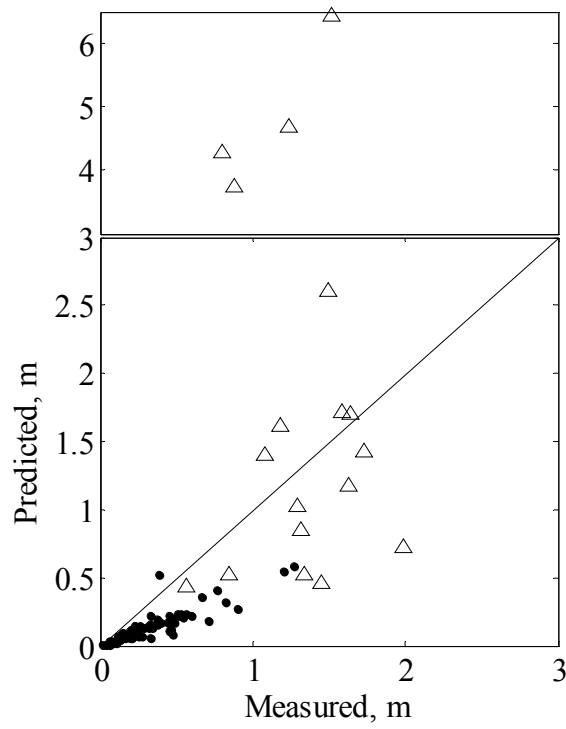

(a)

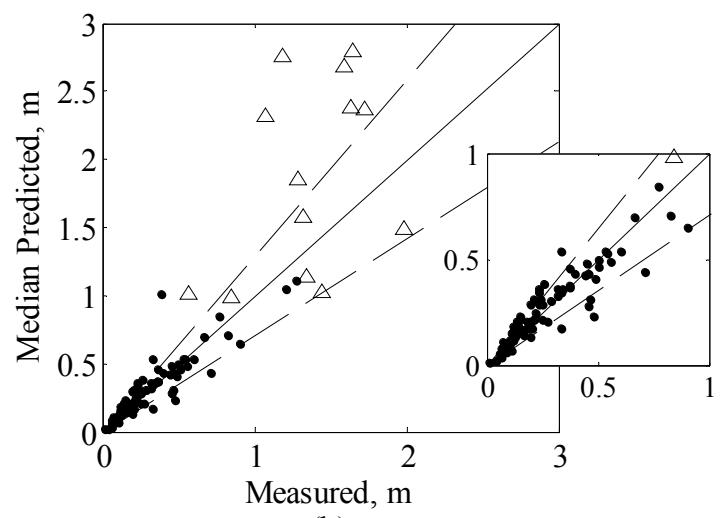

(b)

Figure 3-9. Comparison between measured versus predicted moment demands, (a) deterministic model, (b) median probabilistic model 


\subsection{Fragility Estimates of an Example Offshore Wind Turbine Support Structure}

Fragility is defined as the conditional probability of not meeting specified capacity levels for given value of the vector w . Following Gardoni et al. (2002), a predictive estimate of the fragility is formulated as

$$
\tilde{F}_{j}(\mathbf{w})=P\left[\bigcup_{k}\left\{g_{k j}\left(\mathbf{x}, \mathbf{w}, \boldsymbol{\Theta}_{k}\right) \leq 0\right\} \mid \mathbf{w}\right]
$$

where $g_{k j}\left(\mathbf{x}, \mathbf{w}, \boldsymbol{\Theta}_{k}\right)$ is the $k^{\text {th }}$ limit state function defined as

$$
g_{k j}\left(\mathbf{x}, \mathbf{w}, \boldsymbol{\Theta}_{k}\right)=C_{k j}(\mathbf{x})-D_{k}\left(\mathbf{x}, \mathbf{w}, \boldsymbol{\Theta}_{k}\right)
$$

in which $C_{k j}(\mathbf{x})$ represent the capacity corresponding to $D_{k}\left(\mathbf{x}, \mathbf{w}, \boldsymbol{\Theta}_{k}\right)$, and $j=s, y$ or $u$ identifies the service, yield and ultimate capacity levels, respectively.

In this section, the fragility estimates are developed for the performance levels that delimit four possible damage states of a support structure. Table 3-6 shows the description of the considered damage states, the limiting performance levels, and the corresponding capacities.

This subsection focuses on the assessment of the fragility of a typical 5-MW offshore wind turbine support structure installed in a $20 \mathrm{~m}$ water depth and subject to day-to-day environmental and operational loadings. The considered wind turbine is assumed to be supported by a mono-pile foundation that is typical for this water depth. The specifications of the configuration of interest can be found in Jonkman et al. (2009.) Also, Table 3-7 shows a summary of the properties of considered wind turbine support structure. 
Table 3-6. Proposed damage states and corresponding limit states and natural hazards

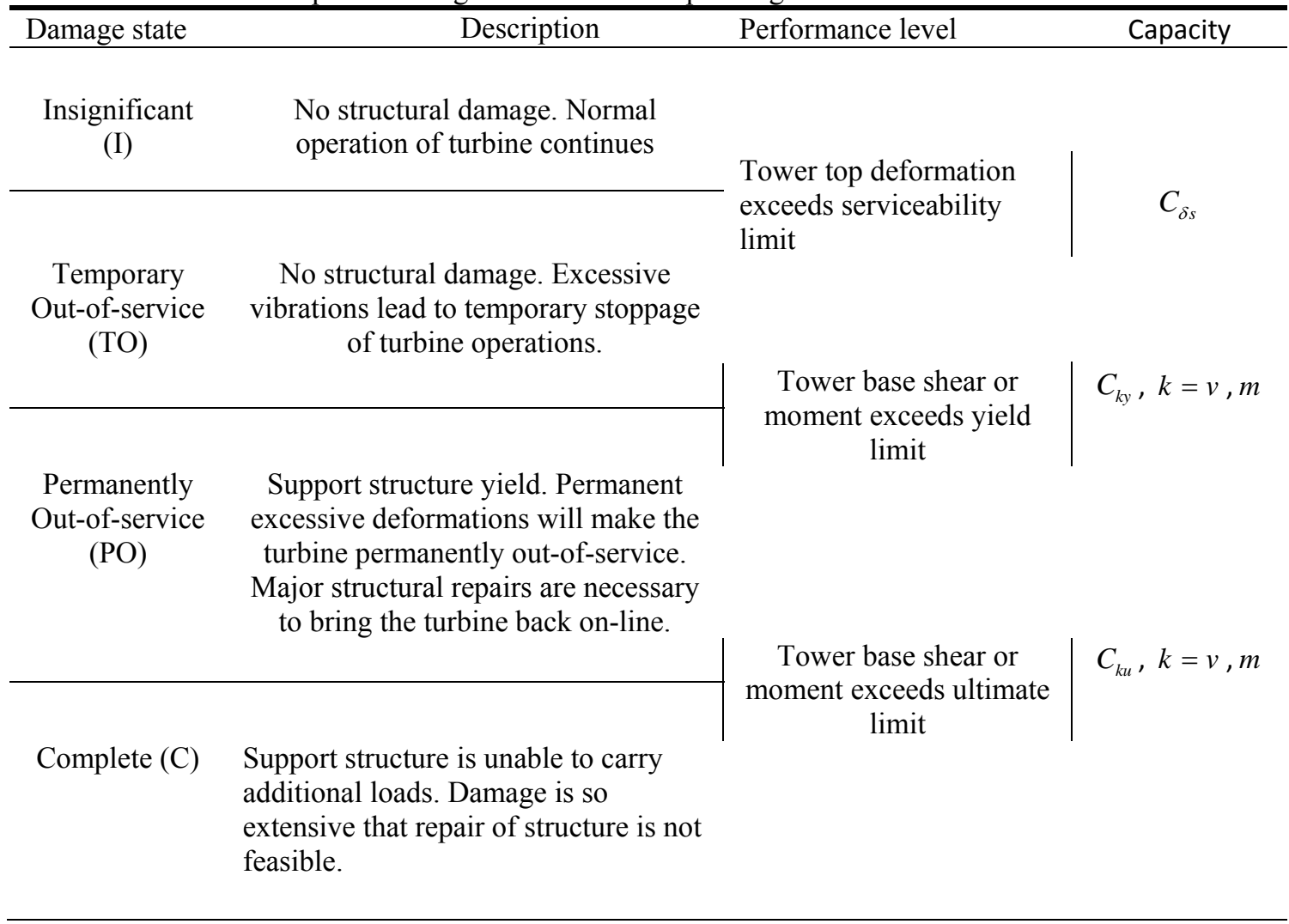

As shown in Table 3-6, three modes of failure are considered: drift, shear and bending. Following Lavassas et al. (2003), a drift of $0.5 \%$ is considered as deformation capacity, $C_{\delta s}$, that is used to define the serviceability limit. The shear capacity is defined as $C_{v j}=f_{j} A_{t}(3 / 4)\left(r_{t o}{ }^{2}+r_{t i}{ }^{2}\right) /\left(r_{t o}{ }^{2}+r_{t o} r_{t i}+r_{t i}{ }^{2}\right)$, where $f_{j}$ is equal to the yield stress, $f_{y}$, or the ultimate stress, $f_{u}$, for the yield and ultimate capacity, respectively. In developing the fragility estimates, $f_{y}$ and $f_{u}$ are considered to be lognormal random variables, respectively with the means of 300 and $410 \mathrm{MPa}$ (for a structural steel of Grade S235 according to EN 10025 standard (CEN 2004)), and a coefficient of variation of $10 \%$. 
The yield bending moment capacity is computed as $C_{m y}=f_{y} S_{t}$. Finally, the ultimate bending moment capacity, $C_{m u}$, is considered to be lognormal random variable with a mean of 390.6 MN-m and a standard deviation of $39.57 \mathrm{MN}-\mathrm{m}$. The statistics of $C_{m u}$ is obtained using moment-curvature diagrams constructed for the tubular cross section of the tower base, considering the stress-strain curve of structural steel of Grade S235.

Table 3-7. Properties of the NREL offshore 5-MW baseline wind turbine

\begin{tabular}{ll}
\hline Property & Value \\
\hline Rating & $5 \mathrm{MW}$ \\
Rotor diameter & $126 \mathrm{~m}$ \\
Hub height & $90 \mathrm{~m}$ \\
Cut-in, rated, cut-out wind speed & $3 \mathrm{~m} / \mathrm{s}, 11.4 \mathrm{~m} / \mathrm{s}, 25 \mathrm{~m} / \mathrm{s}$ \\
Rotor mass & $110,000 \mathrm{~kg}$ \\
Nacelle mass & $240,000 \mathrm{~kg}$ \\
Tower mass & $347,460 \mathrm{~kg}$ \\
Tower top diameter and wall thickness & $3.87 \mathrm{~m}, 0.019 \mathrm{~m}$ \\
Tower base diameter and wall thickness & $6.00 \mathrm{~m}, 0.027 \mathrm{~m}$ \\
\hline
\end{tabular}

Monte Carlo simulations are used to estimate the fragility of the support structure for each failure mode. All the model parameters and the error terms in demand models are considered as random variables with the normal distribution assumed for the model parameters, $\boldsymbol{\theta}_{k}$, and the lognormal distribution assumed for $\sigma_{k}$.

Figure 3-10 shows the predictive fragility estimates for the example offshore wind turbine plotted as a function of $W_{s}$ varying within the turbine operational range and for a significant wave height $H_{s}=1 \mathrm{~m}$. The turbine operational range is defined as the range of the wind speed in which the turbine is operating and producing power with a 
lower limit of cut-in wind speed and an upper limit of cut-out wind speed. The rated wind speed is the wind speed at which a control system is activated to limit the aerodynamic forces on the blades of the wind turbine and keep the power generated constant by changing the blade pitch angle.

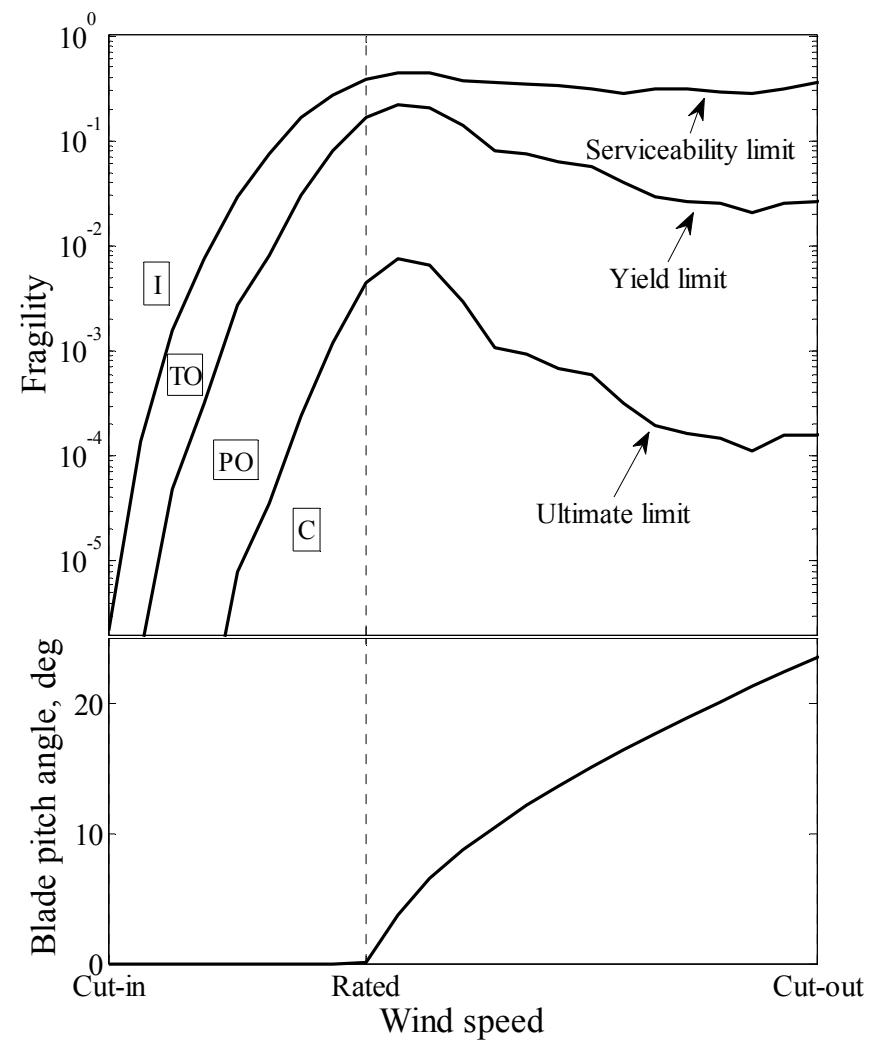

Figure 3-10. Fragility estimates for an offshore wind turbine as a function of mean wind speed for $H_{\mathrm{s}}=1 \mathrm{~m}$

The three curves in the top plot in Figure 3-10 show the fragilities associated to the serviceability, yield and ultimate limits. The reduction in the probabilities of failure after the rated wind speed is due to the activation of the control system at the rated wind speed that changes the blade pitch angle, as shown in the bottom plot. It is also noted 
that for the yield and the ultimate limits, the contribution to the fragility from the bending mode controls and the contribution from the shear mode is negligible. This is as expected for slender elements like the tower of wind turbines.

To study the effect of the variability in $H_{s}$ on the fragility estimates, Figure 3-11 shows the yield bending fragility estimates plotted as a function of $H_{s}$, for different values of $W_{s}$. As shown in the figure, the fragility at the rated wind speed is higher than the fragilities at the other two wind speeds due to the higher wind speed than the cut-in wind speed and operational loading than at the cut-out wind speed. Figure 3-11 also shows that the changes in wave height do not affect noticeably the probability of failure, especially for large wind speeds.

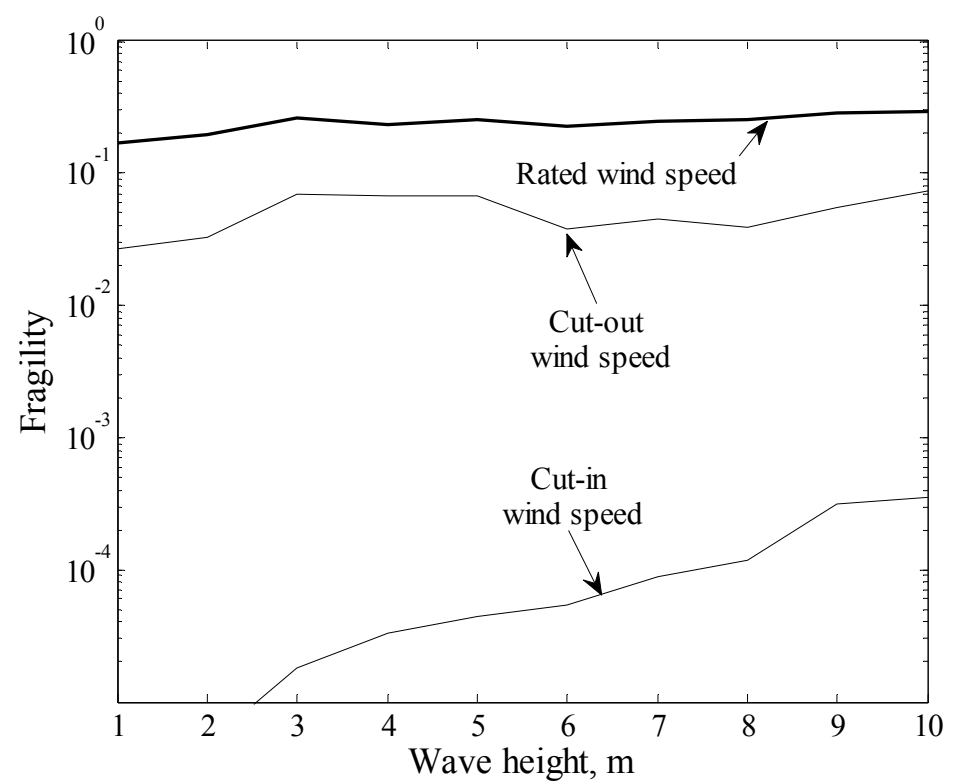

Figure 3-11. Yield bending fragility estimates for an offshore wind turbine as a function of significant wave height at different wind speeds 


\subsection{Conclusions}

This section developed probabilistic models for deformation, shear and moment demands on the support structure of offshore wind turbines. In order to promote their use in practice, the probabilistic models were constructed by adding a correction term to existing deterministic models commonly used for the design of wind turbines. The correction terms were assessed using data obtained from detailed 3D nonlinear FE analyses of wind turbine systems that accounted for the effects of the dynamics soilstructure interaction. A stepwise selection process was used to develop parsimonious model forms and a Bayesian approach was used to assess unknown model parameters. The developed probabilistic models account for the relevant aleatory and epistemic uncertainties in predicting the demand quantities of interest.

The proposed probabilistic demand models were then used to assess the fragility of an example off-shore wind turbine subject to day-to-day wind, wave and current loading. The conditional probabilities of exceeding three specified performance levels (serviceability, yield, and ultimate) were found to increase with the average wind speed up to the rated wind speed. Upon reaching the rated wind speed, a control system is activated to limit the aerodynamic forces on the blades of the wind turbine and keep the power generated constant by changing the blade pitch angle. The activation of the control system was found to reduce the values of the fragilities. The bending mode was found to control the probability of exceeding the yield and ultimate limit states, while the shear failure mode was found to provide negligible contributions to the fragility. Also, 
the fragility estimates show that wave loading does not noticeably affect the probability of failure, especially for large wind speeds. 


\section{PROBABILISTIC SEISMIC DEMAND MODELS AND FRAGILITY ESTIMATES FOR OFFSHORE WIND TURBINES}

\subsection{Introduction}

Previous section assessed the deformation, shear and moment demands on the support structure of offshore wind turbines subject to wind, wave, current and turbine operational loadings properly accounting for the nonlinear soil-structure interaction. A continuous modeling of the pile and the surrounding soil was developed using 3D finite elements that accounts for the nonlinear behavior of the soil and of the soil-pile interface. Because a complete nonlinear 3D finite element analysis can be quite expensive and time consuming, simplified probabilistic demand models were proposed for the deformation, shear and moment demands on the support structure of offshore wind turbines. The probabilistic models were calibrated using the results from the nonlinear 3D finite element analyses and properly accounted for the inherent uncertainties, including the statistical uncertainty (associated with the finite sample size) and the modeling errors (associate with the selection of the variables in the models and the model forms.)

However, with the extensive installation of wind farms in moderate and high seismic regions in the United States and other countries, a new concern has raised about the safety of wind turbine support structures subject to seismic loads (Prowell and Veers 2009.) Early publications on the analysis of dynamic response of wind turbines during earthquake (Bazeos et al. 2002, Lavassas et al. 2003) were based on the simplified 
models that lumped the nacelle and rotor as a point mass at the top of the tower. More refined models developed later to incorporate the aeroelastic interaction into the response analysis (Witcher 2005, Ishihara and Sarwar 2008, Prowell et al. 2010.) Witcher (2005) studied the seismic response of wind turbine support structures using the seismic module within GH Bladed and indicated the importance of time domain simulations to account for aeroelastic interaction. Developing a nonlinear FEM code (CAsT), Ishihara and Sarwar (2008) carried out a time domain analysis of dynamic response of wind turbines to include the tower-rotor coupling. The result was used to introduce a safety factor to the semi-theoretical design formula (based on building design code) in order to modify it to be used for wind turbines. Prowell et al. (2010) calibrated the aeroelastic interaction modeled in FAST using experimental data from a shake-table test of a small onshore $65-\mathrm{kW}$ wind turbine (Prowell et al. 2008.) Yet, all these studies fail to incorporate the dynamic soilstructure interaction.

This section develops novel shear and moment demand models for the support structure of offshore wind turbines subject to seismic loading in addition to wind, wave, current and turbine operational loadings. The approach is consistent with the one used in previous section for wind turbines operating under day-to-day environmental loads and emphasis is given to the additional seismic load. The wind turbine is considered to be operating throughout the earthquake and the aeroelastic interaction is included in the dynamic response analyses of wind turbine support structures. Regarding the generation of deterministic data, the open source aeroelastic simulator, FAST, is modified to incorporate seismic ground motions in the simulations. Finally, fragility estimates are 
presented for an example offshore wind turbine support structure for given values of the intensity measure of the loading (i.e., the mean wind speed and spectral acceleration.)

\subsection{Probabilistic Demand Models}

Following an approach consistent with the one presented in Subsection 3.3, to incorporate the rules of physics and mechanics and facilitate the acceptance of the proposed models, probabilistic demand models are developed by adding a correction term to selected existing deterministic demand models as shown in Eq. (3-5.) Again, probabilistic shear and moment demand models are developed for horizontal axis offshore wind turbines rated between 0.5 and 5 megawatts (medium to large wind turbines.) The wind turbines of interest in this section are supported by a tubular steel tower, which is seated on a steel mono-pile foundation at the base and installed in water depths less than 30 meters. This section predicts the shear and moment demands on the support structures subject to seismic excitation, in addition to wind, wave, current and turbine operational loadings.

\subsubsection{Deterministic Demand Model}

An ideal deterministic model $\hat{d}_{k}(\mathbf{x}, \mathbf{w})$ should be simple and yet accurate, and commonly accepted in practice. Because of these reasons and also to be consistent throughout the dissertation, FAST is used to compute deterministic predictions of the shear and moment demands on the support structure of wind turbines subject to earthquake in addition to wind, wave, current and turbine operational loadings. The currently in practice version 
of FAST does not include the seismic module. However, the recent updates to FAST (Jonkman 2007) that allow a force and moment to be applied at the tower base platform with six degrees of freedom at each time step, provide the possibility to model the earthquake ground motions in a time marching simulation.

Similar to the approach described in the previous section, for given values of the mean wind speed and turbulence intensity, a time history of wind speed is generated by TurbSim and used as an input for the dynamic analysis in FAST. As it is supported by TurbSim, IEC Kaimal model (Kaimal et al. 1972) is used in this study. Linear irregular waves for given significant wave height and wave peak period are modeled using the JONSWAP/Pierson-Moskowitz spectrum (Dean and Dalrymple 1991), also supported by FAST. FAST then uses the Morison's equation to determine the hydrodynamic forces on the tower. Current loading is also incorporated in the Morison's Equation.

For given intensity and duration parameters of the ground motion and frequency content of a filter, synthetic ground motions are generated following Rezaeian and Der Kiureghian (2010.) The generated ground motions are then used as inputs for dynamic analyses also carried out using FAST. Rezaeian and Der Kiureghian (2010) formulated the ground motion process with a stochastic model as

$$
\hat{y}(t)=q(t, \boldsymbol{\alpha})\left[\Psi(t)^{\mathrm{T}} \boldsymbol{\Lambda}\right] \quad t_{m} \leq t<t_{m+1}
$$

where $t$ stands for the time, $q(t, \boldsymbol{\alpha})=$ a modulating function that controls the timevarying intensity of the process, $\Psi(t)=\left[\psi_{1}(t), \ldots, \psi_{n}(t)\right]^{\mathrm{T}}=\mathrm{a}$ unit vector of the deterministic basis functions that controls the evolving frequency content of the process 
and $\boldsymbol{\Lambda}=\left[\Lambda_{1}, \ldots, \Lambda_{j}\right]^{\mathrm{T}}=$ a vector of standard normal random variable that provides the randomness that exists in real ground motions. Following Rezaeian and Der Kiureghian (2010), the gamma modulating function is selected and formulated as

$$
\begin{aligned}
& q(t, \boldsymbol{\alpha})=0 \quad \text { if } t \leq T_{0} \\
& =\alpha_{1}\left(t-T_{0}\right)^{\alpha_{2}-1} \exp \left[-\alpha_{3}\left(t-T_{0}\right)\right] \quad \text { if } \quad T_{0} \leq t
\end{aligned}
$$

This model has four parameters $\boldsymbol{\alpha}=\left(\alpha_{1}, \alpha_{2}, \alpha_{3}, T_{0}\right)$, where $\alpha_{1}, \alpha_{3}>0, \alpha_{2}>1$, and $T_{0}$ denotes the start time of the process. The deterministic basis function is written as a function of the filter parameters, $\boldsymbol{\Phi}\left(t_{j}\right)$, as follows

$$
\psi_{j}\left[t, \boldsymbol{\Phi}\left(t_{j}\right)\right]=\frac{h_{s}\left[t-t_{j}, \boldsymbol{\Phi}\left(t_{j}\right)\right]}{\sqrt{\sum_{i=1}^{n} h_{s}^{2}\left[t-t_{i}, \boldsymbol{\Phi}\left(t_{i}\right)\right]}} \quad t_{n} \leq t<t_{n+1} ; 1 \leq j \leq n
$$

where $h_{s}[t-\tau, \boldsymbol{\Phi}(\tau)]$ represents the pseudo-acceleration response of a single-degree-offreedom linear oscillator subject to a unit impulse and is formulated as

$$
\begin{aligned}
h_{s}[t-\tau, \boldsymbol{\Phi}(\tau)] & =\frac{\omega_{f}(\tau)}{\sqrt{1-\xi_{f}^{2}(\tau)}} \exp \left[-\xi_{f}(\tau) \omega_{f}(\tau)(t-\tau)\right] \times \sin \left[\omega_{f}(\tau) \sqrt{1-\xi_{f}^{2}(\tau)}(t-\tau)\right] \quad \tau \leq t \\
= & 0 \quad \text { otherwise }
\end{aligned}
$$

where $\tau=$ the time of the pulse, $\omega_{f}(\tau)=$ natural frequency and $\xi_{f}(\tau)=$ damping ratio of the filter.

The unknown parameters of the process $\boldsymbol{\alpha}=\left(\alpha_{1}, \alpha_{2}, \alpha_{3}, T_{0}\right)$ and $\boldsymbol{\Phi}\left(t_{j}\right)=\left[\omega_{f}(\tau)\right.$, $\left.\xi_{f}(\tau)\right]$ are assessed by matching the properties of generated and reference ground motions. The modulating function parameters $\left(\alpha_{1}, \alpha_{2}, \alpha_{3}\right)$ are related to ground motion 
time history variables $\left(\bar{I}_{a}, D_{5-95}, t_{\text {mid }}\right)$, where $\bar{I}_{a}=$ expected Arias intensity (Arias 1970) of the acceleration process, $D_{5-95}=$ time interval between the instants at which the $5 \%$ and $95 \%$ of the expected Arias intensity are reached, and $t_{\text {mid }}=$ time at which $45 \%$ of the expected Arias intensity is reached. The filter parameters $\left[\omega_{f}(\tau), \xi_{f}(\tau)\right]$, which control the evolving predominant frequency and bandwidth of the process, are assessed based on their relations to the rate of zero-level up-crossings and the cumulative number of negative maxima and positive minima of the acceleration process. More details on the assessment of the unknown parameters of this process are presented in Rezaeian and Der Kiureghian (2010.)

In case of a seismic event, a base acceleration time history is responsible for the resulting forces in the structure. FAST does not accept an acceleration time history as an input. Therefore a time history of force, $F_{a}(t)$, is applied to the platform. Using an artificially large mass for the support platform, the force $F_{a}(t)=M a(t)$ produces the desirable acceleration $a(t)$ at the base of the turbine support structure, where $M$ is the total mass of the support platform and the wind turbine.

\subsubsection{Model Correction}

Correction term, $\gamma_{k}\left(\mathbf{x}, \mathbf{w}, \boldsymbol{\theta}_{k}\right)$, is intended to adjust for the bias inherent in the deterministic model. A linear form presented in Eq. (3-6) is used for the correction term, where for each demand of interest $k, \boldsymbol{\theta}_{k}=\left[\theta_{k i}\right]$ and $h_{k i}(\mathbf{x}, \mathbf{w}), i=1, \ldots, p$, are, respectively, unknown model parameters and selected explanatory functions. Also, in 
this section, $k=v$ or $m$, for the shear or moment demand, respectively. Ideally, explanatory functions should be selected from laws of mechanics and structural dynamics. The term $h_{k 1}(\mathbf{x}, \mathbf{w})=1$ is selected to capture potential constant bias in the model that is independent of $\mathbf{x}$ and $\mathbf{w}$, and $h_{k 2}(\mathbf{x}, \mathbf{w})=\hat{d}_{k}(\mathbf{x}, \mathbf{w})$ to capture any possible under- or over-estimation of the deterministic models. To capture possible dependence of residuals on foundation, environment and earthquake parameters, which are not properly included in the deterministic model, additional explanatory functions are also considered. Table 4-1 shows candidate explanatory functions for the demand models. To characterize the influence of wind and wave parameters, $h_{k 3}-h_{k 6}$ are selected as normalized functions of the mean wind speed, turbulence intensity, significant wave height and wave peak period, respectively. The explanatory functions $h_{k 7}-h_{k 13}$ are considered to incorporate the influence of the magnitude and frequency content of the ground motion. In addition, $h_{k 14}$ is considered to capture the possible influence of the rotor diameter. Finally, $h_{k 15}-h_{k 18}$ are considered to capture the possible influence of the foundation stiffness, which is not included in the deterministic model. A Bayesian inference is then used to estimate the unknown model parameters $\boldsymbol{\theta}_{k}$. Due to the lack of available data needed to conduct the statistical analysis required to estimate the model parameters, a database of virtual experiments is generated using detailed nonlinear dynamic analyses of offshore wind turbine support structures as explained next. 
Table 4-1. Explanatory functions for demand models

\begin{tabular}{lll}
\hline Explanatory function & Formula & Parameters \\
\hline$h_{k 1}$ & 1 & $k=v$ or $m$ \\
$h_{k 2}$ & $\hat{d}_{k}$ & $\hat{d}_{k}=$ Deterministic shear or moment demand \\
$h_{k 3}$ & $\ln \left(W_{s} \cdot T_{n} / H_{H}\right)$ & \\
$h_{k 4}$ & $\ln \left(I T_{w}\right)$ & \\
$h_{k 5}$ & $\ln \left(H_{s} / H_{H}\right)$ & \\
$h_{k 6}$ & $\ln \left(T_{p} / T_{n}\right)$ & \\
$h_{k 7}$ & $\ln \left(S_{a} / g\right)$ & $S_{a}=$ Spectral acceleration; $g$ = ground acceleration \\
$h_{k 8}$ & $\ln \left(S_{d} / H_{H}\right)$ & $S_{d}=$ Spectral displacement \\
$h_{k 9}$ & $\ln (P G A / g)$ & $P G A=$ Peak ground acceleration \\
$h_{k 10}$ & $\ln \left(P G V \cdot T_{n} / H_{H}\right)$ & $P G V=$ Peak ground velocity \\
$h_{k 11}$ & $\ln \left(P G D / H_{H}\right)$ & $P G D=$ Peak ground displacement \\
$h_{k 12}$ & $\ln \left[2 \pi P G V /\left(P G A \cdot T_{n}\right)\right]$ & \\
$h_{k 13}$ & $\ln \left[2 \pi P G D /\left(P G V \cdot T_{n}\right)\right]$ & \\
$h_{k 14}$ & $\ln \left(R D / H_{H}\right)$ & \\
$h_{k 15}$ & $\ln \left(C_{s} / C_{\text {smax }}\right)$ & \\
$h_{k 16}$ & $\ln \left(C_{\text {soil }} / E_{\text {soil }}\right)$ & \\
$h_{k 17}$ & $\ln \left[\tan \left(\phi_{\text {soil }}\right)\right]$ & \\
$h_{k 18}$ & $\ln \left(k_{t} / k_{f}\right)$ & \\
\hline
\end{tabular}

\subsubsection{Virtual Experiment Data}

Representative ground motions are assigned to the configurations generated by conducting an experimental design as explained in Section 3. A Latin hypercube sampling technique, which is a space filling technique and maximizes the minimum distance between the sample points, is used to ensure that the sampling has a good coverage of the design space. See Subsection 3.2.1 for more details on the experimental design and the variables and their considered ranges.

Representative ground motions for the virtual experiments must be properly selected in order to assess the seismic demand variables of interest and their associated 
uncertainties. In particular, the selected ground motion records should capture the characteristics of the possible seismic hazards, including their return periods, intensities, frequency contents, and durations (Krawinkler et al. 2003.) Ground motion records are selected from the PEER (Pacific Earthquake Engineering Research Center) NGA database (1999.) Following Shome and Cornell (1999), the selected ground motions are subdivided into five bins based on moment magnitude $\left(M_{e q}\right)$ and the closest distance between the record location and the rupture zone $\left(R_{e q .}\right)$ Table 4-2 shows the bins from which the ground motions are selected. Each bin represents specific combinations of the earthquake characteristics and the collection of all bins captures all possible characteristics. A total of 20 representative ground motion records are selected from each bin.

Table 4-2. Bins from which ground motions are selected

\begin{tabular}{ccccc}
\hline Bin No. & Bin characteristics & Magnitude & Distance $(\mathrm{km})$ & No. of Records \\
\hline 1 & Large magnitude, small distance & $\mathrm{M}_{\mathrm{eq}}>6.5$ & $13<\mathrm{R}_{\mathrm{eq}}<30$ & 20 \\
2 & Large magnitude, large distance & $\mathrm{M}_{\mathrm{eq}}>6.5$ & $\mathrm{R}_{\mathrm{eq}}>30$ & 20 \\
3 & Small magnitude, small distance & $\mathrm{M}_{\mathrm{eq}}<6.5$ & $13<\mathrm{R}_{\mathrm{eq}}<30$ & 20 \\
4 & Small magnitude, large distance & $\mathrm{M}_{\mathrm{eq}}<6.5$ & $\mathrm{R}_{\mathrm{eq}}>30$ & 20 \\
5 & Near fault & $\mathrm{M}_{\mathrm{eq}}>6.5$ & $\mathrm{R}_{\mathrm{eq}}<13$ & 20 \\
\hline
\end{tabular}

Finite element models are developed in ABAQUS to simulate the dynamic response of the support structure of typical offshore wind turbines, subject to wind, wave, current and turbine operational loading as well as earthquake. The finite element model of the support structure is constructed such that it accounts for the nonlinearity of the soil behavior and soil-structure interaction. Again, the aerodynamics of the turbine is 
simulated by the support of FAST. FAST produces the time history of the forces at the top of the tower due to the wind and the operation of the turbine. The time history of wind loading used as an input for FAST is generated using TurbSim. The result of this simulation is the operational loading on the tower, which is used in the finite element model of the support structure as an external loading in addition to wave and current loading. At the same step of analysis, earthquake ground motions are applied at the base of the FE model, assuming the turbine is operating throughout the earthquake. Therefore, a structural damping of $5 \%$ is assigned to the tower to incorporate the aerodynamic damping due to continuous operation of wind turbine during the earthquake (Witcher 2005.)

Finally following an approach consistent with the one used in the previous section, the data from the virtual experiments are divided into equality and lower bound data. A threshold of 5\% is considered for drift, such that if the maximum drift during one time history analysis is less than $5 \%$, then the shear and moment data are considered as equality data. If an analysis produces a drift that exceeds 5\%, then the maximum shear and moment that occurred prior to reaching the $5 \%$ drift are considered as the lower bound data for the shear and moment, respectively.

\subsubsection{Model Selection}

To develop parsimonious probabilistic demand models (i.e., with only the explanatory functions that are strictly needed) a model selection process is used to identify the important explanatory functions among the candidates presented in Table 4- 
1. A stepwise deletion procedure is responsible for the model selection. As explained in Section 3.3.3, starting with a comprehensive candidate form of $\gamma_{k}\left(\mathbf{x}, \mathbf{w}, \boldsymbol{\theta}_{k}\right)$, unnecessary terms are deleted in a stepwise manner based on the posterior statistics of model parameters. At each step, the term $h_{k i}$ whose coefficient $\theta_{k i}$ has the largest posterior coefficient of variation (COV) is deleted. Model reduction is continued until an unacceptable amount of growth is seen in the value of $\sigma_{v}$.

Figure 4-1 summarizes the stepwise deletion process for seismic shear demand model. At each step, solid dots show the posterior COVs of the model parameters $\theta_{v i}$ and open circle shows the posterior mean of the standard deviation of the model $\sigma_{v}$. It is seen that after fifteen steps, further model reduction will deteriorate the accuracy of the model (i.e., $\sigma_{v}$ increases significantly.) Stopping at this step, the model is left with four terms.

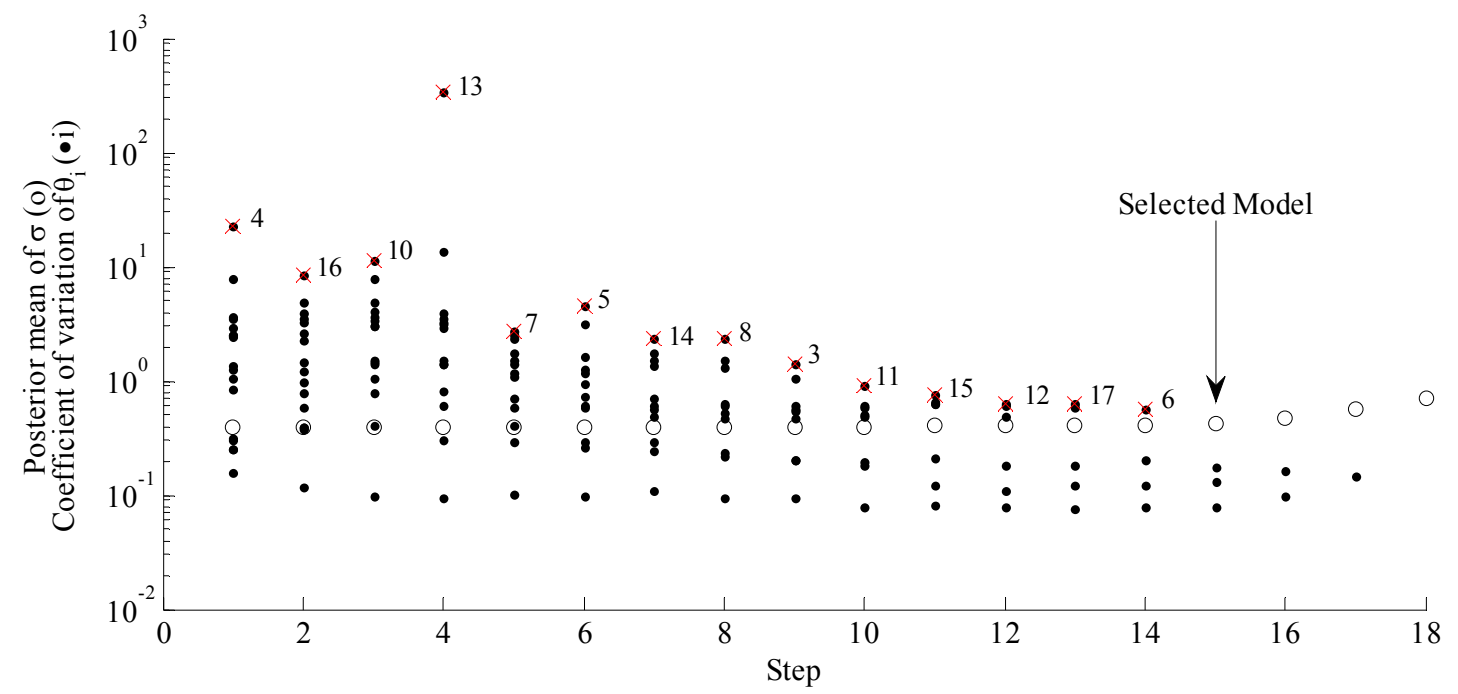

Figure 4-1. Stepwise deletion process for seismic shear demand model, where $(\times)$ indicates term to be removed 
Upon carrying out the model selection process, final probabilistic seismic shear demand model is written as:

$$
D_{v}\left(\mathbf{x}, \mathbf{w}, \boldsymbol{\Theta}_{v}\right)=\hat{d}_{v}(\mathbf{x}, \mathbf{w})+\theta_{v 1}+\theta_{v 2} \hat{d}_{v}(\mathbf{x}, \mathbf{w})+\theta_{v 9} \ln \left(\frac{P G A}{g}\right)+\theta_{v 18} \ln \left(\frac{k_{t}}{k_{f}}\right)+\sigma_{v} \varepsilon_{v}
$$

It is noteworthy that excluding the lower bound data, the selected model in Eq. (4-5) also satisfies the model selection criteria of the adjusted $R^{2}$, Mallows' $C_{p}$ and Corrected Akaike's Information Criterion (AICc) better than other possible subsets of explanatory functions with four terms.

Likewise, the model selection process for seismic moment model is carried out. The result of the stepwise deletion process is shown in Figure 4-2. As in Figure 4-1, solid dots show the posterior of the model parameters $\theta_{m i}$ and open circle shows the posterior mean of the model standard deviation $\sigma_{m}$ at each step.

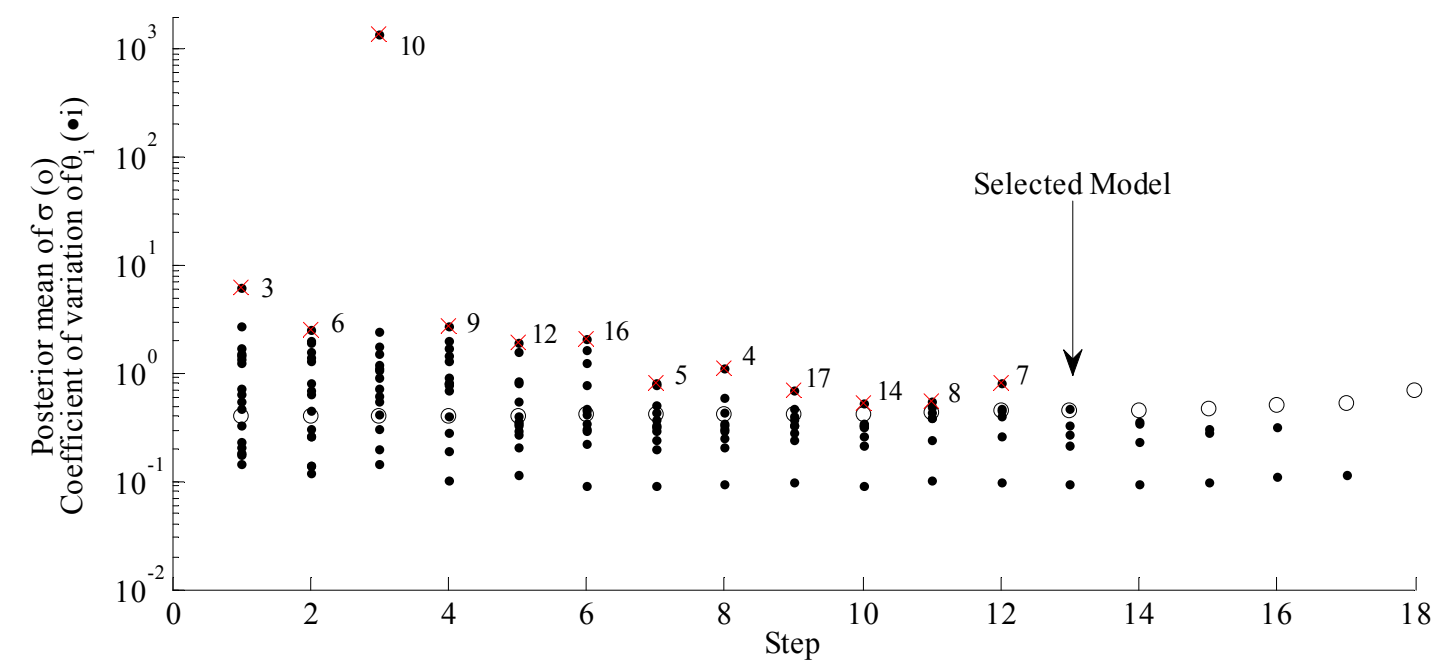

Figure 4-2. Stepwise deletion process for seismic moment demand model, where $(\times)$ indicates term to be removed 
Figure 4-2 shows that after thirteen steps of model reduction the largest COV (for parameter $\theta_{m 15}$ ) is close in magnitude to $\sigma_{m}$ and as it is seen in the figure, further reduction deteriorates the quality of the model. Stopping at this step, the moment demand model is left with six correction terms as shown in Eq. (4-6.)

$$
\begin{aligned}
D_{m}\left(\mathbf{x}, \mathbf{w}, \boldsymbol{\Theta}_{m}\right)= & \hat{d}_{m}(\mathbf{x}, \mathbf{w})+\theta_{m 1}+\theta_{m 2} \hat{d}_{m}(\mathbf{x}, \mathbf{w})+\theta_{m 11} \ln \left(\frac{P G D}{H_{H}}\right)+ \\
& \theta_{m 13} \ln \left(2 \pi \frac{P G D}{P G V \cdot T_{n}}\right)+\theta_{m 15} \ln \left(\frac{C_{s}}{C_{s \max }}\right)+\theta_{m 18} \ln \left(\frac{k_{t}}{k_{f}}\right)+\sigma_{m} \varepsilon_{m}
\end{aligned}
$$

Again, checking all possible subsets of explanatory functions with six terms, the selected model presented in Eq. (4-6) shows the best quality in satisfying the criteria of adjusted $\mathrm{R}^{2}$, Mallows' $\mathrm{C}_{\mathrm{p}}$ and AICc.

\subsubsection{Proposed Probabilistic Seismic Shear and Moment Demand Models}

Once the probabilistic model is selected, the unknown model parameters $\boldsymbol{\Theta}_{k}$ are estimated using a Bayesian approach following Box and Tiao (1992.) The updating rule presented in Eq. (3-7) is used in this section. Similar to the previous section, due to lack of prior information on the unknown parameters, a non-informative prior in the form of $p\left(\boldsymbol{\theta}_{\delta}, \sigma_{\delta}\right) \propto \sigma_{\delta}^{-1}$ (Box and Tiao, 1992) is used in the Bayesian approach. The likelihood function is then constructed using Eq. (3-8), employing the objective information on the model parameters obtained from virtual experiment data. 


\subsubsection{Seismic Shear Demand Model}

With the selected model presented in the Eq. (4-5), the probabilistic shear demand is formulated as the natural logarithm of the shear demand at the base of the tower normalized by the mean value of the yield shear force. As described in previous section, $\hat{V}_{y}$ is defined as

$\hat{V}_{y}=\hat{f}_{y} A_{t}(3 / 4)\left(r_{t o}{ }^{2}+r_{t i}^{2}\right) /\left(r_{t o}{ }^{2}+r_{t o} r_{t i}+r_{t i}{ }^{2}\right)$. Table 4-3 gives the posterior statistics of the model parameters $\boldsymbol{\Theta}_{v}=\left(\boldsymbol{\theta}_{v}, \sigma_{v}\right)$. Figure 4-3 shows a comparison between measured and predicted shear demands based on deterministic (left) and probabilistic (right) models. The solid dots and open triangles represent the equality and lower bound data, respectively. The dashed lines in Figure 4-3(b) delimit the region within one standard deviation of the model. The figure clearly shows an improvement in predicting the demand when using the proposed probabilistic demand model rather than the deterministic model.

\subsubsection{Seismic Moment Demand Model}

Likewise, for probabilistic model presented in the Eq. (4-6), the moment demand is formulated as the natural logarithm of the moment demand at the tower base normalized by $\hat{M}_{y}=\hat{f}_{y} S$, where $S=$ elastic section modulus at tower base. Table $4-4$ gives the posterior statistics of model parameters, and Figure 4-4 shows a comparison between the predicted moment demands versus measured demand based on the deterministic and the probabilistic models. Comments analogous to those made based on Figure 4-3 and are also applicable to the results shown in Figure 4-4. 
Table 4-3. Posterior statistics of the parameters in the shear demand model

\begin{tabular}{lccccccc}
\hline & & Standard & \multicolumn{5}{c}{ Correlation coefficient } \\
\cline { 4 - 8 } Parameter & Mean & Deviation & $\theta_{v 1}$ & $\theta_{v 2}$ & $\theta_{v 9}$ & $\theta_{v 18}$ & $\sigma_{v}$ \\
\hline$\theta_{v 1}$ & -3.12 & 0.428 & 1 & & & & \\
$\theta_{v 2}$ & -0.76 & 0.061 & 0.74 & 1 & & & \\
$\theta_{v 9}$ & 0.30 & 0.040 & -0.11 & -0.39 & 1 & & \\
$\theta_{v 18}$ & -0.24 & 0.041 & 0.71 & 0.12 & -0.09 & 1 & \\
$\sigma_{v}$ & 0.42 & 0.033 & 0.02 & 0.09 & -0.17 & -0.03 & 1 \\
\hline
\end{tabular}

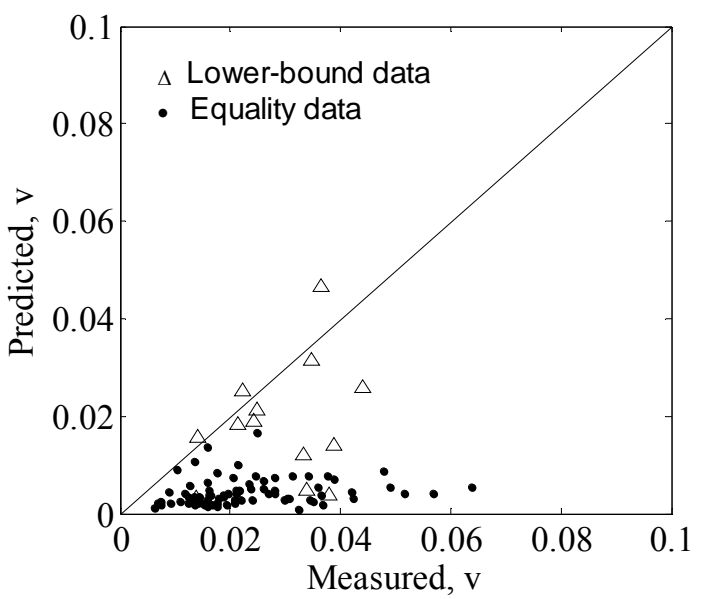

(a)

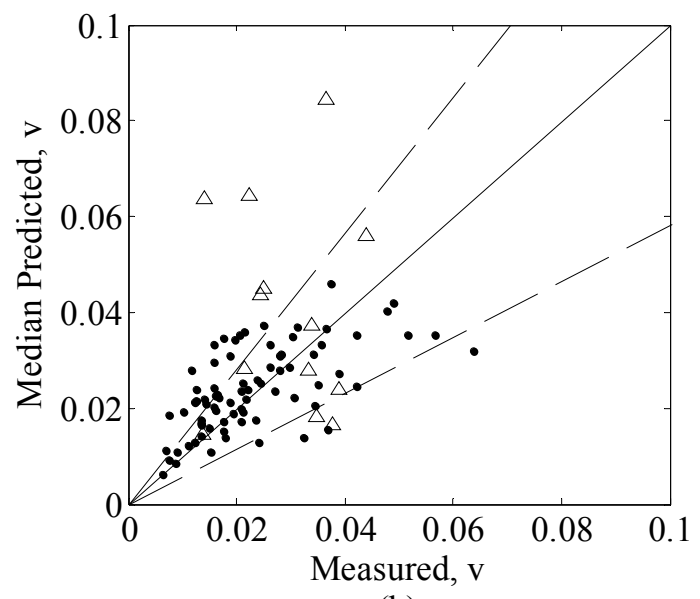

(b)

Figure 4-3. Comparison between measured and predicted seismic shear demands based on (a) deterministic and (b) probabilistic models

Table 4-4. Posterior statistics of the parameters in the moment demand model

\begin{tabular}{lccccccccc}
\hline & & Standard & \multicolumn{7}{c}{ Correlation coefficient } \\
\cline { 5 - 10 } Parameter & Mean & Deviation & $\theta_{m 1}$ & $\theta_{m 2}$ & $\theta_{m 11}$ & $\theta_{m 13}$ & $\theta_{m 15}$ & $\theta_{m 18}$ & $\sigma_{m}$ \\
\hline$\theta_{m 1}$ & -0.32 & 0.640 & 1 & & & & & & \\
$\theta_{m 2}$ & -0.70 & 0.066 & 0.25 & 1 & & & & & \\
$\theta_{m 11}$ & 0.20 & 0.044 & 0.35 & -0.36 & 1 & & & & \\
$\theta_{m 13}$ & -0.24 & 0.080 & -0.49 & 0.46 & -0.62 & 1 & & & \\
$\theta_{m 15}$ & -0.38 & 0.181 & 0.61 & 0.37 & -0.14 & 0.07 & 1 & & \\
$\theta_{m 18}$ & -0.24 & 0.066 & 0.87 & 0.39 & -0.06 & -0.10 & 0.68 & 1 & \\
$\sigma_{m}$ & 0.45 & 0.033 & -0.02 & 0.13 & -0.04 & 0.04 & -0.05 & -0.03 & 1 \\
\hline
\end{tabular}




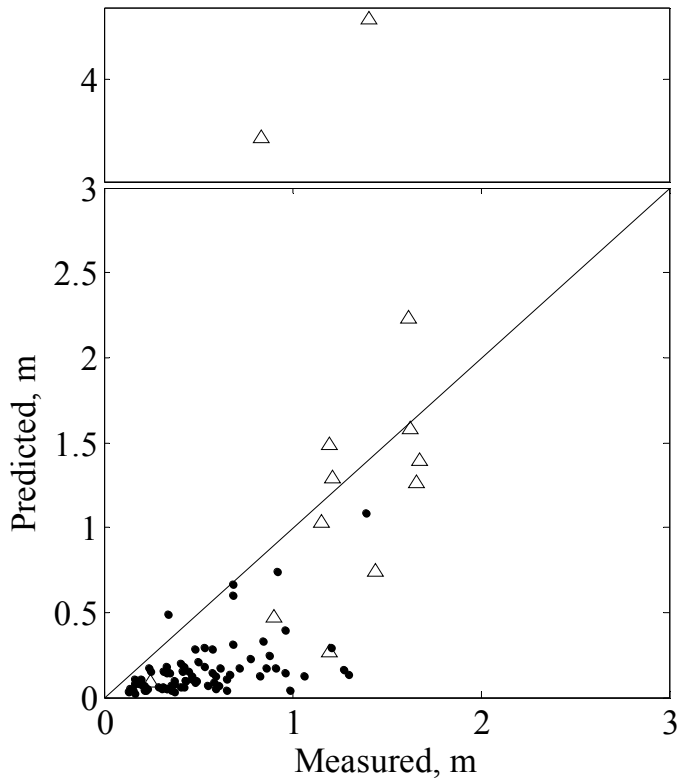

(a)

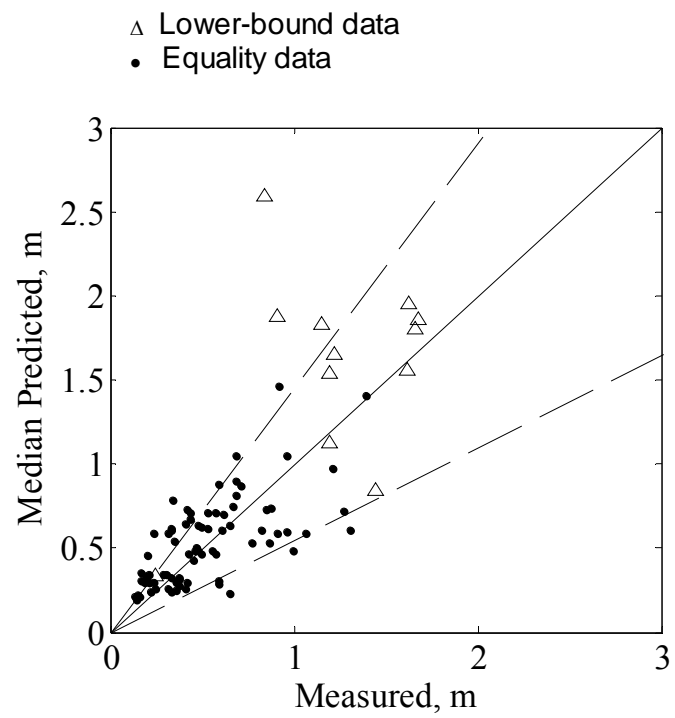

(b)

Figure 4-4. Comparison between measured and predicted seismic moment demands based on (a) deterministic and (b) probabilistic models

\subsection{Seismic Fragility Estimates for an Example Offshore Wind Turbine Support Structure}

Using the developed demand models the fragility of an example offshore wind turbine support structure is assessed. For this purpose, the configuration of a typical 5-MW offshore wind turbine supported by a mono-pile installed in a $20 \mathrm{~m}$ water depth is considered. The structure of interest is called NREL offshore 5-MW baseline wind turbine and its specifications are documented by Jonkman et al. (2009.) Important properties of considered wind turbine are presented in Table 3-7.

This section defines the fragility as the conditional probability of attaining or exceeding a specified performance level for a given vector of $\mathbf{w}$. A predictive estimate of 
the fragility as formulated in Eqs. (3-12) and (3-13) is used, where here $j=y$ or $u$ stands for yield and ultimate performance levels, respectively.

The proposed damage states presented in Table 3-6 are investigated in this section with the exception of the serviceability performance level, given that wind turbines are not expected to produce power while subject to earthquake ground motions. Therefore, the two damage states of (I) and (TO) in the Table 3-6 are combined and a new damage state of (ND) is proposed for not having a significant damage in the support structure. Table 4-5 illustrates the proposed damage states for wind turbines subject to extreme loadings from earthquake. Similar to the previous section, the shear capacity is defined as the shear force in the hollow cross section of the steel tower $C_{v j}=f_{j} A(3 / 4)\left(r_{t o}^{2}+r_{t i}^{2}\right) /\left(r_{t o}^{2}+r_{t o} r_{t i}+r_{t i}^{2}\right)$ where, $f_{j}$ is equal to the steel yield stress, $f_{y}$, for the yield limit, and to the ultimate steel stress $f_{u}$, for the ultimate limit. The yield and ultimate stresses, $f_{y}$ and $f_{u}$ are considered to be lognormal random variables with a mean 300 and $410 \mathrm{MPa}$ (for a structural steel of grade S235 according to EN 10025 (CEN 2004) standard), respectively, and a coefficient of variation of $10 \%$. In addition, $C_{n y}=f_{y} S_{t}$ is used to calculate the yield bending moment capacity. Finally, the ultimate bending moment capacity, $C_{m u}$, is considered to be lognormal random variable with a mean of $390.6 \mathrm{MN}-\mathrm{m}$ and a standard deviation of $39.57 \mathrm{MN}-\mathrm{m}$. The statistics of $C_{m u}$ are obtained using moment-curvature diagrams constructed for the tubular cross section of the tower base, considering the stress-strain curve of structural steel of Grade S235. Monte Carlo simulations are used to estimate the fragility for each failure mode, where all the model parameters and error terms in the developed demand models are considered as 
random variables, in addition to $f_{y}$ and $f_{u}$ as already described.updated damage states and the corresponding performance levels.

Table 4-5. Updated damage states and the corresponding performance levels during earthquake

\begin{tabular}{lll}
\hline Damage state & Description & Performance level \\
\hline No significant damage (ND) & No structural damage. & $\begin{array}{l}\text { Tower base shear or } \\
\text { moment exceeds yield limit }\end{array}$ \\
\hline Permanently out-of-service (PO) & $\begin{array}{l}\text { Support structure yields. } \\
\text { Permanent excessive } \\
\text { deformations. }\end{array}$ & $\begin{array}{l}\text { Tower base shear or } \\
\text { moment exceeds ultimate } \\
\text { limit }\end{array}$ \\
\hline Complete (C) & $\begin{array}{l}\text { Support structure is unable to } \\
\text { carry additional loads }\end{array}$ & \\
\hline
\end{tabular}

Figure 4-5 shows the predictive fragility estimates for the example offshore wind turbine for $H_{s}=1 \mathrm{~m}$ and plotted as a function of the spectral acceleration $S_{a}$ in units of $g$, at the natural period of the support structure $\left(T_{n}=2.5 \mathrm{~s}\right)$ within its linear elastic range, for both the yield and ultimate limit states. Also the damage states are illustrated in the figure. The dotted, solid and dashed lines in the figure show the fragilities for cut-in, rated and cut-out wind speeds, respectively. As shown in the figure, the fragility at the rated wind speed is higher than the fragilities at the other two wind speeds due to the higher wind speed than the cut-in wind speed and higher operational loading than at the cut-out wind speed. However, the contribution of the wind loading is not significant compared to the seismic excitation even for small earthquakes. In addition, the fragility in shear failure mode is found to be 
negligible compared to the bending failure mode, as expected for slender elements like wind turbines towers.

Predictive fragility estimates due to ultimate limit state are also plotted as a function of the wind speed, for different values of spectral accelerations (Figure 4-6) and $H_{s}=1 \mathrm{~m}$. The figure again shows that changes in wind speed do not affect noticeably the probability of failure, especially for large earthquakes. It is also found that the effect of changes in $H_{s}$ on the probability of failure is negligible.

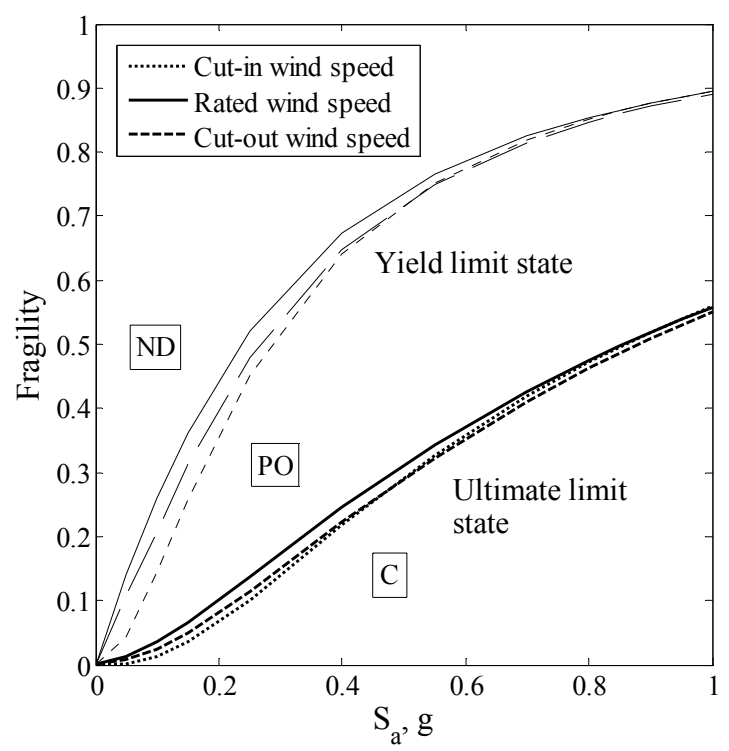

Figure 4-5. Fragility estimates for a typical 5-MW offshore wind turbine as a function of spectral acceleration for both the yield and ultimate limit states 


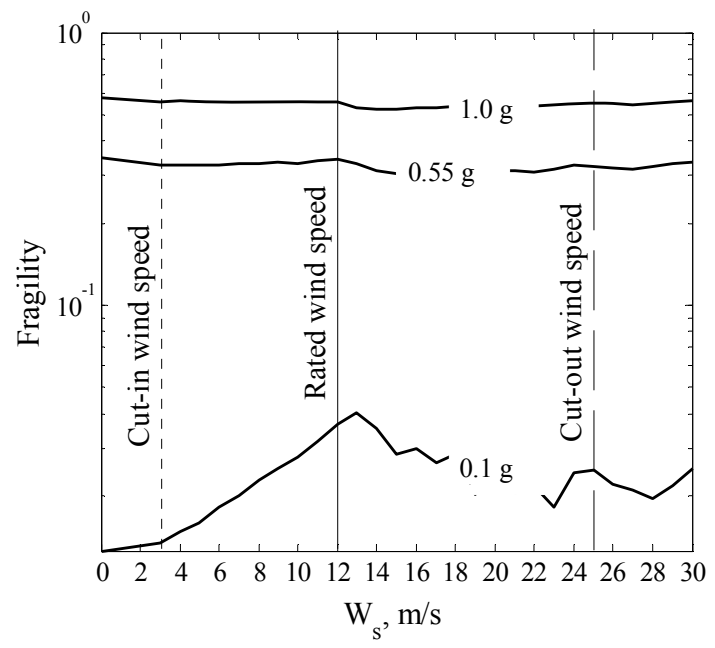

Figure 4-6. Fragility estimates for a typical 5-MW offshore wind turbine as a function of mean wind speed due to ultimate limit state

\subsection{Conclusions}

This section developed probabilistic models for shear and moment demands on the support structure of offshore wind turbines subject to seismic, environmental and operational loads. Employing an experimental design, 100 wind turbine configurations were generated to produce a virtual experiment database used to calibrate the probabilistic models. Detailed finite element analyses were conducted on the generated configurations accounting for the nonlinearities of the soil behavior and soil-structure interaction. A step-wise selection process was used to develop appropriate model forms and a Bayesian approach was used to assess unknown model parameters. The developed probabilistic models account for the relevant aleatory and epistemic uncertainties in predicting the demand quantities of interest.

As an illustration, the developed demand models were used to estimate the fragility of a typical offshore wind turbine support structure. The fragility estimates 
show that bending failure controls the failure of the support structure, while the shear failure mode is negligible. Also the fragility estimates shows that wind speeds within the operational range do not noticeably affect the probability of failure in case of a seismic excitation, especially for large earthquakes. 


\section{MULTI-HAZARD RELIABILITY ASSESSMENT OF OFFSHORE WIND}

\section{TURBINES}

\subsection{Introduction}

Offshore wind turbines installed extensively around the world are subject to different hazards (e.g., earthquake, hurricane, and typhoon) raising concerns about the reliability of the wind turbine support structure subject to multiple hazards. For instance, Japan is the world's $13^{\text {th }}$ largest producer of wind power according to the World Wind Energy Association (Gsänger and Pitteloud 2012), despite having a considerably high occurrence rate of earthquakes and typhoons. Likewise, according to the National Renewable Energy Laboratory (Flowers 2012), California, a highly seismic region, is the third largest wind power producer in the nation. Furthermore, the wind industry is recently considering installing offshore wind farms in the south coast of the United States, and in particular in the Gulf of Mexico, because of the superior wind resources available in this region (Schwartz et al. 2010.) However, a considerably high hurricane occurrence rate in the Gulf of Mexico raises a new concern about the safety of wind turbine support structures subject to hurricane. To investigate the reliability of a wind turbine support structure, all possible hazards that can occur during the wind turbine's life have to be considered. To this end, a probabilistic framework is needed to evaluate the safety of the support structure under multiple hazards and predict its annual probability of failure. The results can assist the wind industry decision makers choosing optimum design and location for future wind energy projects. In addition, the assessment 
of the annual probability of failure of the support structures can be used for an optimal design of wind turbines to maximize the power production and minimize manufacturing, operation and maintenance cost.

This section addresses this need by proposing a probabilistic framework to assess the multi-hazard reliability of offshore wind turbines. As a first step, probabilistic seismic demand models developed in Section 4 are updated using additional virtual experiment data generated for support structures subject to extreme wind loads like those experienced during hurricanes. The virtual experimental data are obtained by developing detailed 3D nonlinear FE models of wind turbines accounting for the dynamic soilstructure interaction. The probabilistic demand models are calibrated using a Bayesian approach. The probabilistic models are then used to develop the fragility curves of wind turbines for given intensity measures of the seismic and wind loading, namely, the spectral acceleration $S_{a}$ and the mean wind speed $W_{s}$. The fragility curves and sitespecific hazard functions are then used to estimate the annual probability of failure. As an illustration, fragility curves and the annual probability of failure are estimated for two identical 5-MW offshore wind turbines one located in the Gulf of Mexico of the Texas' Coast (prone to hurricanes) and one off the California's Coast (a high seismic region.)

The next subsection introduces the probabilistic framework to assess the multihazard reliability of wind turbine support structures. Third subsection discusses the probabilistic formulation for existing demand models. Generation of additional experimental data is explained and then using Bayesian updating rule the existing models are updated. In the fourth subsection, the fragility estimates are presented for an 
example offshore wind turbine support structure for given values of demand parameters, the mean wind speed $W_{s}$ and the spectral acceleration $S_{a}$. This is followed by the analysis of importance and sensitivity measures. Finally, the annual probabilities of failure are estimated for two identical wind turbines located at 1) the Gulf of Mexico of the Texas' Coast (prone to hurricanes) and 2) the California's Coast (a high seismic region.)

\subsection{Multi-hazard Assessment}

According to the total probability rule (Ang and Tang 2007), the probability of failure to meet a specified performance level for a component or system, $P_{f}$, can be written as

$$
P_{f}=\int_{\mathbf{I M}} P(F \mid \mathbf{I M}) f(\mathbf{I M}) d \mathbf{I M}
$$

where $\mathbf{I M}=$ vector of measures of intensity for all possible hazards, $f(\mathbf{I M})=$ the joint probability density function (PDF) of occurrence of $\mathbf{I M}$, and $P(F \mid \mathbf{I M})=$ probability of failure to meet a specified performance level given the occurrence of IM .

The focus of this study is on the two most significant hazards for offshore wind turbines support structures: seismic and wind. Given the intensity measures $\mathbf{I M}=\left(S_{a}, W_{s}\right)$, in which $S_{a}=$ spectral acceleration at the natural period of the wind turbine, and $W_{s}=$ mean wind speed, Eq. (5-1) can be written as

$$
P_{f}=\int_{\mathbf{I M}} P\left(F \mid S_{a}, W_{s}\right) f\left(S_{a}, W_{s}\right) d S_{a} d W_{s}
$$


where $P\left(F \mid S_{a}, W_{s}\right)=$ probability of failure conditioned on $S_{a}$ and $W_{s}$, and $f\left(S_{a}, W_{s}\right)=$ joint PDF of $S_{a}$ and $W_{s}$. Given that the occurrence, or nonoccurrence, of earthquake does not affect the probability of occurrence of any particular level of wind speed and vice versa, $S_{a}$ and $W_{s}$, can be assumed to be statistically independent. Therefore, Eq. (5-2) can be written as

$$
P_{f}=\int_{\mathrm{IM}} P\left(F \mid S_{a}, W_{s}\right) f\left(S_{a}\right) f\left(W_{s}\right) d S_{a} d W_{s}
$$

where $f\left(S_{a}\right)$ and $f\left(W_{s}\right)=$ annual marginal PDF of $S_{a}$ and $W_{s}$, respectively.

\subsubsection{Seismic Contribution to Probability of Failure}

To quantify the probability of future seismic activity at a particular location, the seismic hazard function, $Q\left(S_{a}\right)$, defined as the expected annual frequency of experiencing a spectral acceleration equal to $S_{a}$ or greater, is used. Assuming the arrival of earthquakes at a site is a Poisson process (Frankel at al. 2002), $f\left(S_{a}\right)$, can be expressed in terms of $Q\left(S_{a}\right)$ as

$$
f\left(S_{a}\right)=\exp \left[-Q\left(S_{a}\right)\right]\left[-\frac{d Q\left(S_{a}\right)}{d S_{a}}\right]
$$

The United States Geological Survey (USGS) provides annualized seismic hazard exceedance curves, containing discrete values of $Q\left(S_{a}\right)$ for locations throughout the United States, based on the available information about past earthquakes, 
deformation of the earth crust, geologic site conditions and seismic attenuation relationships (Frankel at al. 2002.)

\subsubsection{Wind Contribution to Probability of Failure}

To develop the annual PDF for wind speed, the PDF for day-to-day wind speed is combined with the one for extreme wind speed during hurricanes. Morgan et al. (2011) investigated annual probability distributions for offshore wind speeds based on statistical analysis of day-to-day 10-min average wind speed data. Wind speed data were recorded at 178 ocean buoy stations around North America, by the National Data Buoy Center (NDBC, 2009.) Based on Morgan et al. (2011), the Bimodal Weibull mixture distribution (BIW) is used to model the day-to-day wind speed. The BIW is a combination of two Weibull (W2) distributions and has two different modes. Using the BIW, the conditional PDF of $W_{s}$ given that there is no hurricane $f\left(W_{s} \mid \bar{H}\right)$ is expressed as

$$
f\left(W_{s} \mid \bar{H}\right)=\varsigma \frac{b_{1} W_{s}^{b_{1}-1}}{a_{1}^{b_{1}}} \exp \left[-\left(\frac{W_{s}}{a_{1}}\right)^{b_{1}}\right]+(1-\varsigma) \frac{b_{2} W_{s}^{b_{2}-1}}{a_{2}^{b_{2}}} \exp \left[-\left(\frac{W_{s}}{a_{2}}\right)^{b_{2}}\right]
$$

where shape $b$ and scale $a$ parameters have subscripts corresponding to the two different modes, $\varsigma=$ mixing parameter, and $\bar{H}$ indicates the event of not occurrence of a hurricane.

Wang (2010) characterized the hurricane event based on the statistical analysis of 4776 hurricanes simulated to occur in 10,000 years with landfall position assumed to 
occur with equal probability along the length of the Texas coastline. Based on Wang (2010), the lognormal distribution is used to model the extreme wind speed associated to hurricanes. The PDF of $W_{s}$ given the occurrence of a hurricane $f\left(W_{s} \mid H\right)$ is written as

$$
f\left(W_{s} \mid H\right)=\frac{1}{\zeta W_{s} \sqrt{2 \pi}} \exp \left[-\frac{1}{2}\left(\frac{\ln W_{s}-\lambda}{\zeta}\right)^{2}\right]
$$

where the location parameter $\lambda$ and scale parameter $\zeta$ are the mean and standard deviation of the natural logarithm of $W_{s}$, respectively. The PDF of $W_{s}$ can now be written as follows, using the total probability rule (Ang and Tang 2007) as

$$
f\left(W_{s}\right)=f\left(W_{s} \mid H\right) P(H)+f\left(W_{s} \mid \bar{H}\right)[1-P(H)]
$$

where $P(H)=$ annual probability of occurrence of a hurricane. With the assumption of arrival of hurricane being a Poisson process, $P(H)=1-\exp \left[-v T_{o}\right]$, in which $v=$ annual occurrence rate of hurricane and $T_{o}=1$ year .

\subsection{Probabilistic Demand Models}

In this section, available seismic demand models (developed in Section 4) are updated using the information obtained from additional virtual experiment data. Additional data are generated using finite element analyses of wind turbine support structures subject to extreme wind speeds in addition to earthquake. 


\subsubsection{Additional Virtual Experiment Data}

Additional virtual experiment data are generated to update the model previously developed in Section 4. A set of representative configurations is selected to generate the virtual experiments using a "space filling" experimental design technique to ensure that the configurations have a good coverage of the design space. See Subsection 3.2.1 for more details on the experimental design and the variables and their considered ranges. The upper limit of the range for the mean wind speed $W_{s}$ is extended to $75 \mathrm{~m} / \mathrm{s}$ to incorporate the extreme wind velocities during hurricane. All other parameters have the same ranges as those considered previously Table 3-1.

Finite element models are developed in ABAQUS to simulate the dynamic response of the support structure of typical offshore wind turbines, subject to different load cases including seismic excitations in addition to day-to-day environmental loads on operating and parked wind turbines, and extreme wind velocities due to hurricanes on parked wind turbines.

Witcher (2005) conducted time domain simulations of wind turbine support structures in different load cases including continuous operation throughout the earthquake, emergency shutdown initiated during the earthquake and parked throughout the earthquake. The results showed a significant difference in the response of operating and parked wind turbines. He concluded that this difference is due to the absence of aerodynamic damping in the parked condition. Comparing the peak loads resulted from time domain analyses with those obtained using frequency domain procedure (based on building design code), Witcher (2005) showed that the results of the two methods of 
time and frequency domain are in reasonably good match during turbine operation. Therefore, he stated that the aerodynamic damping experienced by an operational wind turbine can be close to the typical $5 \%$ value used for the design spectra in building codes. However, the time domain analysis indicated an almost $80 \%$ increase in peak loads over the calculated building code values in the parked case due to the significantly lower aerodynamic damping of a parked wind turbine. Prowell et al. (2008) estimated the structural damping of a $65 \mathrm{MW}$ wind turbine in idling (parked) condition through a full-scale test on the wind turbine mounted on the NEES shake table at the University of California, San Diego. They suggested a value of $0.6 \%$ for the structural damping of a parked wind turbine. In another study on the seismic response of wind turbines, Ishihara and Sarwar (2008) suggested a structural damping of $0.5 \%$ for parked wind turbines.

This study accounts for the aerodynamic damping of an operating wind turbine by considering a $5 \%$ structural damping for the steel tower. The structural damping for parked wind turbines is considered to be $0.5 \%$.

Similar to the FE models developed in previous sections, foundation nonlinearities are considered explicitly in defining nonlinear behavior of the soil and soil-structure interaction. The Mohr-Coulomb plasticity model is used to define the nonlinear behavior of the soil. Soil-pile interaction is modeled using "contact pair", a formulation in ABAQUS to define the nonlinear contact properties of two bodies. Forces at the top of the tower due to the wind only for parked wind turbine and the rotation of the rotor in addition to the wind loads for operating wind turbine is obtained using simulation in FAST. The resulted time history is then used in the finite element model of 
the support structure as an external loading in addition to wave, current and earthquake. Ground motion records are selected from the PEER (Pacific Earthquake Engineering Research Center) NGA database (1999.) The ground motion records are selected based on the bin approach, proposed by Shome and Cornell (1999.) Five bins are used based on the moment magnitude (M) and the closest distance between the record location and the rupture zone $(\mathrm{R})$ to capture all possible characteristics of the earthquake.

\subsubsection{Updated Model}

The most parsimonious forms of the shear and moment demand models were selected in Section 4, based on the posterior statistics of the unknown model parameters, and presented in Eqs. (4-5) and (4-6.) A Bayesian updating approach is then used to update the existing shear and moment demand models following Box and Tiao (1992.) The updating rule presented in Eq. (3-7) is used, where the prior distribution $p\left(\boldsymbol{\Theta}_{k}\right)$ reflects the state of knowledge about $\boldsymbol{\Theta}_{k}$ based on our previous experiments and The posterior distribution $f\left(\boldsymbol{\Theta}_{k}\right)$ incorporates both the previous information about $\boldsymbol{\Theta}_{k}$ included in $p\left(\boldsymbol{\Theta}_{k}\right)$ and the new data included in the likelihood function $L\left(\boldsymbol{\Theta}_{k}\right)$. The likelihood function is constructed using Eq. (3-8) based on the information obtained from additional virtual experiment data. Where similar to previous sections, the virtual experiment data are divided into equality and lower bound data. A threshold of $5 \%$ is considered for drift, such that if the maximum drift during one time history analysis is less than $5 \%$, then the shear and moment data are considered as equality data. If an analysis 
produces a drift that exceeds $5 \%$, then the maximum shear and moment that occurred prior to reaching the $5 \%$ drift are considered as the lower bound data for the shear and moment, respectively.

Table 5-1 gives the updated posterior statistics of the parameters $\boldsymbol{\Theta}_{v}=\left(\theta_{v 1}, \theta_{v 2}, \theta_{v 9}, \theta_{v 18}, \sigma_{v}\right)$ for the shear demand model. The updated statistics include the information content of the new additional data correspond to the extreme wind loads during hurricane. Figure 5-1 shows a comparison between measured and predicted shear demands on the support structure based on the deterministic (left) and probabilistic (right) models. For the probabilistic model the median predictions are shown. Original data used in Section 4 are shown as open circles and triangles for equality and lower bound data, respectively. New additional data generated for wind turbine support structures subject to extreme wind loads due to hurricane are shown using solid dots and triangles for equality and lower bound data, respectively. The dashed lines in the Figure 5-1(b) delimit the region within one standard deviation of the model.

The deterministic model in Figure 5-1(a) is strongly biased on the nonconservative side, because almost all equality data and most of the lower bound data lie below the 1:1 line. However, the proposed probabilistic demand model corrects the bias as shown in Figure 5-1(b.) For a perfect model, all the equality data should be lined up along the 1:1 line and all the lower bound data should lie above the 1:1 line. Using the probabilistic model, the majority of equality data points fall within 1 standard deviation limits and most of lower bound data points are above the 1:1 line. 
Table 5-1. Posterior statistics of the parameters in the shear demand model

\begin{tabular}{lccccccc}
\hline & & Standard & \multicolumn{5}{c}{ Correlation coefficient } \\
\cline { 5 - 8 } Parameter & Mean & Deviation & $\theta_{v 1}$ & $\theta_{v 2}$ & $\theta_{v 9}$ & $\theta_{v 18}$ & $\sigma_{v}$ \\
\hline$\theta_{v 1}$ & -3.05 & 0.480 & 1 & & & & \\
$\theta_{v 2}$ & -0.74 & 0.067 & 0.74 & 1 & & & \\
$\theta_{v 9}$ & 0.26 & 0.044 & -0.15 & -0.41 & 1 & & \\
$\theta_{v 18}$ & -0.23 & 0.046 & 0.74 & 0.15 & -0.12 & 1 & \\
$\sigma_{v}$ & 0.51 & 0.033 & 0.07 & 0.11 & -0.04 & -0.01 & 1 \\
\hline
\end{tabular}

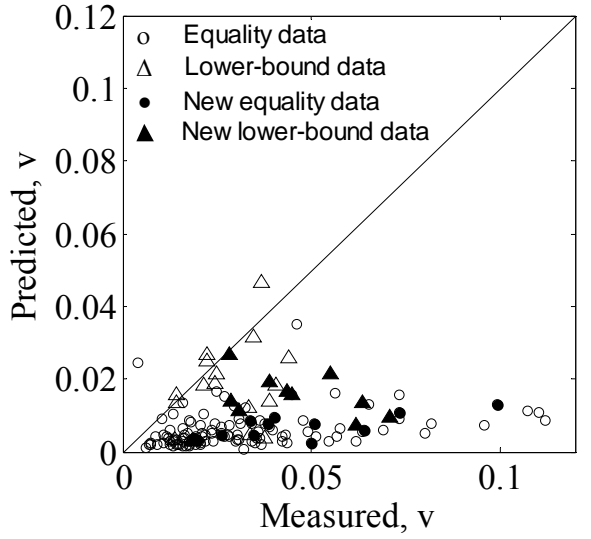

(a)

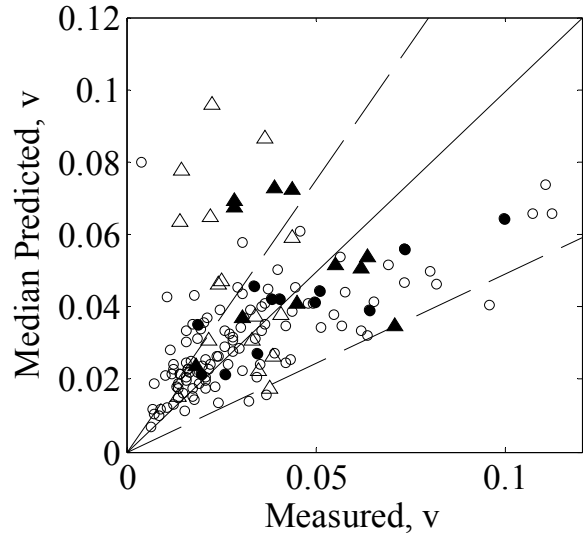

(b)

Figure 5-1. Measured vs. predicted shear demands based on (a) deterministic and (b) probabilistic models

Similarly, Table 5-2 lists the updated posterior statistics of the parameters $\boldsymbol{\Theta}_{m}=\left(\theta_{m 1}, \theta_{m 2}, \theta_{m 11}, \theta_{m 13}, \theta_{m 15}, \theta_{m 18}, \sigma_{m}\right)$ for the moment demand model. Figure 5-2 presents plots of predicted versus measured moment demands based on the deterministic (left) and probabilistic (right) models. The same comments as in Figure 5-1 apply. It is noted that whereas, the deterministic model is strongly biased on the non-conservative side, the proposed probabilistic model corrects the bias. 
Table 5-2. Posterior statistics of the parameters in the moment demand model

\begin{tabular}{lccccccccc}
\hline & & Standard & \multicolumn{7}{c}{ Correlation coefficient } \\
\cline { 5 - 10 } Parameter & Mean & Deviation & $\theta_{m 1}$ & $\theta_{m 2}$ & $\theta_{m 11}$ & $\theta_{m 13}$ & $\theta_{m 15}$ & $\theta_{m 18}$ & $\sigma_{m}$ \\
\hline$\theta_{m 1}$ & -0.57 & 0.694 & 1 & & & & & & \\
$\theta_{m 2}$ & -0.58 & 0.071 & 0.16 & 1 & & & & & \\
$\theta_{m 11}$ & 0.13 & 0.047 & 0.40 & -0.48 & 1 & & & & \\
$\theta_{m 13}$ & -0.13 & 0.088 & -0.54 & 0.49 & -0.68 & 1 & & & \\
$\theta_{m 15}$ & -0.39 & 0.192 & 0.62 & 0.40 & -0.11 & 0.05 & 1 & & \\
$\theta_{m 18}$ & -0.23 & 0.069 & 0.88 & 0.33 & 0.01 & -0.15 & 0.69 & 1 & \\
$\sigma_{m}$ & 0.52 & 0.034 & -0.01 & 0.09 & -0.02 & 0.01 & -0.05 & -0.03 & 1 \\
\hline
\end{tabular}

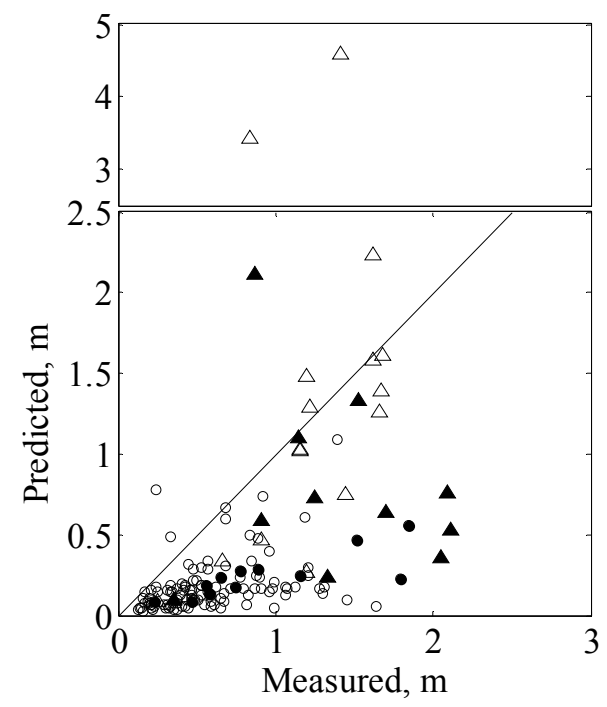

(a)

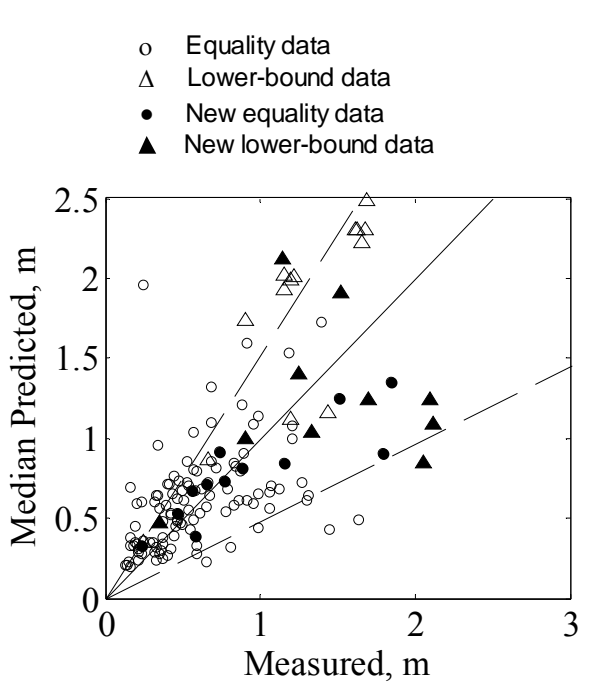

(b)

Figure 5-2. Measured vs. predicted moment demands based on (a) deterministic and (b) probabilistic models

\subsection{Illustration}

As an illustration, the reliability of a typical 5-MW wind turbine with characteristics identical to those for the NREL offshore 5-MW baseline wind turbine, introduced by Jonkman et al. (2009) is explored. The considered wind turbine is installed in a 20 meter 
water depth and assumed to be supported by a mono-pile foundation that is typical for this water depth. Table 3-7 listed a summary of the properties of the configuration of interest.

\subsubsection{Predictive Fragility}

With the updated demand models the predictive fragility is assessed for an example offshore 5-MW wind turbine support structure. The fragility is defined as the conditional probability of exceeding a performance level for a given vector of $\mathbf{w}$. A predictive estimate of the fragility as formulated in Eqs. (3-12) and (3-13) is used, where here $j=y$ or $u$ stands for yield and ultimate performance levels, respectively. The considered damage states and their corresponding performance levels are listed in Table 4-5. Similar shear and bending capacity as those defined in Subsection 4.3 are used in this section for yield and ultimate performance levels. Monte Carlo simulations are used to estimate the predictive fragility for the example wind turbine support structure, where all the model parameters are considered to be normal random variables with statistical properties presented in Tables 5-1 and 5-2. Also, Table 5-3 lists additional random variables in the limit state function and their statistical properties.

Table 5-3. Distribution, mean, and COV for random variables in the limit state function

\begin{tabular}{lccc}
\hline Random variables & Distribution & Mean & COV (\%) \\
\hline$f_{y}$ & Lognormal & 300.0 & 10 \\
$f_{u}$ & Lognormal & 410.0 & 10 \\
$M_{u}$ & Lognormal & 390.6 & 10.13 \\
$C_{s}$ & Lognormal & 109.2 & 30 \\
$k_{t} / k_{f}$ & Lognormal & 0.0020 & 30 \\
\hline
\end{tabular}


The predictive fragility estimates for the wind turbine configuration of interest are presented in Figure 5-3. Figure 5-3(a) presents the predictive fragility estimates plotted as a function of spectral acceleration $S_{a}$, at the natural period of the support structure $\left(T_{n}=2.5 \mathrm{~s}\right)$ within its linear elastic range, for both the yield and ultimate limit states. The significant wave height is set to $H_{s}=1 \mathrm{~m}$. The dotted, solid and dashed lines in the figure show the fragilities for cut-in, rated and cut-out wind speeds, respectively. As shown in the figure, the fragility at the rated wind speed is higher than the fragilities at the other two wind speeds due to the higher wind speed than the cut-in wind speed and higher operational loading than at the cut-out wind speed. However, the contribution of the wind loading in the operational range of wind turbines is not significant compared to the seismic excitation even for small earthquakes. In addition, the fragility in shear failure mode is found to be negligible compared to the bending failure mode, as expected for slender elements like wind turbines towers.

Predictive fragility estimates due to ultimate and yield limit states are also plotted as a function of the mean wind speed, for $H_{s}=1 \mathrm{~m}$ and in absence of earthquake, $S_{a}=0$ (Figure 5-3(b).) The figure shows how the fragility rapidly increases after the cut-out wind speed due to the lack of aerodynamic damping for parked (idle) wind turbine in the presence of high wind speeds. 


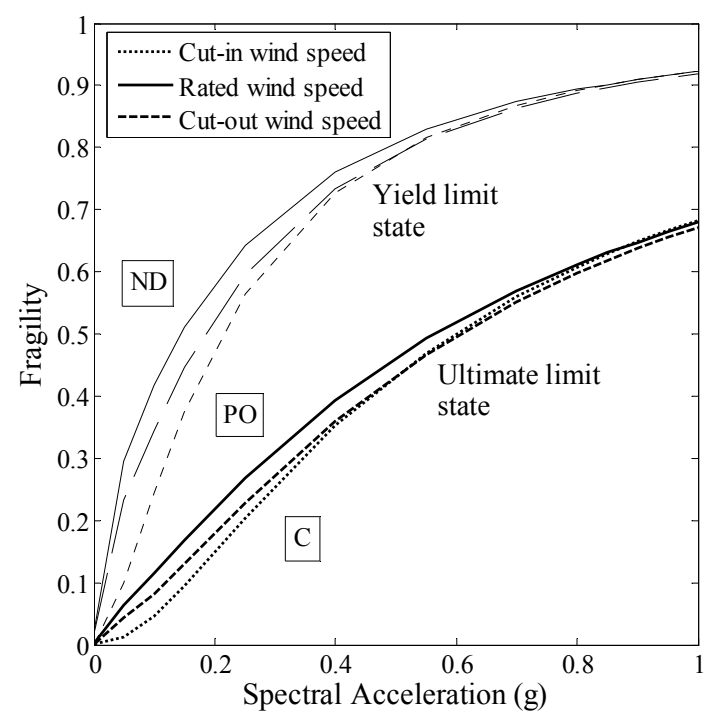

(a)

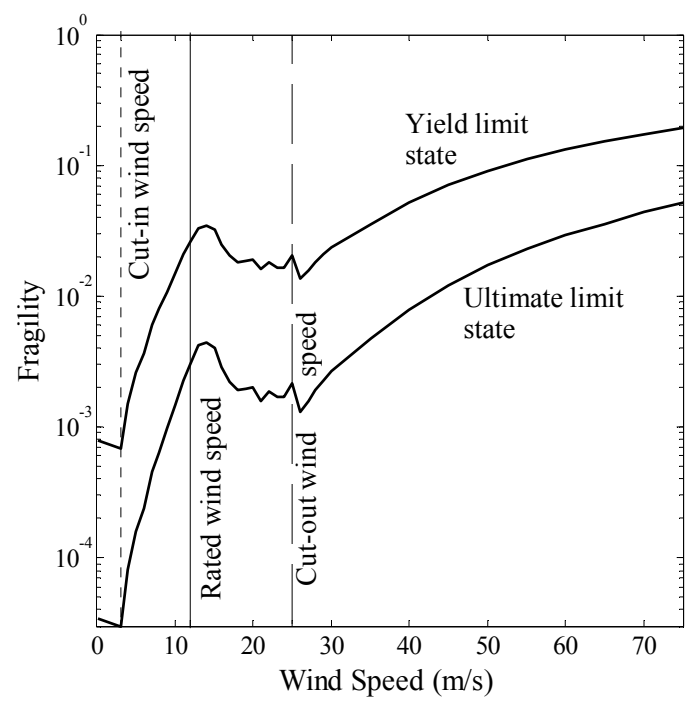

(b)

Figure 5-3. Fragility estimates for a typical 5-MW offshore wind turbine as a function of (a) spectral acceleration and (b) mean wind speed for $S_{a}=0$

\subsubsection{Sensitivity Measures}

A sensitivity analysis is conducted to identify to which parameter(s) the reliability of wind turbine support structure is most sensitive. The sensitivity measures can provide insight into the behavior of support structures and are useful for optimal design and resource allocation. Following Hohenbichler and Rackwitz (1983), the sensitivity of reliability index $\beta$ is defined as the gradient of $\beta$ with respect to a set of parameters $\boldsymbol{\Theta}_{g}$

$$
\nabla_{\mathbf{\Theta}_{g}} \beta=\frac{1}{\|\nabla G\|} \nabla_{\mathbf{\Theta}_{g}} g\left(\mathbf{z}^{*}, \boldsymbol{\Theta}_{g}\right)
$$

where $G(\mathbf{u})=g(\mathbf{z}(\mathbf{u}))=$ limit state function expressed in terms of the standard normal variables. Once $\nabla_{\mathbf{\Theta}_{g}} \beta$ is known, the gradient of the first-order reliability approximation of the failure probability is obtained using chain rule of differentiation as 


$$
\nabla_{\mathbf{\Theta}_{g}} p_{1}=-\varphi(\beta) \nabla_{\mathbf{\Theta}_{g}} \beta
$$

where $\varphi(\cdot)=$ standard normal probability density function.

In this study, $\boldsymbol{\Theta}_{g}=\left[E\left(k_{t} / k_{f}\right), E\left(C_{s}\right), E\left(M_{u}\right), E\left(f_{y}\right), P G D, P G V\right]$. Table 5-4 lists the sensitivity measures for the bending moment failure mode for both ultimate and yield capacity levels. Results show that increasing the tower to foundation stiffness ratio $\left(k_{t} / k_{f}\right)$ is the most effective way of increasing the bending moment reliability (reducing the probability of failure.) Also, the shear wave velocity $C_{s}$ happens to be the second most important parameter, whose increment (increasing the soil stiffness) will increase the reliability of the support structure.

Table 5-4. Sensitivity measures for ultimate and yield bending moment failure modes

\begin{tabular}{lccc} 
& & \multicolumn{2}{c}{$\nabla_{\left(\mathbf{x}_{c}\right)} \beta$} \\
\cline { 3 - 4 } Parameter, $\mathbf{x}_{c}$ & Symbol & Ultimate & Yield \\
\hline Mean of tower to foundation stiffness & $E\left(k_{t} / k_{f}\right)$ & 124.0 & 220.1 \\
Mean of shear wave velocity of soil & $E\left(C_{s}\right)$ & 1.542 & 1.333 \\
Mean of ultimate bending moment & $E\left(M_{u}\right)$ & 0.000 & 0.000 \\
Mean of yield stress of steel & $E\left(f_{y}\right)$ & 0.000 & 0.000 \\
Peak ground displacement & $P G D$ & -0.003 & 0.001 \\
Peak ground velocity & $P G V$ & -0.062 & -0.294 \\
\hline
\end{tabular}

\subsubsection{Importance Measures}

Among several random variables that one may have in a limit state function, some have larger effect on the variance of the limit state function and thus are more important. Der Kiureghian and $\mathrm{Ke}(1985)$ presented a formulation for the measure of importance $\gamma$ as 


$$
\boldsymbol{\gamma}^{T}=\frac{\alpha^{\mathrm{T}} \mathbf{J}_{\mathbf{u}^{*}, \mathbf{z}^{*}} \mathbf{S D}^{\prime}}{\left\|\alpha^{\mathrm{T}} \mathbf{J}_{\mathbf{u}^{*}, \mathbf{z}^{\mathbf{*}}} \mathbf{S D}^{\prime}\right\|}
$$

where $\alpha=$ unit vector at the design point directed towards the failure set, $\mathbf{z}=$ vector of random variables, $\mathbf{J}_{\mathbf{u}^{*}, Z^{*}}=$ Jacobian of the probability transformation from the original space $\mathbf{z}$ to the standard normal space $\mathbf{u}$ with respect to the coordinates of the design point $\mathbf{z}^{*}$, and finally, $\mathbf{S D}^{\prime}=$ standard deviation matrix of equivalent normal variables $\mathbf{z}^{\prime}$ defined by linearized inverse transformation $\mathbf{z}^{\prime}=\mathbf{z}^{*}+\mathbf{J}_{\mathbf{z}^{*}, \mathbf{u}^{*}}\left(\mathbf{u}-\mathbf{u}^{*}\right)$ at the design point. The elements of $\mathbf{S D}^{\prime}$ are the square roots of the corresponding diagonal elements of the covariance matrix $\Sigma^{\prime}=\mathbf{z}^{*}+\mathbf{J}_{\mathbf{z}^{*}, \mathbf{u}^{\mathbf{J}}} \mathbf{J}_{\mathbf{z}^{*}, \mathbf{u}^{*}}^{T}$ of the variables $\mathbf{z}^{\prime}$.

Table 5-5. Importance measures for ultimate and yield bending moment failure modes

\begin{tabular}{lccc} 
& & \multicolumn{2}{c}{$\gamma_{i}$} \\
\cline { 3 - 4 } Random Variable & Symbol & Ultimate & Yield \\
\hline Model parameter for $h_{m 1}$ & $\theta_{m 1}$ & 0.673 & 0.674 \\
Model error $/ \sigma_{m}$ & $\varepsilon_{m}$ & 0.500 & 0.501 \\
Model parameter for $h_{m 18}$ & $\theta_{m 18}$ & -0.421 & -0.419 \\
Model parameter for $h_{m 13}$ & $\theta_{m 13}$ & 0.275 & 0.275 \\
Model parameter for $h_{m 15}$ & $\theta_{m 15}$ & -0.116 & -0.104 \\
Shear wave velocity of soil & $C_{s}$ & -0.110 & -0.115 \\
Ultimate bending moment capacity & $M_{u}$ & -0.098 & 0.000 \\
Model parameter for $h_{m 11}$ & $\theta_{m 11}$ & -0.071 & -0.071 \\
Tower to foundation stiffness ratio & $k_{t} / k_{f}$ & -0.064 & -0.066 \\
Model parameter for $h_{m 2}$ & $\theta_{m 2}$ & 0.014 & 0.014 \\
Standard deviation of moment model error & $\sigma_{m}$ & 0.001 & -0.029 \\
Yield stress of steel & $f_{y}$ & 0.000 & -0.097 \\
\hline
\end{tabular}

Table 5-5 shows the importance measures for the bending failure mode, for both ultimate and yield capacity levels, where $\mathbf{z}=\left(\mathbf{x}_{p}, \boldsymbol{\Theta}_{m}\right)$, in which $\mathbf{x}_{p}=\left(k_{t} / k_{f}, C_{s}, M_{u}\right.$, 
$\left.f_{y}, f_{u}, \varepsilon_{m}\right)=$ set of random variables in the limit state function, in addition to the model parameters $\boldsymbol{\Theta}_{m}$. It is seen that in addition to the model error $\varepsilon_{m}$, some of the model parameters $\theta_{m 1}, \theta_{m 18}$ and $\theta_{m 13}$ are also important random variables that affect the variance of the limit state function. On the other hand, there are random variables in the limit state function that are not important and one can ignore their uncertainty in fragility estimates without significant loss of accuracy. Therefore, vector $\mathbf{z}$ is partitioned in a vector of constant parameters $\mathbf{z}_{c}=\left(k_{t} / k_{f}, C_{s}, M_{u}, f_{y}, f_{u}, \sigma_{m}, \theta_{m 2}, \theta_{m 11}, \theta_{m 15}\right)$, which includes the point estimates of unimportant random variables at their mean values , and a vector of random variables $\mathbf{z}_{p}=\left(\varepsilon_{m}, \theta_{m 1}, \theta_{m 18}, \theta_{m 13}\right)$, so that $\mathbf{z}$ can be written as $\mathbf{z}=\left(\mathbf{z}_{c}, \mathbf{z}_{p}\right)$. Reducing the number of random variables in the limit state function makes the computation of fragilities faster without significant loss of accuracy. Figure 5-4 illustrates the comparison between predictive fragility by Monte Carlo simulations (solid line) and the first-order reliability approximation of fragility with reduced random variables (dotted line.) The figure shows a close match between the two fragility estimates.

\subsubsection{Annual Probability of Failure}

Once the fragilities and the annual probability density functions for seismic and wind hazards are available, the annual probability of failure for a wind turbine support structure can be estimated at any particular locations using the total probability rule (Eq. 


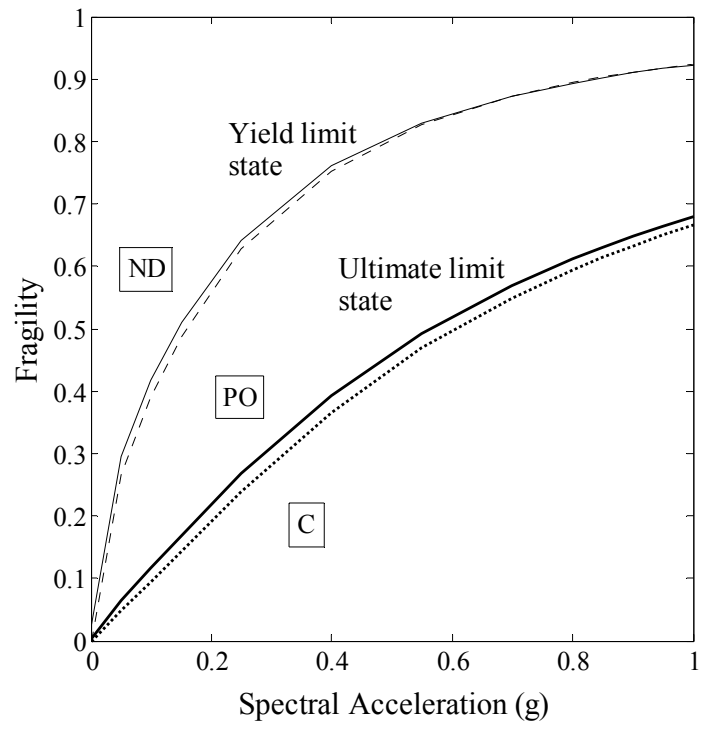

Figure 5-4. Comparison between predictive fragility estimate by Monte Carlo simulations (solid lines) and FORM with reduced number of random variables (dotted lines)

In this study, two locations are selected; Site I in the Gulf of Mexico, about 70 $\mathrm{Km}$ east of Galveston, Texas; with Latitude of $29^{\circ} 25^{\prime} \mathrm{N}$ and Longitude of $94^{\circ} 03^{\prime} \mathrm{W}$ and prone to hurricane, and Site II in the west coast, about $90 \mathrm{Km}$ west of Santa Babara, California; with Latitude of $34^{\circ} 16^{\prime} \mathrm{N}$ and Longitude of $120^{\circ} 42^{\prime} \mathrm{W}$, a high seismic region.

USGS seismic hazard exceedance curves are used for both Sites I and II. Figure 5-5 shows a comparison between the annual PDF of spectral acceleration at the two sites of interest. The figure clearly shows that the annual probability of occurrence of an earthquake in Site II is significantly larger than in Site I.

Day-to-day wind is modeled using the BIW distribution as presented in Eq. (5-5), with the distribution parameters estimated by Morgan et al. (2011) for sample ocean buoy stations at both Sites I and II. Table 5-6 lists the BIW distribution parameters 
considered for the two locations. It is noteworthy that depending on the type of buoy, the wind speed recorded by NDBC is measured at either 5 or 10 meters above sea level (Morgan et al. 2011.) However, the wind speed data at the height of turbine hub are of interest. To obtain the wind speed at the turbine hub height, an empirical approximation of wind speed profile $W_{s}\left(h_{h}\right)$ is used as (DNV/Risø 2002)

$$
W_{s}\left(h_{h}\right)=V_{r}\left(\frac{h_{h}}{H_{r}}\right)^{\eta}
$$

In which, $V_{r}=$ wind velocity at a reference height, where a common choice for the reference height is $H_{r}=10 \mathrm{~m}$. The Guidelines for Design of Wind Turbines (DNV/Risø 2002) suggests a value of $\eta=0.12$ for offshore winds. Then, the probability density functions are update such that the cumulative density values are kept unchanged.

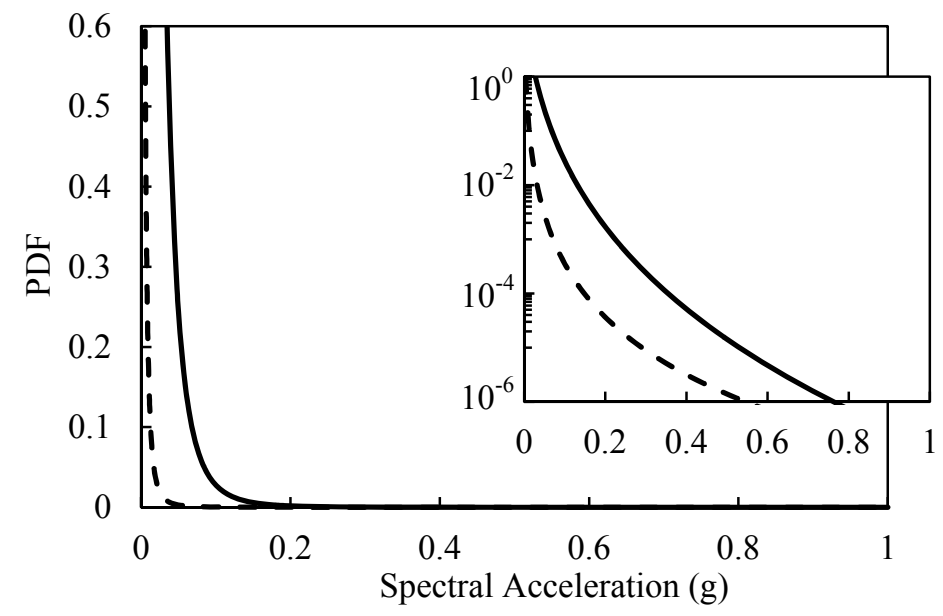

Figure 5-5. Annual probability density function for spectral acceleration at Site I (dashed line) and Site II (solid line) 
Table 5-6. BIW distribution parameters

\begin{tabular}{lcc}
\hline \multirow{2}{*}{ Parameter } & \multicolumn{2}{c}{ Value } \\
\cline { 2 - 3 } & Site I $^{*}$ & Site II $^{\dagger}$ \\
\hline$\beta_{1}$ & 4.455 & 1.694 \\
$\alpha_{1}$ & 5.809 & 5.515 \\
$\beta_{2}$ & 2.067 & 4.405 \\
$\alpha_{2}$ & 6.368 & 9.848 \\
$\omega$ & 0.141 & 0.467 \\
\hline${ }^{*}$ Buoy 42035 [26] & \\
${ }^{\dagger}$ Buoy 46063 [26]
\end{tabular}

As mentioned earlier, Wang (2010) found the lognormal distribution to provide the best fit for the annual PDF of gradient wind speeds during hurricane along the length of the Texas coastline. The gradient level is generally taken as between $500 \mathrm{~m}$ and 2000m. Lee and Rosowsky (2007) summarized the gradient-to-surface wind speed conversion factor for 10-min sustained wind speeds for different locations. They suggested a value of 0.65 for offshore sites. The surface wind speed is the value of wind velocity at 10 meters height above the ground or sea level. However, the wind speed at a wind turbine hub height is of interest in this study. As such, the gradient wind speed is converted to hub height wind speed by first bringing it down to surface level by applying the gradient-to-surface conversion factor and then taking it up to hub height using Eq. (5-11.) Finally for Site I, the lognormal distribution function presented in Eq. (5-6) is used for the hub height wind speed during the hurricane with location and scale parameters of $\lambda=3.348$ and $\zeta=0.34$, respectively.

The occurrence of hurricane is modeled as a Poisson process with an annual occurrence rate of hurricane to be $v=0.1689$ for Site I in the Gulf of Mexico, based on the Historical Hurricane Tracks database at the National Oceanic and Atmospheric 
Administration (NOAA.) NOAA provides a detailed database of hurricanes occurred around the Unites States. For Site II in the West Coast the occurrence rate of hurricane found to be $v=0$. Wind hazard curves are then developed using Eq. (5-7.) Figure 5-6 shows the wind hazard curves for the two particular locations of interest.

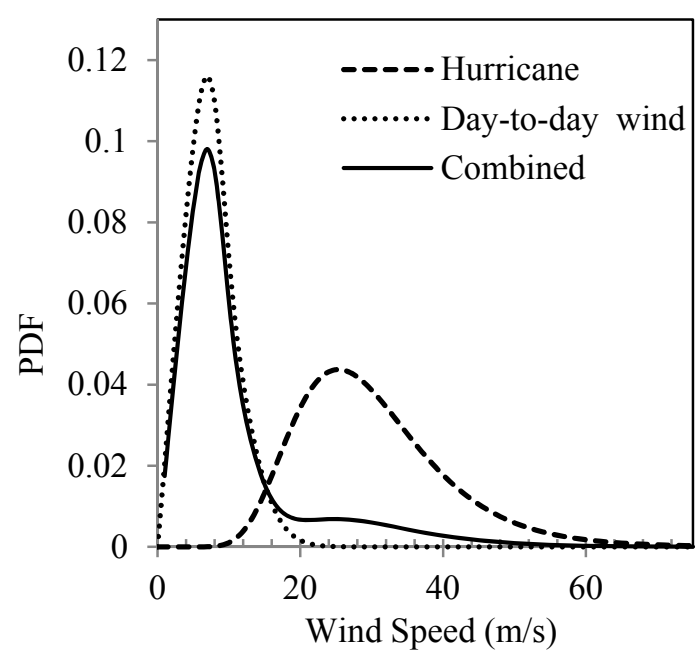

(a)

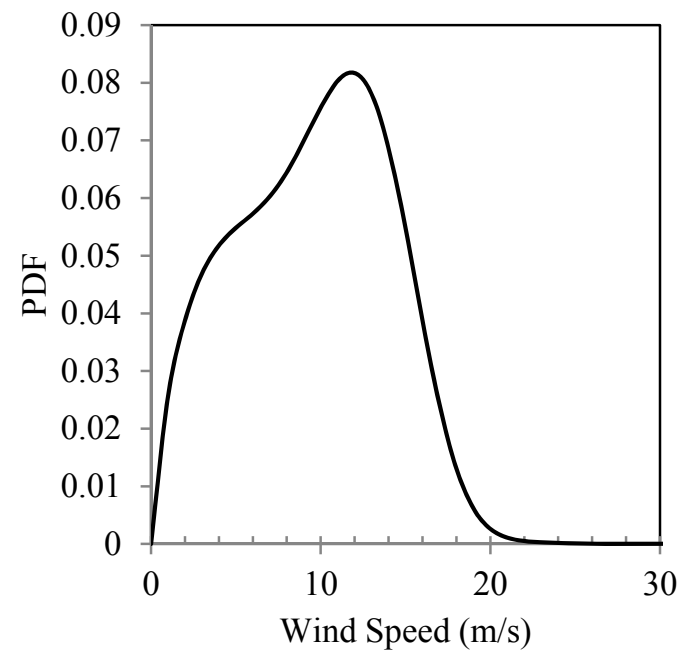

(b)

Figure 5-6. Annual probability density function for wind speeds at (a) Site I and (b) Site II

Table 5-7 lists the annual probabilities of failure for the NREL offshore 5-MW wind turbine subject to different hazards. Table 5-7 shows that even though the occurrence rate of hurricane is much larger at Site I than Site II, the wind hazard alone happens to result in the same failure probabilities for the two sites of interest. Figure 56(b) can explain the reason for the relatively high probability of failure at Site II due to wind hazard. The PDF of day-to-day wind speed for Site II has a considerably higher density around the rated wind velocity (where the wind turbine experiences its maximum 
operational loads) compared to Site I, that has most of its PDF density at the low wind speeds (where the fragility is very small.) Also, with the wind turbines operating at their maximum rate of power production at the rated wind speed, the West Coast happens to be a great location candidate for an offshore wind farm with the superior wind resources for the power production.

Table 5-7. Annual probabilities of failure

\begin{tabular}{|c|c|c|c|c|c|c|c|}
\hline \multirow{3}{*}{$\begin{array}{l}\text { Damage } \\
\text { state }\end{array}$} & \multirow{3}{*}{$\begin{array}{l}\text { Performance } \\
\text { level }\end{array}$} & \multicolumn{6}{|c|}{ Annual probability of failure } \\
\hline & & \multicolumn{2}{|c|}{ Wind Hazard } & \multicolumn{2}{|c|}{ Seismic Hazard } & \multicolumn{2}{|c|}{ Multi-hazard } \\
\hline & & Site I & Site II & Site I & Site II & Site I & Site II \\
\hline \multicolumn{8}{|l|}{ (ND) } \\
\hline & \multirow{2}{*}{$\begin{array}{l}\text { yield limit } \\
\text { exceedance }\end{array}$} & 0.0131 & 0.0139 & 0.0098 & 0.0229 & 0.0143 & 0.0267 \\
\hline \multicolumn{7}{|l|}{$(\mathrm{PO})$} & \\
\hline & \multirow{2}{*}{$\begin{array}{l}\text { ultimate limit } \\
\text { exceedance }\end{array}$} & 0.0016 & 0.0016 & 0.001 & 0.0033 & 0.0017 & 0.0037 \\
\hline (C) & & & & & & & \\
\hline
\end{tabular}

For the case of seismic hazard in presence of day-to-day wind for operating wind turbine, as expected based on the seismic hazard curves, the wind turbine installed in Site II will have a considerably larger probability of failure than the one installed in Site I. Finally with the consideration of multiple hazards (wind and seismic), it is seen that the West Coast with significantly higher seismic risk results in an overall higher failure risk for the wind turbine of interest in this study.

\subsection{Conclusions}

A probabilistic framework is proposed to evaluate the multi-hazard structural reliability of offshore wind turbines. Probabilistic models were developed for shear and moment 
demands on the support structure of wind turbines using the information obtained from detailed three dimensional nonlinear finite element (FE) models of the support structures. The FE models incorporated the aeroelastic interaction as well as the influence of soil-structure interaction in the dynamic response of the support structures. Developed demand models are then used to assess the fragilities of an example offshore wind turbine subject to day-to-day and extreme wind speeds in addition to earthquake. Finally incorporating the hazard information of two particular locations in the Unites States, the probability of failure is evaluated for a typical 5-MW offshore wind turbine subject to day-to-day and extreme wind loads during hurricane in the presence of the seismic risk. The results clearly show a higher failure risk for the wind turbine installed in the West Coast of the United States, due to high seismic risk and high probability density of wind speeds close to rated wind velocity. Although, with the wind turbines operating at their maximum rate of power production at the rated wind speed, the West Coast happens to be a great location candidate for an offshore wind farm with the superior wind resources for the power production. 


\section{PROBABILISTIC ASSESSMENT OF EXCESSIVE DRIFTS FOR OFFSHORE WIND TURBINES}

\subsection{Introduction}

The rapid growth of wind power industry highlights the importance of predicting the cost of energy to evaluate the success of wind energy projects. The cost of energy in a wind energy project is the total cost of the wind farm including the cost of manufacturing and installation, and also operation and maintenance costs. Investigating the reliability of power production of a wind farm by evaluating the performance and serviceability of wind turbines can help ensure the success of an energy project. Adequate reliability can help reduce the need for costly repairs and downtime (Walford 2006.) Excessive vibrational responses can result in adverse effects (such as the unavailability of power production) on the performance and serviceability of wind turbines. For example, excessive wind-induced vibrations at the nacelle of wind turbines can either affect the performance or lead to the malfunction of the acceleration-sensitive components and consequently interfere with the operation of wind turbines (DueñasOsorio and Basu, 2008.) Displacement at the top of the structure can be represented by the drift response. Excessive drifts, similar to other excessive vibrational responses, can cause unfavorable influences on the serviceability of wind turbines (DNV-OS-J101.) The drift threshold for wind turbines will be specified by the manufacturer based on the specific design of each wind turbine. Although a drift ratio of $0.5 \%$ has been considered in some studies as the serviceability threshold for the drifts of wind turbine structures 
(Lavassas et al. 2003), this value might change for different wind turbines. To this end, a framework independent of the value of threshold is needed to evaluate the performance of wind turbines operating under day-to-day loads. The results can assist the wind industry to predict the availability of a wind turbine for power production and accordingly, modify the structural design such that the expected time of staying above a drift threshold is limited to an acceptable level for the unavailability of power production. This section develops models that can predict the probability and expected time that the drift response of a wind turbine stays above certain thresholds for offshore wind turbines supported by mono-pile foundations.

Traditional approaches to estimate the performance of wind turbines rely on historical wind speeds or historical wind turbine failure rates. For example, Walford (2006) and Tavner et al. (2007) investigated the prediction of operation and power production reliability of wind turbines based on historical data of failures and their associated costs. However, a methodology that relies on the structural vibration response of wind turbines will provide a more accurate estimation of their performance.

A number of researchers (Lavassas et al., 2003; Murtagh et al., 2005) have proposed different models to analyze the vibration response of the support structure of wind turbines. Lavassas et al. (2003) studied the dynamic response of wind turbines using the simplified models that lumped the nacelle and rotor as a point mass at the top of the tower. As a result, the aeroelastic interaction is not incorporated in the responses. Modeling the tower and rotating blades as discretized multi-degree-of-freedom entities, Murtagh et al. (2005) incorporated the tower/blade interaction into the equation of 
motion of the tower. Their results showed the exclusion of tower/blade interaction can considerably underestimate the response of the support structure. However with the considered geometry for the blades being much simpler than in reality, the results obtained by Murtagh et al. (2005) primarily indicate behavioral trends rather than realistic responses for the support structure. Furthermore, as mentioned earlier, aeroelastic simulators such as FAST, ADAMS, and GH Bladed were introduced to the wind industry for more accurate analysis of wind turbines incorporating the aeroelastic interaction. However, these simulators are not capable of continuous modeling of the nonlinear foundation behavior and the dynamic soil-structure interaction. To account for the dynamic soil-structure interaction, a continuous modeling of the foundation including the pile and the soil can be conducted using a detailed nonlinear finite element (FE) analysis of the support structure and the foundation. However, both developing and running such FE analyses can be quite expensive and time consuming.

To explore wind turbines unavailability for power production, Dueñas-Osorio and Basu (2008) estimated the annual probability of failure (based on exceeding certain acceleration thresholds) as a measure for unavailability of typical wind turbines. They obtained a distribution for the acceleration response of operating wind turbines for various levels of wind speeds based on the probabilistic description of dynamic properties (damping ratio and natural frequencies) of the wind turbine. However using the mathematical model proposed by Murtagh et al. (2005) towards obtaining the coupled tower/blade response of wind turbines, results obtained by Dueñas-Osorio and Basu (2008) do not represent an accurate estimate for modern wind turbines. Suzuki et 
al. (2011) investigated the progressive drift mode of failure for wind turbine moored systems. Also Ren et al. (2010) evaluated the motion performance of two types of wind turbine floating platforms with combined tension leg and mooring line support systems. Although, those studies are limited to floating foundations and their results cannot be used for other types of wind turbines with fixed support systems.

This section develops a method to predict the probability and expected time that drift response of offshore wind turbines exceeds certain thresholds. To develop the method, an equation based on random vibration theory (Lutes and Sarkani 2003, Vanmarcke 2010) that depends on the mean and standard deviation of the drift response is used. Separate models are developed for the estimation of the mean and standard deviation of drift responses. To incorporate the current knowledge and facilitate the acceptance of the proposed models, the proposed method starts from a simple and yet accurate model that is also commonly used in practice, and modifies it by adding some correction terms. Following an approach consistent with the one used in previous sections, the wind turbine simulator FAST is used as the start point to estimate the response of the support structure. Using a combined modal and multi-body dynamics formulation, even though FAST provides acceptable accuracy in simulating the aerodynamics of the wind turbine and estimating the wind and operational loads, it still fails to provide accurate structural response of the wind turbine support structure. Correction terms are then added to incorporate the missing terms and correct for the bias in FAST simulation. Model parameters used in the correction terms are estimated by comparing the mean and standard deviation of drifts obtained from FAST models and 
those values from corresponding detailed 3D nonlinear FE models in ABAQUS that properly account for the dynamic properties of the support structure and nonlinear behavior of the soil-structure interaction. Therefore, the developed models could provide more unbiased predictions than FAST because the correction terms can compensate for ignoring some important factors such as soil-structure interactions in FAST. In this section, FAST is referred to as a simplified model because of its relative simplicity in structural modeling compared to detailed FE models.

This section has five subsections. After the introduction, the dynamic response of offshore wind turbines is explored. Then, in the third subsection, the model for estimating the probability and expected time of exceeding drift thresholds for offshore wind turbines is developed and its parameters are estimated. The fourth subsection is devoted to the verification of the model and the last subsection presents the summary and conclusions of the section.

\subsection{Dynamic Response of Offshore Wind Turbines}

The focus of this section is on the analysis of the vibration response of wind turbine support structures subject to day-to-day wind, wave, current and the loads from operation of the turbine. Representative configurations of typical horizontal axis offshore wind turbines generated in Section 3 are used in this section. Similar to Section 3, a twostep simulation is conducted to obtain the dynamic vibration response of wind turbines. In the first step, the aerodynamics of the turbine is simulated using FAST. The operational loads on the top of the tower, resultant from FAST, are later used in a 
detailed 3D nonlinear FE analysis conducted in ABAQUS as an external loading in addition to wave and current loads that are modeled separately as described in details in Subsection 3.2.2. The FE models, as developed and described in Section 3, properly account for the nonlinear soil-structure interaction by continuous modeling of the supporting tower, steel pile and the surrounding soil. Dynamic nonlinear analyses in the time domain are conducted on the FE models for calculated day-to-day wind, wave, current and the loads from operation of wind turbines.

\subsection{Development of the Model}

Following Madsen et al. (1999) and Chen et al. (1996), it is assumed that the wind vibration responses of structures are Gaussian stationary processes. Figure 6-1 illustrates the diagnostic plots for the normality assumption for the drift responses of 9 samples of wind turbines used in this study. Also a Kolmogorov-Smirnov (K-S) test (Kolmogorov 1933 ) is conducted as a goodness of fit test to evaluate the validation of the normality assumption. The $\mathrm{K}-\mathrm{S}$ test is conducted under null hypothesis that the sample comes from a normal distribution and results in a p-value that is a measure of the believability of the null hypothesis. The $\mathrm{K}-\mathrm{S}$ test p-values are also presented in Figure 6-1. Plots and p-values presented in Figure 6-1 show that the assumption of normality is a reasonable assumption.

According to Vanmarcke (2010), the probability of exceeding a threshold for a stationary random process is given as

$$
P(X>b)=1-F_{X}(b)
$$


where $X$ is the random process, $b$ is the threshold and $F_{X}(\cdot)$ represents the cumulative distribution of $X$. Also, the probability that $X$ is below $-b$ can be written as

$$
P(X<-b)=F_{X}(-b)
$$
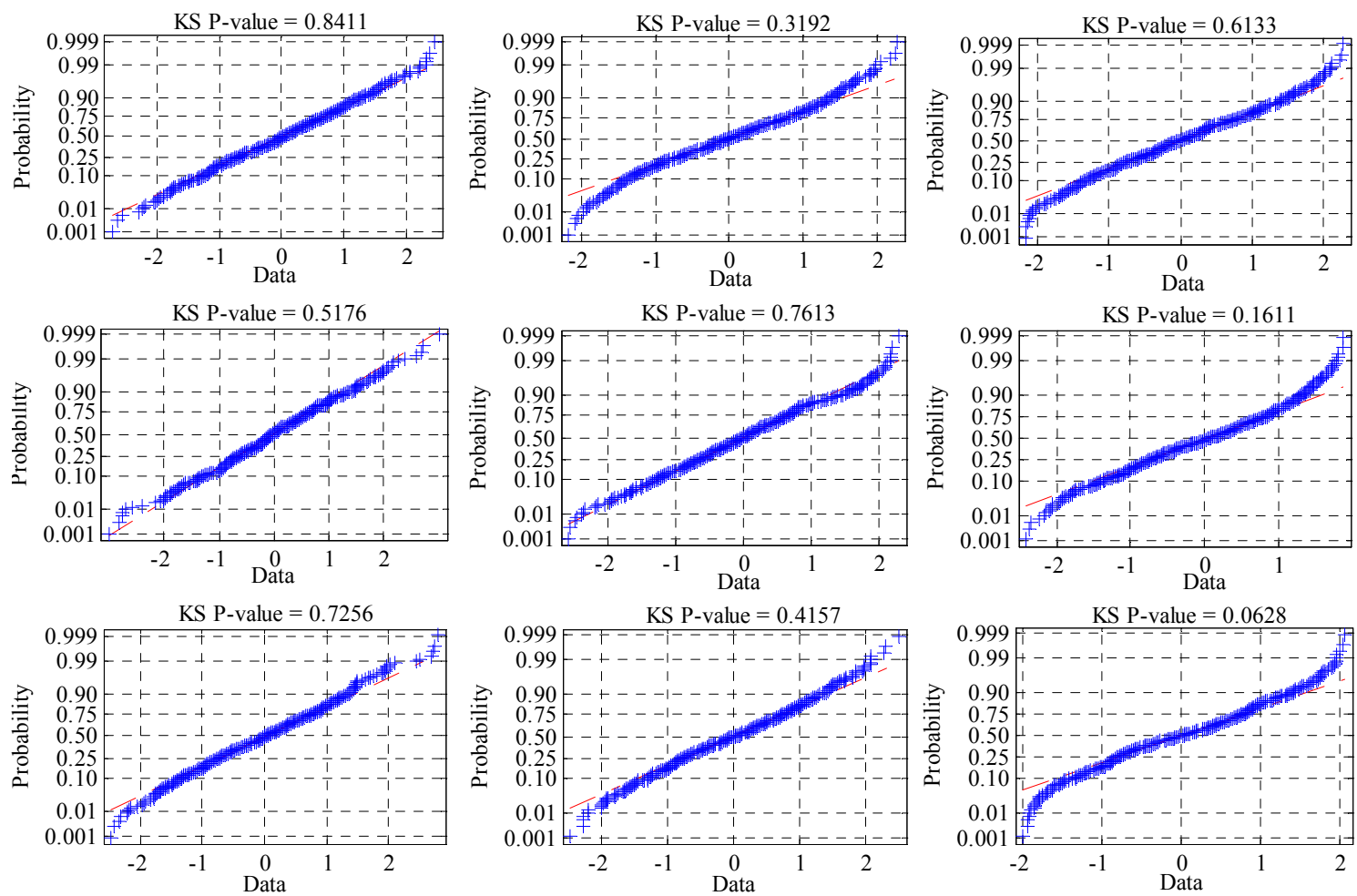

Figure 6-1. Diagnostic plots for normality assumption along with the p-values of Kolmogorov-Smirnov test (KS P-value) for the drift responses of 9 wind turbine configurations considered in experimental design

Since the probabilities in Eq. (6-1) and Eq. (6-2) are mutually exclusive, the following expression can be written

$$
P(|X|>b)=1-F_{X}(b)+F_{X}(-b)
$$


where $|\cdot|$ represents the absolute value operator. Assuming the vibration response of wind turbines as a Gaussian stationary process, Eq. (6-3) can be expressed as follows

$$
P(|X|>b)=1-\Phi_{X}\left(\frac{b-\mu_{X}}{\sigma_{X}}\right)+\Phi_{X}\left(\frac{-b-\mu_{X}}{\sigma_{X}}\right)
$$

where $\Phi_{X}(\cdot)$ is the standard normal distribution and $\mu_{X}$ and $\sigma_{X}$ represent the mean and standard deviation of the responses. Then, the expected value for the time that $X$ exceeds $b$ can be written as

$$
E\left[t_{|X|>b}\right]=\left[1-\Phi_{X}\left(\frac{b-\mu_{X}}{\sigma_{X}}\right)+\Phi_{X}\left(\frac{-b-\mu_{X}}{\sigma_{X}}\right)\right] \cdot T
$$

where $E[\cdot]$ is the expected value operator and $T$ is the total time of the process. Let $v$ denote the normalized estimated time that drift exceeds a given threshold $b$. This is evaluated as the time above the threshold over the total time of the process. Figure $6-2$ compares values of $v$ using Eq. (6-5) with the corresponding values from the time history of the drifts for the models explained in the previous section. Good predictions of Eq. (6-5) presented in Figure 6-2 support the assumption of drift response of wind turbine towers being stationary Gaussian random processes. The point estimates of $\mu_{X}$ and $\sigma_{X}$ obtained from the time history of the drifts from ABAQUS models are used in Eq. (6-5) to obtain the predicted values in Figure 6-2. The accuracy of predictions of Eq. (6-5) depends on using accurate values for $\mu_{X}$ and $\sigma_{X}$.

In practical situations, time history of drifts from detailed 3D nonlinear FE analyses considering soil-structure interaction are not usually available for estimation of 
$\mu_{X}$ and $\sigma_{X}$ of the drift response. However using the drift response time histories resulted from simple fixed base models (e.g., FAST models), the estimation of $\mu_{X}$ and $\sigma_{X}$ might be biased and inaccurate. Therefore, to provide a solution to this problem, models are proposed to estimate $\mu_{X}$ and $\sigma_{X}$ by adding some correction terms that compensate for the errors that arise from using simplified models. For this purpose, the framework proposed by Gardoni et al. (2002) is used to develop unbiased models to estimate $\mu_{X}$ and $\sigma_{X}$ as follows:

$$
\begin{aligned}
& \ln \left(\hat{\mu}_{X}^{u}\right)=\ln \left(\hat{\mu}_{X}\right)+\sum_{i=1}^{n_{\mu}} \theta_{\mu, i} h_{i}(\mathbf{x}) \\
& \ln \left(\hat{\sigma}_{X}^{u}\right)=\ln \left(\hat{\sigma}_{X}\right)+\sum_{j=1}^{n_{\sigma}} \theta_{\sigma, j} h_{j}(\mathbf{x})
\end{aligned}
$$
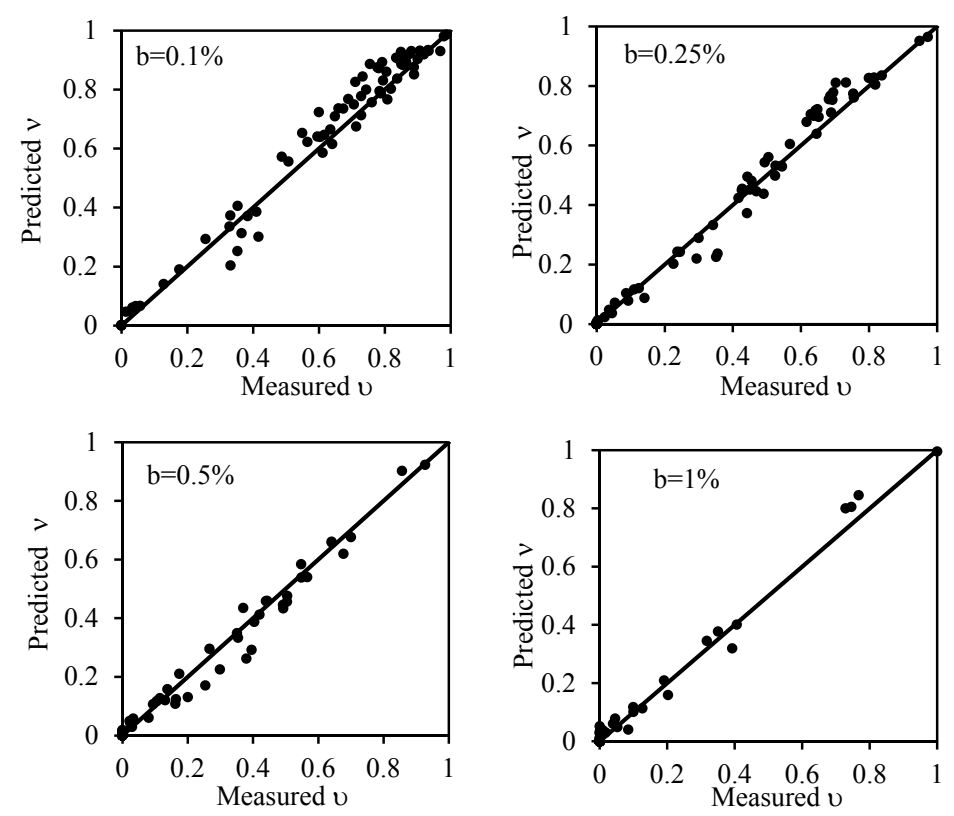

Figure 6-2. Comparison between the predictions of Eq. (6-5) and corresponding measured values for different drift thresholds 
where $\hat{\mu}_{X}^{u}$ and $\hat{\sigma}_{X}^{u}$ are unbiased estimates of $\mu_{X}$ and $\sigma_{X}, \hat{\mu}_{X}$ and $\hat{\sigma}_{X}$ are the estimates of $\mu_{X}$ and $\sigma_{X}$ resulted from simulations in FAST, $\theta_{\mu, i}$ and $\theta_{\sigma, j}$ are parameters of the models, $h_{i}(\mathbf{x})$ and $h_{j}(\mathbf{x})$ are dimensionless explanatory functions, $n_{\mu}$ and $n_{\sigma}$ are the number of explanatory functions and $\mathbf{x}$ is a vector of geometrical, mechanical and physical parameters that are expected to have influence on the responses of the structure. The natural logarithm transformation is used as a variance stabilization technique in Eqs. (6-6) and (6-7) to make sure that the residuals have constant standard deviation.

\subsubsection{Model Selection}

The same candidate explanatory functions as those used in Section 3 are used in this section, except for $h_{k 2}$ that is chosen to be the $\ln \left(\hat{\mu}_{X}\right)$ or $\ln \left(\hat{\sigma}_{X}\right)$ in the developed models for $\mu_{X}$ and $\sigma_{X}$, respectively to capture possible bias in the estimates of corresponding parameters obtained from FAST. Table 6-1 lists the candidate explanatory functions.

To develop parsimonious models, the desire is to keep only the explanatory functions that are strictly needed. Therefore, a model selection process is used to achieve a compromise between the simplicity (few correction terms) and accuracy of the model. The selection of candidate explanatory functions is based on the physics of the problem and engineering judgment. All the parameters that are thought to be ignored or not perfectly considered in the simplified model should be available in the candidate explanatory functions. For example, soil structure interaction is ignored in the 
estimations obtained from FAST. Therefore, the ratio of the stiffness of the tower to the stiffness of foundation, shear wave velocity, cohesion and friction angle of the soil are considered as candidate explanatory functions to capture the bias in the simplified model due to ignoring the soil-structure interaction. If after the model selection, one of these explanatory functions survives in the final model, it implies that soil properties are important terms that are missing in the simplified model.

Table 6-1. Candidate explanatory functions for the developed models for mean and standard deviation

\begin{tabular}{lll}
\hline Explanatory function & Formula & Parameters \\
\hline$h_{k 1}$ & 1 & $k=\mu, \sigma$ \\
$h_{\mu 2}$ & $\ln \left(\hat{\mu}_{X}\right)$ & \\
$h_{\sigma 2}$ & $\ln \left(\hat{\sigma}_{X}\right)$ & \\
$h_{k 3}$ & $\ln \left(W_{s} T_{n} / H_{H}\right)$ & \\
$h_{k 4}$ & $\ln \left(I T_{w}\right)$ & \\
$h_{k 5}$ & $\ln \left(H_{s} / H_{H}\right)$ & \\
$h_{k 6}$ & $\ln \left(T_{p} / T_{n}\right)$ & \\
$h_{k 7}$ & $\ln \left(R D / H_{H}\right)$ & \\
$h_{k 8}$ & $\ln \left(C_{s} / C_{s \max }\right)$ \\
$h_{k 9}$ & $\ln \left(C_{\text {soil }} / E_{\text {soil }}\right)$ & \\
$h_{k 10}$ & $\ln \left[\tan \left(\phi_{\text {soil }}\right)\right]$ & \\
$h_{k 11}$ & $\ln \left(k_{t} / k_{f}\right)$ & \\
\hline
\end{tabular}

A model selection process is used to construct accurate and parsimonious models for the mean and standard deviation of drifts. In the absence of lower bound data, statistical model selection criteria including Root Mean Squared Error (RMSE), the adjusted $R^{2}$, Mallows' $C_{p}$, the Corrected Akaike's Information Criterion (AICc) and Bayesian Information Criterion (BIC) (Schwarz 1978) are used as the selection criteria. RMSE is a commonly used measure of accuracy of the model, the adjusted $R^{2}$ and AICc are two selection criteria that capture how closely the model fits the data, BIC is closely 
related to AICc with a larger penalty term and $C_{p}$ is used to check potential overfitting of the data. More details about the model selection criteria used in this study can be found in statistical textbooks such as Burnham and Anderson (2002.) Among all possible models that have the same number of explanatory functions, the one with highest adjusted $R^{2}$ or lowest $C_{p}$, AICc, BIC or RMSE provides the most accurate predictions. The advantage of this model selection method over step-wise deletion process is the possibility to select the "best" model with $n_{k}$ explanatory functions from a set of candidate models using all possible subsets of explanatory functions that have $n_{k}$ members. Selection of the number of the explanatory functions in the final forms of the models is then a trade-off between simplicity and accuracy of the model because choosing a larger number of explanatory functions might result in a more accurate but more complex model. To choose the optimum number of the explanatory functions, the value of all the selection criteria for the best models with $n_{k}$ explanatory functions is recorded with $n_{k}$ as shown in Figures 6-3 and 6-4. Based on the presented graphs, 5 and 4 explanatory functions are considered for the mean and standard deviation models, respectively. Then the parameters of the models can be estimated using a regression analysis. In the regression analysis, the error term is the dependent variable and those explanatory functions that have survived in model selection process, are considered as predictors. The error term is defined as the difference between mean or standard deviation obtained from detailed 3D nonlinear models and the corresponding values obtained from FAST models. 
As a result, the final models for the mean and standard deviation of drifts can be expressed as follows:

$$
\begin{aligned}
& \hat{\mu}_{X}^{u}=\exp \left[\left(1+\theta_{\mu, 2}\right) \ln \left(\hat{\mu}_{X}\right)+\theta_{\mu, 3} \ln \left(W_{s} T_{n} / H_{H}\right)+\theta_{\mu, 5} \ln \left(H_{s} / H_{H}\right)+\theta_{\mu, 6} \ln \left(T_{p} / T_{n}\right)+\theta_{\mu, 8} \ln \left(C_{s} / C_{s \max }\right)\right] \\
& \hat{\sigma}_{X}^{u}=\exp \left[\left(1+\theta_{\sigma, 2}\right) \ln \left(\hat{\sigma}_{X}\right)+\theta_{\sigma, 3} \ln \left(W_{s} T_{n} / H_{H}\right)+\theta_{\sigma, 5} \ln \left(H_{s} / H_{H}\right)+\theta_{\sigma, 6} \ln \left(T_{p} / T_{n}\right)\right]
\end{aligned}
$$

Table 6-2 presents the statistical properties of the model parameters in Eqs. (6-8) and (6-9.) In the presence of additional field or virtual experiment data in the future, the estimated properties of the model parameters presented in Table 6-2 can be updated using a Bayesian updating rule (Ang and Tang, 2007.)
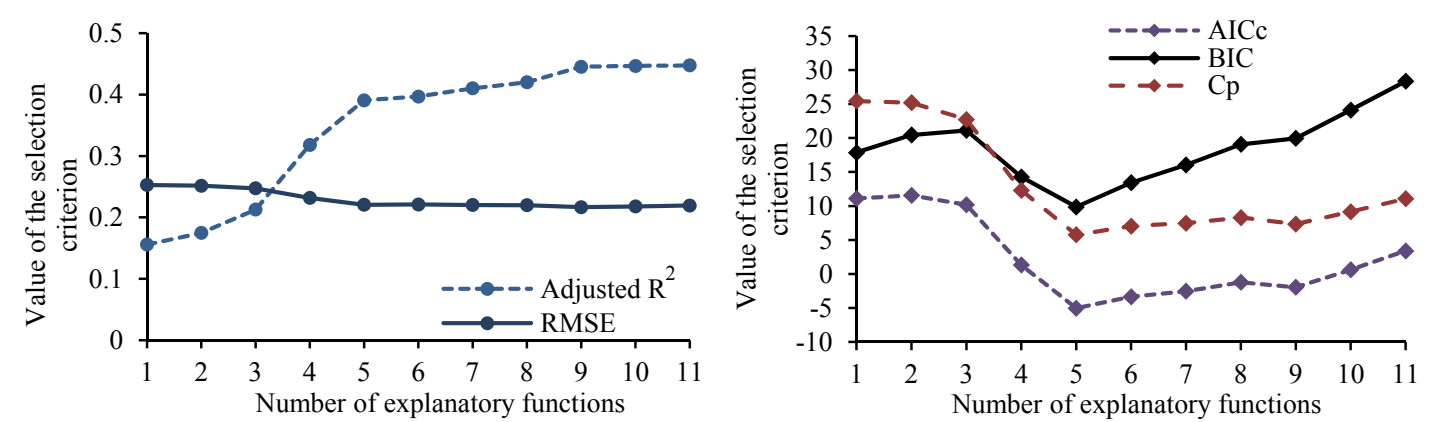

Figure 6-3. Values of the selection criteria versus number of explanatory functions for the mean model
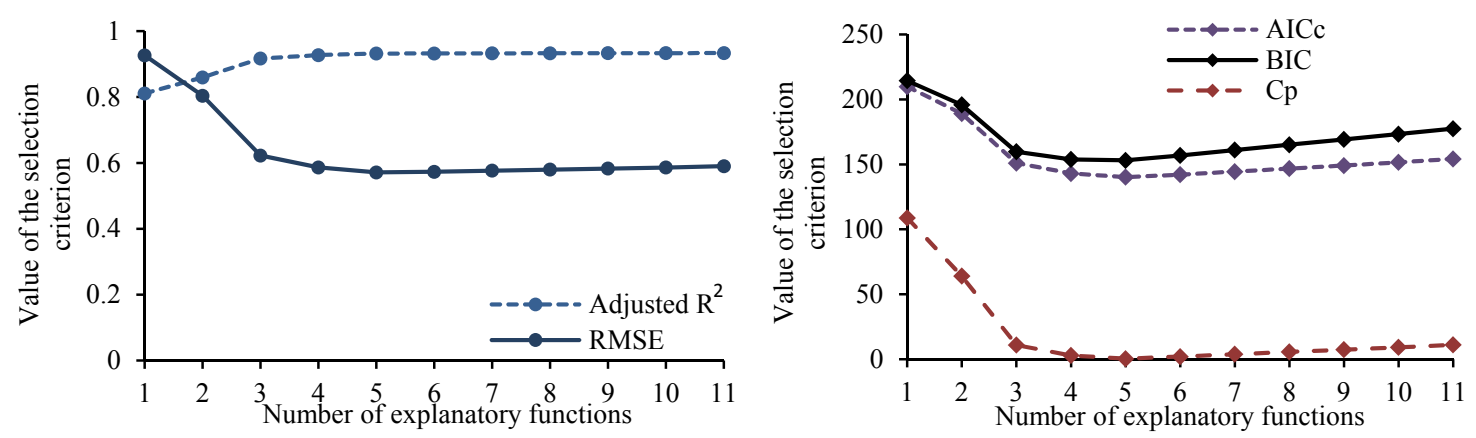

Figure 6-4. Values of the selection criteria versus number of explanatory functions for the standard deviation model 
Table 6-2. Statistical properties of model parameters

\begin{tabular}{ccc}
\hline Parameter & Mean & Standard deviation \\
\hline$\theta_{\mu, 2}$ & -0.215 & 0.052 \\
$\theta_{\mu, 3}$ & 0.096 & 0.043 \\
$\theta_{\mu, 5}$ & 0.364 & 0.069 \\
$\theta_{\mu, 6}$ & -0.535 & 0.114 \\
$\theta_{\mu, 8}$ & -0.259 & 0.088 \\
$\theta_{\sigma, 2}$ & -0.688 & 0.047 \\
$\theta_{\sigma, 3}$ & 0.391 & 0.123 \\
$\theta_{\sigma, 5}$ & 0.787 & 0.085 \\
$\theta_{\sigma, 6}$ & -1.282 & 0.155 \\
\hline
\end{tabular}
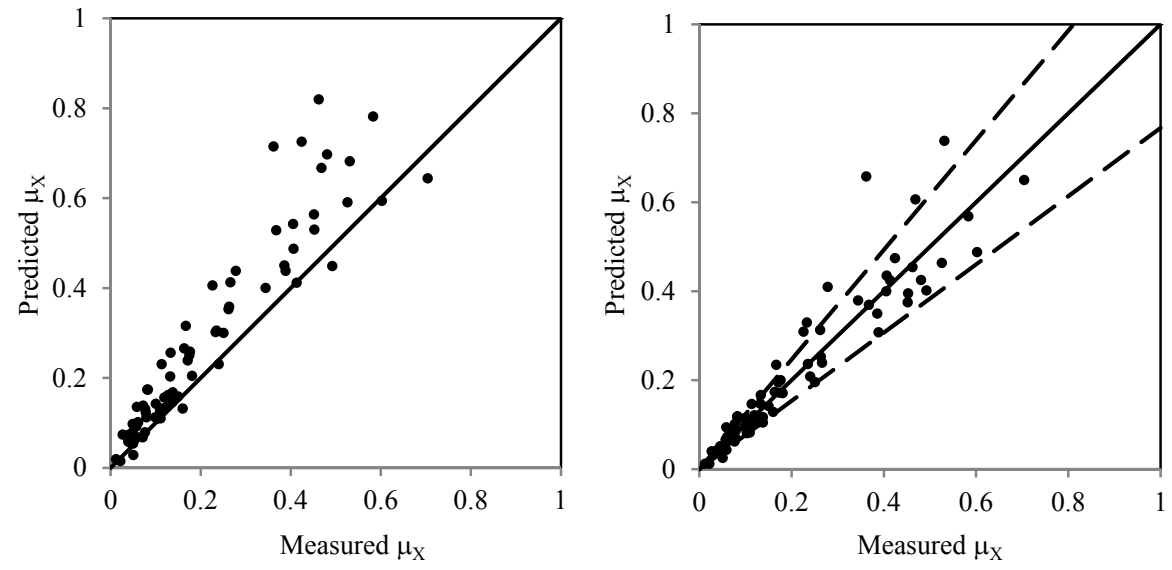

Fig 6-5. Measured versus predicted mean of the drifts based on FAST models (left) and proposed models (right)
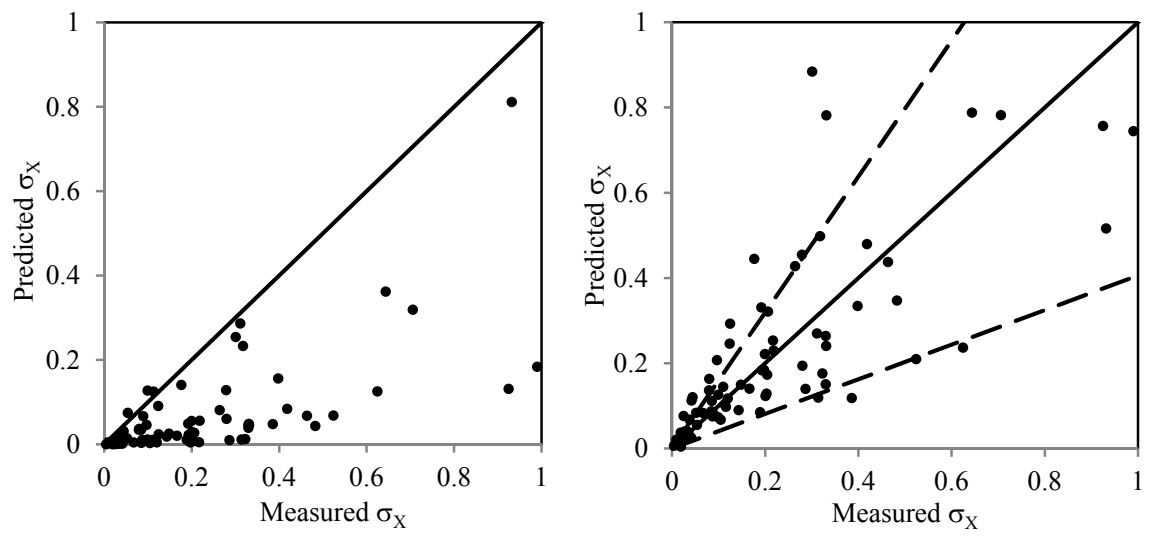

Fig 6-6. Measured versus predicted standard deviation of the drifts based on FAST models (left) and proposed models (right) 


\subsubsection{Model Accuracy}

Figures 6-5 and 6-6 show predicted versus measured values for the mean and standard deviation of the drifts, respectively. The predicted values in the left graphs of Figures 65 and 6-6 are obtained from simplified models in FAST and the right graphs are plotted using the predictions of the proposed models presented in Eqs. (6-8) and (6-9) with the point estimate of the parameters at their mean values. The dashed lines in Figures 6-5(b) and 6-6(b) delimit the region within one standard deviation of errors for developed models. Figures 6-5 and 6-6 reveal that the FAST estimates of the mean and standard deviations of the drift response are over- and under-estimated, respectively while the proposed models properly correct the bias in the estimates obtained from FAST. Using Eq. (6-5) and with the FAST estimations of mean and standard deviations, Figure 6-7 presents the normalized estimated time that drift response exceeds given drift ratio thresholds in a range of $[0.1 \%, 1 \%]$ versus the corresponding measured values from accurate $\mathrm{FE}$ analyses. The range of $b$ values used is quite broad and includes the value of $0.5 \%$ for the drift ratio threshold that has been considered in some studies (Lavassas et al. 2003) as the serviceability threshold for drift of wind turbine support structures. It is obvious from this figure that the estimates of response obtained from FAST simulations do not provide a good prediction for the amount of time that drifts exceed a certain threshold. Figure 6-8 presents similar graphs with predicted means and standard deviations from proposed models in Eqs. (6-8) and (6-9.) Figure 6-8 shows that even the predictions for the estimated time that drifts exceeds a threshold are not perfect using the developed models in this section, but they are considerably less biased compared to the 
results obtained using simplified models. To compare the errors in the predictions shown in Figures 6-7 and 6-8, RMSE that is a measure of the accuracy of predictions is calculated for the predictions presented in those figures. Table 6-3 lists the values of RMSE for the two cases, which confirms that using proposed models produces less error than using simplified model in FAST.

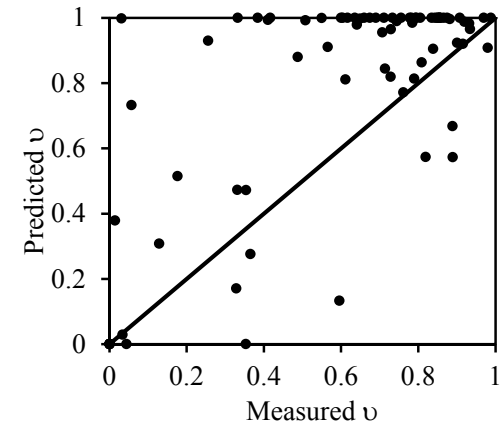

(a)

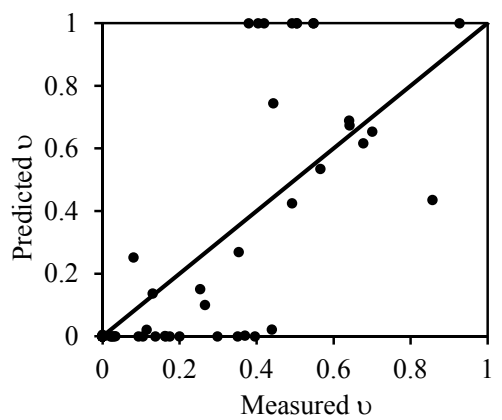

(c)

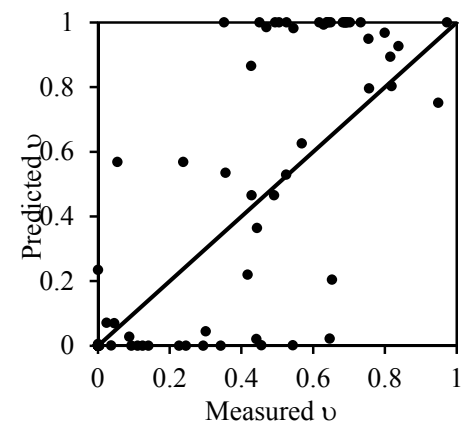

(b)

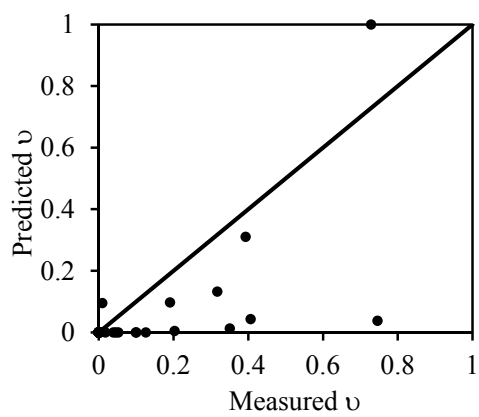

(d)

Figure 6-7. Predicted versus measured values of $v$ based on FAST models for drift ratio thresholds of (a) $b=0.1 \%$, (b) $b=0.25 \%$, (c) $b=0.5 \%$ and (d) $b=1 \%$

Table 6-3. RMSE values for the predictions of $u$

\begin{tabular}{lcccc}
\hline & \multicolumn{4}{c}{ Drift ratio threshold } \\
\cline { 2 - 5 } Model & $0.1 \%$ & $0.25 \%$ & $0.5 \%$ & $1 \%$ \\
\hline Simplified model in FAST & 0.3 & 0.28 & 0.21 & 0.11 \\
Proposed model & 0.12 & 0.14 & 0.12 & 0.09 \\
\hline
\end{tabular}




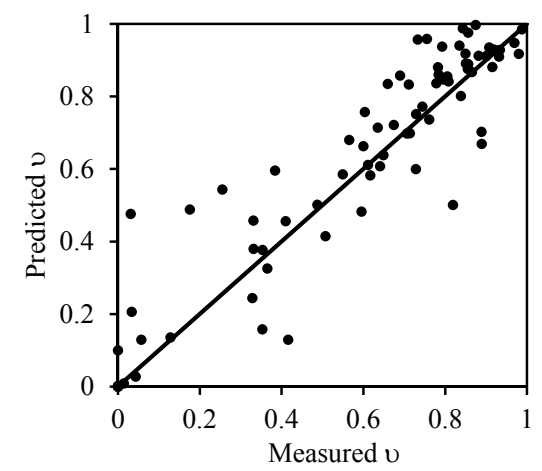

(a)

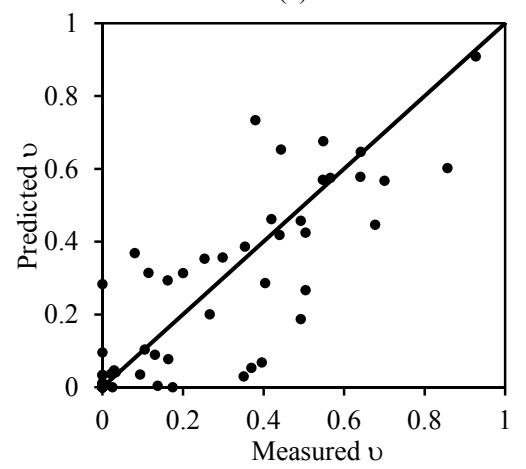

(c)

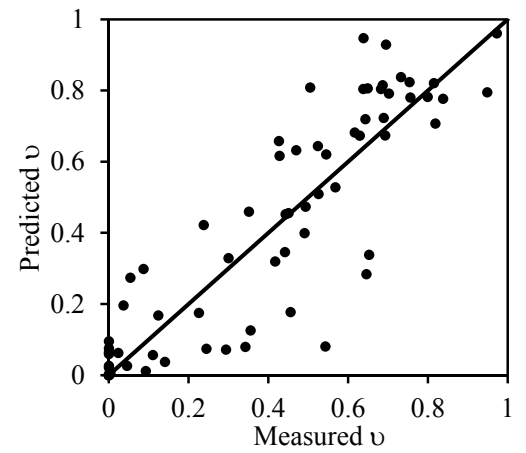

(b)

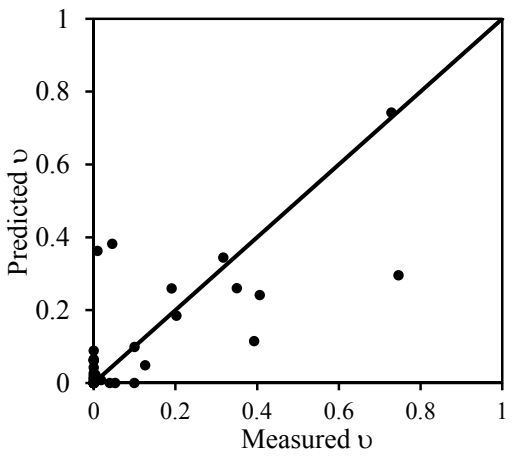

(d)

Fig 6-8. Predicted versus measured values of $v$ based on proposed models for drift ratio thresholds of (a) $b=0.1 \%$, (b) $b=0.25 \%$, (c) $b=0.5 \%$ and (d) $b=1 \%$

\subsection{Verification}

To verify the developed models in this section, a new typical wind turbine configuration that was not included in the configurations used for model development is considered. For the selected wind turbine, detailed 3D nonlinear FE model in ABAQUS and the corresponding model in FAST are created. Table 6-4 shows the properties of the considered example wind turbine.

Fig. 6-9 shows the schematic 3D curve for the probability of exceeding different thresholds in different wind speeds for the considered wind turbine. Fig. 6-10 represents cuts of Fig. 6-9 with the calculated probability of exceeding thresholds for two specific 
levels of wind speed, namely, the rated and cut-out wind speeds. The probability of exceeding a specific threshold, as shown in Fig. 6-10, increases with the wind speed up to the rated wind speed and after that, due to the activation of pitch control system, decreases suddenly and then increases again gradually with the increase of wind speed.

Table 6-4. Properties of the example wind turbine considered for the verification of the developed models

\begin{tabular}{ll}
\hline Property & Value \\
\hline Rotor diameter & $88.29 \mathrm{~m}$ \\
Hub height & $73.18 \mathrm{~m}$ \\
Water depth & $24.00 \mathrm{~m}$ \\
Pile penetration & $24.77 \mathrm{~m}$ \\
Cut-in, rated, cut-out wind speed & $3 \mathrm{~m} / \mathrm{s}, 11.4 \mathrm{~m} / \mathrm{s}, 25 \mathrm{~m} / \mathrm{s}$ \\
Tower steel type & $\mathrm{S} 355$ \\
Tower top diameter and wall thickness & $2.00 \mathrm{~m}, 0.017 \mathrm{~m}$ \\
Tower base diameter and wall thickness & $5.60 \mathrm{~m}, 0.047 \mathrm{~m}$ \\
\hline
\end{tabular}

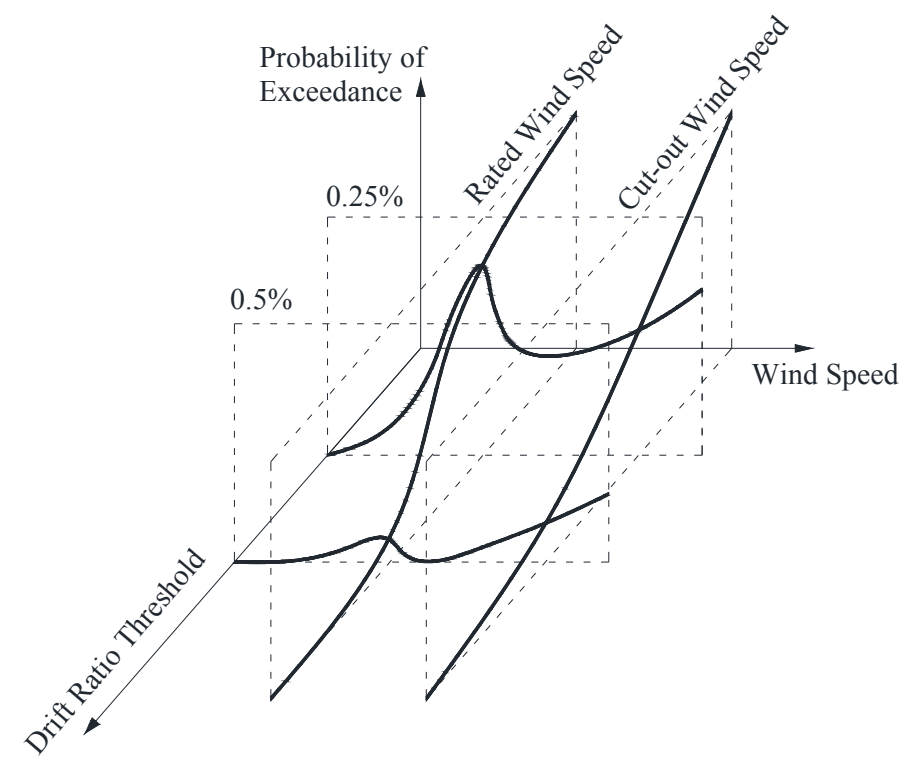

Figure 6-9. Schematic 3D curve for the probability of exceeding drift ratio thresholds versus threshold values and wind speed for the example wind turbine 
Figure 6-11 represents the cuts of Figure 6-9, perpendicular to those in Figure 610, with the calculated probability of exceeding drift ratio thresholds for two specific levels of threshold $(0.25 \%$ and $0.5 \%$.) Figures $6-10$ and $6-11$ reveal that the proposed models generally provide better predictions for the studied example wind turbine.

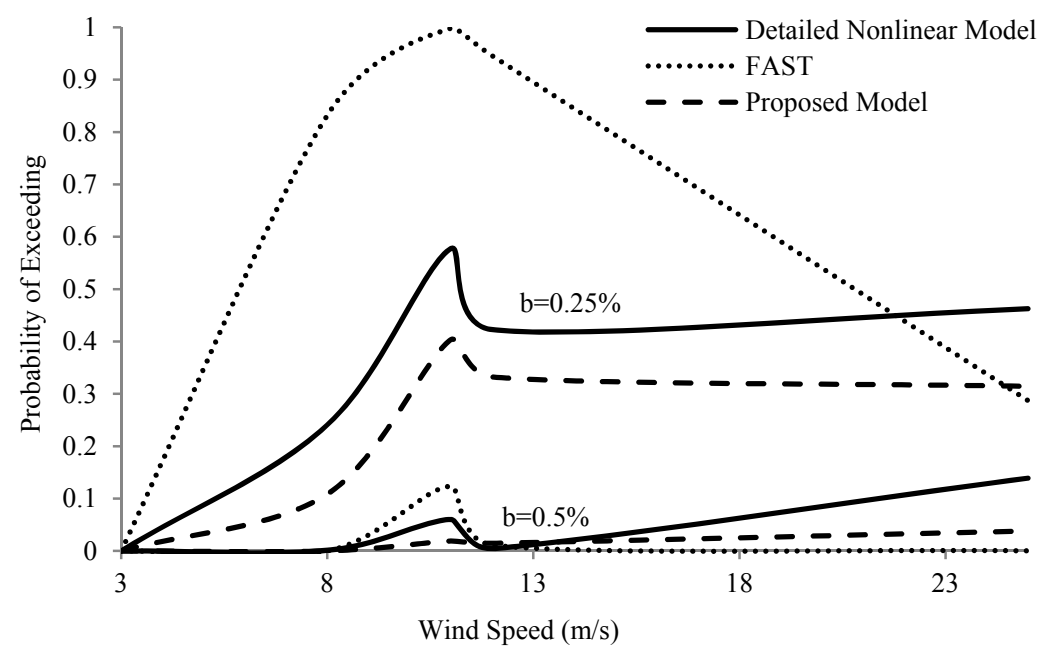

Figure 6-10. Probability of exceeding drift ratio thresholds versus wind speed for the example wind turbine

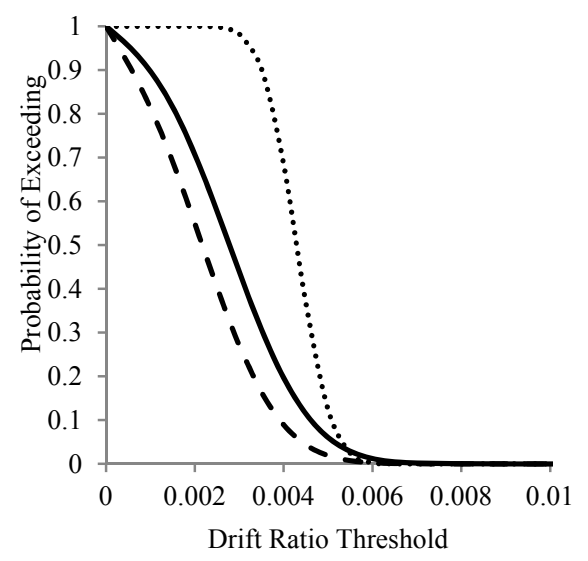

(a)

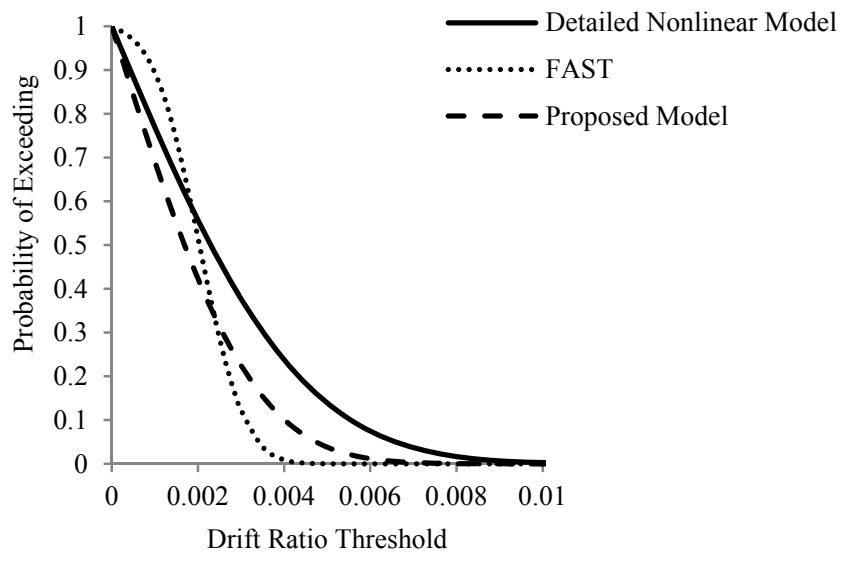

(b)

Fig 6-11. Probability of exceeding drift ratio thresholds versus threshold values for the example wind turbine at (a) rated wind speed and (b) cut-out wind speed 


\subsection{Summary and Conclusion}

With the assumption that the drift responses of wind turbines subject to wind and wave loads are Gaussian stationary processes, this section proposed a formulation for the estimation of probability and the expected time that the drift responses stay above a threshold. For the use of the proposed formulation, the mean and standard deviation of drifts are required to be estimated. One method to estimate the mean and standard deviation of drifts is to use the point estimation of those parameters at their mean values using the drifts obtained from simplified models in FAST. But since simplified models in FAST do not consider some of the important factors that affect the drift response of the support structure, such as soil-structure interaction, this section developed models to correct for the bias in the estimation obtained from FAST. In the developed models, correction terms were added to the mean and standard deviation of the drifts obtained from FAST to capture the bias inherent in those values due to simplification. The parameters in the correction terms were estimated using a regression analysis on the error values of the drift mean and standard deviation versus selected explanatory functions. Error values were defined as the difference between the responses of the detailed 3D nonlinear FE models in ABAQUS and the responses of the simplified models in FAST. Explanatory functions were selected among a set of candidates using a model selection process. Comparison between the estimation of expected time above a threshold using the developed method and the exact time above the same threshold using detailed 3D FE models showed that the developed method provides closer predictions to the measured values than FAST models. Probability of exceeding two specific drift 
thresholds at rated and cut-out wind speeds for a typical wind turbine were estimated using the developed method and results were compared to the corresponding results from detailed FE models and also FAST models. That comparison showed that the developed method is more successful than FAST in prediction of probability of exceeding drift thresholds. The merit of using the developed method is that it does not need to run time-consuming detailed 3D nonlinear FE models to capture the effects of some factors such as soil-structure interaction and can be easily used by engineers to estimate the expected time that the drift of a wind turbine exceeds a desired threshold. 


\section{CONCLUSIONS AND FUTURE WORK}

\subsection{Conclusions}

This dissertation investigated the structural reliability of offshore wind turbines. The focus of this work was on modern horizontal axis offshore wind turbines installed in water depth less than 30 meters, and supported by tubular steel tower and steel monopile foundation. Probabilistic models were developed to predict the deformation, shear and moment demands on wind turbine support structures, and calibrated using the information obtained from a database of virtual experiments. Virtual experiment data are generated by conducting detailed three dimensional (3D) nonlinear finite element (FE) analyses on a suite of typical offshore wind turbines. The FE models incorporated both aeroelastic interaction and the influence of soil-structure interaction in the dynamic response of the support structures. Separate probabilistic demand models were developed for three operational/load conditions including: (1) operating under day-today wind and wave loading; (2) operating throughout earthquake in presence of day-today loads; and (3) parked under extreme wind speeds and earthquake ground motions. The proposed approach gives special attention to the treatment of both aleatory and epistemic uncertainties in predicting the demands on the support structure of wind turbines. Furthermore, the probabilistic models provide unbiased predictions for the demands on the support structures, accounting for the inherent uncertainties, including the statistical uncertainty (associated with the finite sample size) and the modeling errors (associated with the selection of the variables in the models and the model form.) The 
developed demand models were used to assess the structural reliability of offshore wind turbines for specified performance levels. A probabilistic framework was then proposed for predicting the annual probability of structural failure of wind turbines given seismic and wind hazards for a specific location. Finally, the serviceability of wind turbines was explored in terms of wind turbines availability for power production, where the unavailability of wind turbine was investigated based on exceeding certain drift thresholds. The results of this study can be summarized as:

- Evaluating the accuracy of prevailing approaches for the analysis of laterally loaded mono-pile foundations revealed that depending on the pile diameter and soil type, using common simple methods such as $p-y$ method, and particularly modeling the pile employing one-dimensional beam-column elements may result in inaccurate responses. This is true in particular for the pile sizes typical of foundations of offshore wind turbines.

- Fragility curves assessed for a typical 5-MW offshore wind turbine using developed probabilistic demand models showed that the bending mode controls the probability of exceeding the yield and ultimate limit states, while the shear failure mode was found to provide negligible contributions to the fragility.

- The conditional probabilities of exceeding three specified performance levels (serviceability, yield, and ultimate) were found to increase with the average wind speed up to the rated wind speed. Upon reaching the rated wind speed, a control system is activated to limit the aerodynamic forces on the blades of the wind turbine and keep the power generated constant by changing the blade pitch angle. 
The activation of the control system was found to reduce the values of the fragilities.

- For the example wind turbine operating under day-to-day loads, the fragility estimates showed that wave loading does not noticeably affect the probability of failure, especially for large wind speeds.

- The fragility estimates for the wind turbine of interest operating throughout the earthquake indicated that wind speeds within the wind turbines' operational range do not noticeably affect the probability of failure in case of a seismic excitation, especially for large earthquakes.

- Annual probability of failure assessed for two identical 5-MW offshore wind turbines installed in two locations: Site I to be prone to hurricane; and Site II to be a high seismic region indicated that a wind turbine installed in a seismic region like California is more vulnerable to structural failure.

- With a high probability density of wind speeds close to rated wind velocity in Site II (off the California's Coast) and given the wind turbines operating at their maximum rate of power production at the rated wind speed, the California's Coast happens to be a great location candidate for an offshore wind farm with the superior wind resources for the power production.

\subsection{Unique Contributions}

This dissertation provided for the first time a probabilistic framework to investigate the structural reliability of offshore wind turbines subject to multiple hazards. The proposed 
approach can assist the wind industry decision makers choosing optimum design and location for future wind energy projects. The random vibration based framework developed to explore the performance of wind turbines can assist the wind industry to predict the availability for power production and accordingly, modify the structural design such that the expected time of staying above a drift threshold is limited to an acceptable level for the unavailability of power production.

\subsection{Future Work}

Some suggestions for future work based on the progress of the research here include:

- Using the developed probabilistic framework to assess the performance of wind farms. In this case, the developed demand models in this dissertation can be used for each individual wind turbine and then the probability of failure for the wind farm can be estimated using system reliability methods that include spatial correlation factors.

- Updating the developed probabilistic demand models using additional data to be generated considering other types of hazards such as high waves, storm surge, tornadoes, typhoons and tsunamis. Such models can provide more comprehensive predictions for multi-hazard reliability of wind turbines.

- Developing same probabilistic models for offshore wind turbines supported with other types of foundations such as tripods and floating foundations, as the wind industry is extending the wind farm projects to deeper waters because of the superior wind resources that are available farther from the coast. 


\section{REFERENCES}

ABAQUS (2007). Version 6.7-2, Hibbitt, Karlsson and Sorensen, Inc.

Agarwal, P., and Manuel, L. (2008). "Extreme Loads for an Offshore Wind Turbine Using Statistical Extrapolation from Limited Field Data." Wind Energy, 11(6), 673684.

Ang, A. H. S., and Tang, W. H. (2007). Probability concepts in engineering: Emphasis on applications to civil and environmental engineering. $2^{\text {th }}$ edition, John Wiley \&Sons, Inc: New York.

Arias, A. (1970). "A measure of earthquake intensity." Seismic design for nuclear power plants, R. J. Hansen, ed., MIT Press, Cambridge, MA, 438-483.

Baranov, V. A. (1967). "On the calculation of excited vibrations of an embedded foundation." (in Russian) Voprosy Dynamiki Prochnocti, 14, Polytechnic Institute of Riga, 195-209.

Bazeos, N., Hatzigeorgiou, G., Hondros, I., Karamaneas, H., Karabalis, D., et al. (2002). "Static, seismic and stability analyses of a prototype wind turbine steel tower." Engineering Structures, 24(8), 1015-1025.

Bisadi, V., Gardoni, P., and Head, M. (2012). "Probabilistic demand models and fragility estimates for bridges elevated with steel pedestals." Journal of Structural Engineering, In Press.

Blaney, G. W., Kausel, E., and Roesset, J. M. (1976). "Dynamic stiffness of piles." Proc., $2^{\text {nd }}$ Int. Conf. on Numerical Methods in Geomechanics, Blacksburg, VA, 10011012.

Bonnett, D. (2005). "Wind Turbine Foundations Loading, Dynamics and Design." Structural Engineer, 83(3), 41-45. 
Bossanyi, E. A. (2000). Bladed for windows user manual. Garrad Hassan and Partners, Bristol, UK.

Box, G. E. P., and Tiao, G. C. (1992). Baysian inference in statistical analysis, Wiley, New York.

Briaud, J. L. (1997). "Simple approach for lateral loads on piles." J. .Geotech. Engrg. Div., ASCE, 123(10), 958-964.

Briaud, J. L. (1992). The pressuremeter. A. A. Balkema, Rotterdam, The Netherlands.

Briaud, J. L., Smith, T. D., and Tucker, L. M. (1985). "A pressuremeter method for laterally loaded piles." Proc., $11^{\text {th }}$ Int. Conf. on Soil Mech. and Found. Engrg., 3, A. A. Balkema, Rotterdam, The Netherlands, 1353-1356.

Bush, B., and Manuel, L. (2009). "Foundation Models for Offshore Wind Turbines." 47th AIAA Aerospace Sciences Meeting Including the New Horizons Forum and Aerospace Exposition, January 5-8, Orlando, FL.

Burnham, K.P., and Anderson, D.R. (2002). Model selection and multimodel inference: a practical information-theoretic approach, $2^{\text {nd }}$ ed., Springer, New York.

CEN (2004). EN 10025. Hot rolled products of structural steels, Brussels, Belgium.

Chen, J.J., Zeng, Y.G., and Sun, H.A. (1996). "Dynamic reliability analysis of antenna reflector accuracy under wind excitation." Computers and Structures, 59(5), 819822.

Chioui, J. S., and Chenu, C. H. (2007). "Exact equivalent model for a laterallyloaded linear pile-soil system." Soils and Foundations, 47(6), 1053-1061.

Dean, R. G., and Dalrymple, R. A. (1991). Water wave mechanics for engineers and scientists. World Scientific, Singapore. 
Der Kiureghian, A., and Ke, J. B. (1985). "Finite-element based reliability analysis of frame structures." Proceedings of 4th International Conference on Structural Safety and Reliability (ICOSSAR), May 27-29, Kobe, Japan, 1, 395-404.

Det Norske Veritas (DNV). (2007). Design of offshore wind turbine structures. DNVOS-J10. Det Norske Veritas, Denmark.

Dewaikar, D. M., Salimath, R. S., and Sawant, V. A. (2009). "A modified P-Y curve for the analysis of a laterally loaded pile in stiff clay." Australian Geomechanics Journal, 44(3), 91-100.

DNV/Risø. (2002). Guidelines for design of wind turbines. $2^{\text {nd }}$ Edition, Det Norske Veritas and Wind Energy Department, Risø National Laboratory, Denmark.

Dueñas-Osorio, L., and Basu, B. (2008). "Unavailability of wind turbines due to windinduced accelerations." Engineering Structures, 30(4), 885-893.

Fink, D. (2005). "Small Wind Turbine Basics." Energy Self Sufficiency Newsletter, Aug. 2005, 17-23. 〈http://www.otherpower.com/windbasics2.html〉, Accessed in Sep. 2012.

Flowers, L. (2012). Wind energy update. National Renewable Energy Laboratory (NREL), 〈http://www.windpoweringamerica.gov/pdfs/wpa/wpa_update.pdf〉, Accessed in Sep. 2012.

Frankel, A. D., Peterson, M. D., Mueller C. S., Haller, K. M., Wheeler, R. L., et al. (2002). "Documentation for the 2002 update of the national seismic hazard maps." U.S. Geological Survey Open-File Report 02-20, USGS, Denver, CO.

Gardoni, P., Der Kiureghian, A., and Mosalam, K. M. (2002). "Probabilistic capacity models and fragility estimates for reinforced concrete columns based on experimental observations." J. Eng. Mech., 128(10), 1024-1038. 
Gardoni, P., Mosalam, K., and Der Kiureghian, M. A. (2003). "Probabilistic seismic demand models and fragility estimates for RC bridges." J. Earthquake Eng., 7(Sp. Issue 1), 79-106.

Gsänger, S., and Pitteloud, J. D. (2012). World wind energy report 2011. World Wind Energy Association (WWEA), 〈http://www.wwindea.org/webimages/

WorldWindEnergyReport2011.pdf), Accessed in May 2012.

Hau, E. (2006). Wind turbines: fundamentals, technologies, application, economics. $2^{\text {nd }}$ Edition, Springer, Berlin, Germany.

Hohenbichler, M., and Rackwitz, R. (1983). "First-order concepts in system reliability." Structural Safety, 1(3), 177-188.

Hurvich, C. M., and Tsai, C. (1989). "Regression and time series model selection in small samples." Biometrika, 76(2), 297-307.

IEC 61400-1. (2005). Wind turbines - Part 1: Design requirements. $3^{\text {rd }}$ Edition, Intl. Electrotechnical Commission, IEC.

Ishihara, T., and Sarwar, M. W. (2008). "Numerical and theoretical study on seismic response of wind turbines." Proceedings of European Wind Energy Conference (EWEC), 31 March-3April, Brussels, Belgium.

Jonkman , B. J. (2009). "TurbSim user's guide." Tech. Rep. NREL/TP-500-46198, National Renewable Energy Laboratory, Golden, CO.

Jonkman, J. M. (2007). "Dynamics Modeling and Loads Analysis of an Offshore Floating Wind Turbine." Tech. Rep. NREL/TP-500-41958.: National Renewable Energy Laboratory, Golden, CO.

Jonkman, J. M., and Buhl Jr., M. L. (2005). "FAST user's guide." Tech. Rep. NREL/EL500-38230, National Renewable Energy Laboratory, Golden, CO. 
Jonkman. J., Butterfield, S., Musial, W., and Scott, G. (2009). "Definition of a 5-MW reference wind turbine for offshore system development." Technical Report NREL/TP-500-38060, National Renewable Energy Laboratory, Golden, CO.

Kaimal, J. C., Wyngaard, J. C., Izumi, Y., and Cote, O. R. (1972). "Spectral characteristics of surface-layer turbulence." Quarterly Journal of the Royal Meteorological Society, 98, 563-589.

Kausel, E. (1974). "Forced vibrations of circular foundations on layered media." Research Report R 74-11, Civil Engineering Department, MIT, MA.

Kim, Y., and Jeong, S. (2011). "Analysis of soil resistance on laterally loaded piles based on 3D soil-pile interaction." Computers and Geotechnics, 38(2), 248-257.

Kolmogorov, A. (1933). "Sulla determinazione empirica di una legge di distribuzione." G. Inst. Ital. Attuari, 4, 83.

Krawinkler, H., Medina, R., and Alavi, B. (2003). "Seismic drift and ductility demands and their dependence on ground motions." Engineering Structures, 25(5), 637-653.

Laino, D. J., and Hansen, A. C. (2001). "User's guide to the computer software routines AeroDyn interface for ADAMS ${ }^{\circledR}$." Windward Engineering LLC, prepared for the National Renewable Energy Laboratory under Subcontract No. TCX-9-29209-01, Salt Lake City, UT.

Laino, D. J., and Hansen, A. C. (2002). "User's guide to the wind turbine aerodynamics computer software AeoDyn." Technical Report, Salt Lake City, UT.

Lavassas, I., Nikolaidis, G., Zervas, P., Efthimiou, E., Doudoumis, I. N., et al. (2003). "Analysis and design of the prototype of a steel 1-mw wind turbine tower." Engineering Structures, 25(8), 1097-106. 
Lee, K. H., and Rosowsky, D. V. (2007). "Synthetic hurricane wind speed records: development of a database for hazard analysis and risk studies." ASCE Natural Hazards Review, 8(2), 23-34.

Lutes, L.D., and Sarkani, S. (2003). Random Vibrations: Analysis of structural and mechanical systems, Elsevier Butterworth-Heinemann, MA.

Madsen, P.H., Pierce, K., and Buhl, M. (1999). "Predicting ultimate loads for wind turbine design." Proceedings of AIAA/ASME Wind Energy Symposium, January 1114, AIAA, Reno, NV, 355-364.

Mallows, C. L. (1973). "Some comments on CP.” Technometrics, 15 (4), 661-75.

Manuel, L., Veers, P. S., and Winterstein, S. R. (2001). "Parametric models for estimating wind turbine fatigue loads for design." ASME Journal of Solar Energy Engineering, 123(4), 346-355.

Matlock, H. (1970). "Correlations for design of laterally loaded piles in soft clay." Proc., $2^{\text {nd }}$ Annu. Offshore Technol. Conf., Paper 1204, 1, 577-588.

McKay, M. D., Conover, W. J., and Beckman, R. J. (1979). "A comparison of three methods for selecting values of input variables in the analysis of output from a computer code." Technometrics, 22(2), 239-45.

Morgan, E. C., Lackner, M., Vogel, R. M., and Baise, L. G. (2011). "Probability distribution for offshore wind speeds." Energy Conversion and Management, 52, 1526.

Murtagh, P.J., Basu, B., and Broderick, B.M. (2005). "Along-wind response of a wind turbine tower with blade coupling subjected to rotationally sampled wind loading." Engineering Structures, 27(8), 1209-1219. 
Musial, W., and Butterfield, S. (2004). "Future for offshore wind energy in the united states." Proceedings of the Energy Ocean Conference, 28-29 June, Palm Beach, Florida; Conference Paper NREL/CP-500-36313, NREL (2004), Golden, CO.

NOAA's National Data Buoy Center (NDBC). (2009). <http://www.ndbc.noaa.gov/>, Accessed in July 2009.

Novak, M. (1975). "Dynamic stiffness and damping of piles." J. Can. Geotech. Engrg., NRC of Canada, 11(5), 574-698.

Pacific Earthquake Engineering Research Center. (1999). "Pacific Earthquake Engineering Research Center: NGA Database." 〈http://peer.berkeley.edu/nga〉, Accessed in Sep. 2011.

Prowell, I., and Veers, P., (2009). "Assessment of wind turbine seismic risk: Existing literature and simple study of tower moment demand." SAND2009-1100, Sandia National Laboratories, Albuquerque, NM.

Prowell, I., Veletsos, M., and Elgamal, A. (2008). "Full scale testing for investigation of wind turbine seismic response." Proceedings of the 7th World Wind Energy Conference, June 24-26, Kingston, Ontario, Canada.

Prowell, I., Elgamal, A., and Jonkman, J. (2009). "FAST simulation of wind turbine seismic response." Proceedings of the Asian-Paciific Network for Earthquake Engineering Research (ANCER) Workshop, 13-14 August, Urbana-Champaign, Illinois; Conference Paper NREL/CP-500-46225, NREL (2010), Golden, CO.

Ramamoorthy, K. S., Gardoni, P., and Bracci, M. J. (2006). "Probabilistic demand models and fragility curves for reinforced concrete frames." Journal of Structural Engineering, 132(10):1563-1572.

Reese, L. C., and Welch, R. C. (1975). "Lateral loading of deep foundations in stiff clay." J. Geotech. Engrg. Div., ASCE, 101(7), 633-649. 
Reese, L. C., and Wang, S. T. (2008). "Design of foundations for a wind turbine employing modern principles." J.E. Laier, D.K. Crapps and M.H. Hussein (eds), From Research to Practice in Geotechnical Engineering, ASCE Geotech. Spec. Publ., No. 180, 351-65.

Reese, L. C., Cox, W. R., and Koop, F. D. (1974). "Analysis of laterally loaded piles in sand." Proc., $6^{\text {th }}$ Annu. Offshore Technol. Conf., Paper 2080, 2, 473-483.

Reese, L. C., Cox, W. R., and Koop, F. D. (1975). "Field testing and analysis of laterally loaded piles in stiff clay." Proc., $7^{\text {th }}$ Annu. Offshore Technol. Conf., Paper 2312, 2 , 671-690.

Ren, N., Li, Y., and Ou, J. (2010). "The motion performance of two offshore wind turbine floating platforms with combined tension leg-mooring line system." Proceedings of the Ninth ISOPE Pacific/Asia Offshore Mechanics Symposium, November 14-17, Busan, Korea.

Rezaeian, S., and Der Kiureghian, A. (2010). "Stochastic modeling and simulation of ground motions for performance-based earthquake engineering." PEER 2010/02, University of California, Berkeley, CA.

Sanchez Salinero, I. (1982). "Static and dynamic stiffnesses of single piles." Geotechnical Engineering Report GR82-31, The University of Texas at Austin.

Sanjaya Kumar, V., Sharma, K. G., and Varadarajan, A. (2007). "Behaviour of a laterally loaded pile." Proc. 10th International Symposium on Numerical Models in Geomechanics NUMOG 10, 447-452.

Sawyer, S., and Rave, K. (2012). Annual market update2011. Global Wind Energy Council (GWEC), March 2012. Available at: http://www.gwec.net/fileadmin/ documents/NewsDocuments/Annual report_2011_lowres.pdf. 
Schwarz, G. E. (1978). "Estimating the dimension of a model." Annals of Statistics, 6(2), $461-464$.

Schwartz, M., Heimiller, D., Haymes, S., and Musial, W. (2010). "Assessment of offshore wind energy resources for the United States." Tech. Rep. NREL/TP-50045889, NREL, Golden, CO.

Shome, N., and Cornell, C. A. (1999). "Probabilistic seismic demand analysis of nonlinear structures." Reliability of Marine Structures Rep. No. RMS-35, Dept. of Civil and Envir. Engineering, Stanford Univ., Palo Alto, CA.

Simpson, T. W., Lin, D. K. J., and Chen, W. (2001). "Sampling strategies for computer experiments: design and analysis." Intl. J. Reliability and Application, 2(3), 209-40.

Suleiman, M., Raich, A., Polson, T. W., Kingston, and W. J., Roth, M. (2010). "Measured soil-pile interaction pressures for small-diameter laterally loaded pile in loose sand." Proc., GeoFlorida 2010 on Advances in Analysis, Modeling and Design, Geotechnical Special Publication, Paper 199, 1498-1506.

Suzuki, H., Kitahara, Y., and Fukumoto, Y. (2011). "Progressive Drift of Moored Floating Wind Turbines in a Wind Farm - Improved Mooring Capacity Model." Proceedings of the ASME 2011 30th International Conference on Ocean, Offshore and Arctic Engineering, OMAE2011, June 19-24, Rotterdam, the Netherlands.

Tavner, P.J., Xiang, J., and Spinato, F. (2007). "Reliability Analysis for Wind Turbines." Wind Energy, 10(1), 1-18.

Theil, H. (1961). Economic forcasts and policy. North-Holland Pub. Co., Amsterdam, The Netherlands.

Vanmarcke, E. (2010). Random fields, World Scientific Publishing Co. Pte. Ltd., Singapore. 
Walford, C. A. (2006). "Wind turbine reliability: understanding and minimizing wind turbine operation and maintenance costs." Rep. No. SAND2006-1100, Sandia National Laboratories, Albuquerque, NM and Livermore, CA.

Wang, Y. (2010). "Studies on hazard characterization for performance based structural design." Ph.D. Dissertation, Department of Civil Engineering, Texas A\&M University, College Station, TX.

Witcher, D. (2005). "Seismic analysis of wind turbines in the time domain." Wind Energy, $8(1), 81-91$. 